\title{
Gravitational Cardy limit and AdS black hole entropy
}

\author{
Marina David, ${ }^{a}$ Jun Nian ${ }^{a}$ and Leopoldo A. Pando Zayas ${ }^{a, b}$ \\ ${ }^{a}$ Leinweber Center for Theoretical Physics, University of Michigan, \\ Ann Arbor, MI 48109, U.S.A. \\ ${ }^{b}$ The Abdus Salam International Centre for Theoretical Physics, \\ 34014 Trieste, Italy \\ E-mail: mmdavid@umich.edu, nian@umich.edu, lpandoz@umich.edu
}

\begin{abstract}
We explore the gravitational implementation of the field theory Cardy-like limit recently used in the successful microstate countings of AdS black hole entropy in various dimensions. On the field theory side, the Cardy-like limit focuses on a particular scaling of conserved electric charges and angular momenta and we first translate this scaling to the gravitational side by a limiting procedure on the black hole parameters. We note that the scaling naturally accompanies a near-horizon region for which these black hole solutions are greatly simplified. Applying the Kerr/CFT correspondence to the near-horizon region, we precisely reproduce the Bekenstein-Hawking entropy of asymptotically $\mathrm{AdS}_{4,5,6,7}$ BPS black holes. Our results explicitly provide a microscopic and universal low energy description for AdS black holes across various dimensions.
\end{abstract}

KeYwords: AdS-CFT Correspondence, Black Holes in String Theory, Black Holes

ArXiv EPrint: 2005.10251 


\section{Contents}

1 Introduction 1

2 Asymptotically AdS $_{5}$ black holes $\quad 4$

$2.1 \mathrm{AdS}_{5}$ black hole solution 4

2.2 Gravitational Cardy limit $\quad 7$

2.3 Black hole solution in the near-horizon + gravitational Cardy limit 8

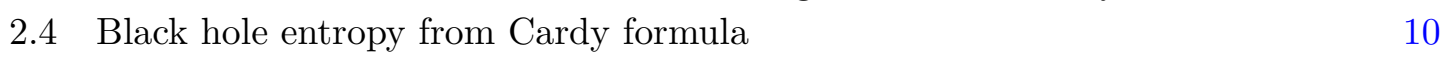

$\begin{array}{ll}2.5 & \text { Comparison with results from boundary CFT } \\ \end{array}$

3 Asymptotically AdS $_{4}$ black holes $\quad 14$

$\begin{array}{lll}3.1 & \mathrm{AdS}_{4} \text { black hole solution } & 15\end{array}$

$\begin{array}{ll}3.2 \text { Gravitational Cardy limit } & 16\end{array}$

3.3 Black hole solution in the near-horizon + gravitational Cardy limit $\quad 17$

$\begin{array}{ll}3.4 & \text { Black hole entropy from Cardy formula } \\ & 18\end{array}$

$\begin{array}{ll}\text { 3.5 Comparison with results from boundary CFT } & 19\end{array}$

4 Asymptotically AdS $_{7}$ black holes $\quad 20$

$\begin{array}{lll}4.1 \text { A special case } & 20\end{array}$

$\begin{array}{lll}\text { 4.1.1 } & \mathrm{AdS}_{7} \text { black hole solution } & 20\end{array}$

4.1.2 Gravitational Cardy limit 23

4.1.3 Black hole solution in the near-horizon + gravitational Cardy limit 24

4.1.4 Black hole entropy from Cardy formula 24

$\begin{array}{ll}\text { 4.1.5 Comparison with results from boundary CFT } & 25\end{array}$

$\begin{array}{lll}4.2 & \text { More general case } & 26\end{array}$

$\begin{array}{lll}\text { 4.2.1 } & \mathrm{AdS}_{7} \text { black hole solution } & 26\end{array}$

$\begin{array}{lll}\text { 4.2.2 } & \text { Gravitational Cardy limit } & 29\end{array}$

4.2.3 Black hole solution in the near-horizon limit 30

4.2.4 Black hole entropy from Cardy formula 32

4.2.5 Comparison with results from boundary CFT 32

5 Asymptotically AdS $_{6}$ black holes $\quad 32$

5.1 $\mathrm{AdS}_{6}$ black hole solution $\quad 33$

5.2 Gravitational Cardy limit $\quad 35$

5.3 Black hole solution in the near-horizon + gravitational Cardy limit 36

$\begin{array}{ll}5.4 & \text { Black hole entropy from Cardy formula }\end{array}$

$\begin{array}{ll}\text { 5.5 Comparison with results from boundary CFT } & 38\end{array}$

$\begin{array}{lll}6 & \text { Discussion } & 38\end{array}$ 
A.1 $A d S_{5} \quad 41$

$\begin{array}{lll}\text { A.2 } & A d S_{4} & 43\end{array}$

$\begin{array}{lll}\text { A.3 } & A d S_{7} & 45\end{array}$

$\begin{array}{lll}\text { A.4 } & A d S_{6} & 47\end{array}$

\section{Introduction}

The AdS/CFT correspondence [1] posits an equivalence between string theory in certain asymptotically AdS spacetimes and the corresponding dual conformal field theories. This paradigm provides implicit resolutions to many of the puzzles presented by gravity as it can now be reformulated in terms of a unitary field theory. A necessary first step for this program involves the microscopic counting of the degrees of freedom responsible for the Bekenstein-Hawking entropy of asymptotically AdS black holes. In the context of string theory on $\mathrm{AdS}_{5} \times S^{5}$, a powerful early attempt to the microscopic computation of the black hole entropy was put forward in [2]; such an attempt has been recently improved in [3-5] to provide a precise matching between the Bekenstein-Hawking entropy of the black hole and the microstate counting in the dual $\mathcal{N}=4$ SYM theory. The key idea in these recent works has been to consider complex backgrounds or complex chemical potentials. This approach has now been generalized to include the microscopic counting of the entropy of rotating, electrically charged asymptotically AdS black holes in various dimensions [6-14]. These recent works, together with previous progress on magnetically charged asymptotically AdS black holes $[15,16]$, provide a fairly complete and novel approach to microstate counting for asymptotically AdS black holes and mark the beginning of a new era in explorations of quantum gravity.

A so-called Cardy-like limit has played a prominent role among the recent literature on microstate counting dual to the entropy of rotating electrically charged asymptotically AdS black holes [4, 6, 8-10]; the limit is loosely defined as

$$
\left|\omega_{i}\right| \ll 1
$$

i.e., the angular velocities are very small compared to other parameters on the field theory side. The Cardy-like limit above has the advantage of greatly simplifying the analysis of the effective large $-N$ matrix model obtained from the superconformal index or the partition function of the boundary conformal field theory.

Let us discuss this limit by focusing on the states or operators it singles out. One interpretation of the Cardy-like limit follows from selecting a particular sector in the dual field theory. More precisely, we consider the entropy function given schematically as $S=$ $I+\sum_{i} \omega_{i} J_{i}+\sum_{I} \Delta_{I} Q_{I}-\Lambda\left(\sum_{I} \Delta_{I}-\sum_{i} \omega_{i}-2 \pi i\right)$ in terms of conserved charges, chemical potentials and a Lagrange multiplier, $\Lambda$, and search for a self-consistent scaling of the form $\omega_{i} \sim \epsilon$ and $\Delta_{I} \sim \mathcal{O}(1)$. Demanding that all terms contribute at the same order in $\epsilon$ leads to the following scalings of quantum numbers in table 1 that describes the singled-out sector. 


\begin{tabular}{|c|c|c|c|c|c|}
\hline Dimension of CFT & $\omega$ & $\Delta$ & $J$ & $Q$ & Entropy Function \\
\hline$d=3$ & $\epsilon$ & 1 & $1 / \epsilon^{2}$ & $1 / \epsilon$ & $1 / \epsilon$ \\
\hline$d=4$ & $\epsilon$ & 1 & $1 / \epsilon^{3}$ & $1 / \epsilon^{2}$ & $1 / \epsilon^{2}$ \\
\hline$d=5$ & $\epsilon$ & 1 & $1 / \epsilon^{3}$ & $1 / \epsilon^{2}$ & $1 / \epsilon^{2}$ \\
\hline$d=6$ & $\epsilon$ & 1 & $1 / \epsilon^{4}$ & $1 / \epsilon^{3}$ & $1 / \epsilon^{3}$ \\
\hline
\end{tabular}

Table 1. Scaling of conserved quantum numbers in various field theory dimensions.

The self-consistency of these scalings is checked a posteriori. Since the charges (angular momenta and electric charges) are large, the intuition for a semiclassical regime is justified. It is precisely in this regime that the Legendre transform to obtain the entropy as the extremization of the entropy function is fully justified. ${ }^{1}$

In this paper we study the Cardy-like limit on the gravity side, which we refer to as the gravitational Cardy limit. To define this limit, we start with the field theory Cardylike limit (1.1) and find its counterpart on the gravity side using some relations between field theory and gravity parameters. More explicitly, the gravitational Cardy limit has specific expressions for various examples considered in this paper given by (2.18), (3.18), (4.18), (4.52) and (5.16), which impose some special limits on the parameters of the corresponding black hole solutions. For all the asymptotically $\mathrm{AdS}_{4,5,6,7}$ solutions studied in this paper, the gravitational Cardy limit can be universally written as

$$
\left|a_{i} g\right| \rightarrow 1
$$

where $a_{i}$ roughly characterize angular momenta in units of the inverse radius of AdS, $g$. It turns out that when combined with a particular near-horizon limit for rotating asymptotically AdS BPS black holes, the gravitational Cardy limit provides further insight by yielding a near-horizon $\mathrm{AdS}_{3}$ geometry, independent of the dimension of the black hole. Hence, the black hole entropy can be obtained from this near-horizon $\mathrm{AdS}_{3}$ a la the Kerr/CFT correspondence.

More specifically, the asymptotically flat extremal black hole entropy can be obtained using the Kerr/CFT correspondence near the horizon region [18], and this approach has been generalized to asymptotically AdS extremal black holes $[19,20]$. The main idea is to apply the limit introduced in [19-21] to probe a particular near-horizon region of extremal black holes. In this limit the near-horizon geometry is simplified to be some circles fibered over $\mathrm{AdS}_{2}$, from which one can find a Virasoro algebra and, consequently, compute the corresponding central charge and Frolov-Thorne temperature. Using the Cardy formula, the black hole entropy can be successfully reproduced for extremal black holes. The conceptual advantage of this approach is that it microscopically explains the black hole entropy based on general near-horizon symmetries and does not require intimate knowledge of the full higher-dimensional dual CFT. The situation is akin to microscopically computing the entropy of the AdS black holes without full knowledge of the UV complete theory, which in this case is the higher-dimensional boundary CFT. We thus present

\footnotetext{
${ }^{1}$ Our intuition closely follows the BMN paradigm where a closed sector of large R-charge operators of a given scaling is singled out [17].
} 


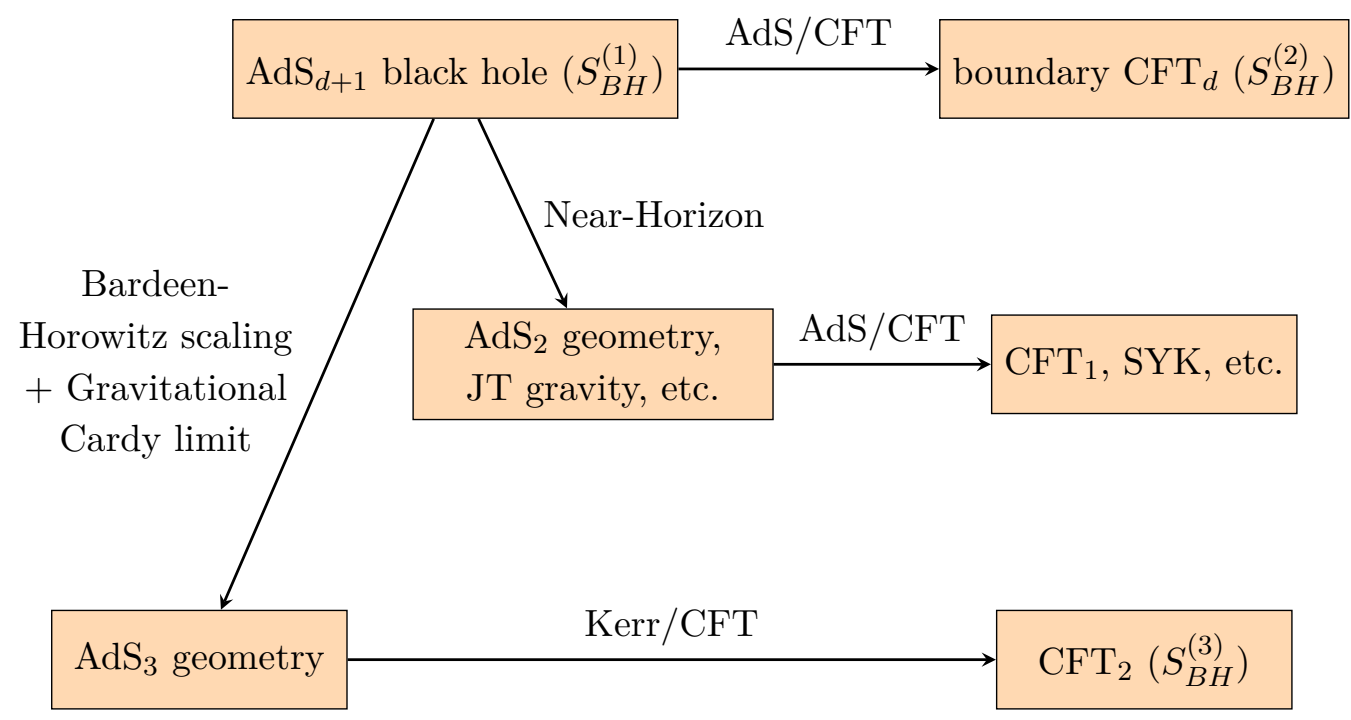

Figure 1. The Gravitational Cardy limit plays a role in the broader picture of the study of AdS black holes, giving us a near-horizon $\mathrm{AdS}_{3}$ geometry, similar to that of the extremal vanishing horizon limit. The entropy can be computed in three separate regimes $\left(S_{B H}^{(i)}\right)$, and are all found to be equal.

steps toward certain universality of asymptotically AdS black holes. This is similar to Strominger's discussion of the entropy of asymptotically flat black holes, using only the near-horizon geometry [22] without recourse to the full string theory description originally used in [23].

In this paper, we apply the same near-horizon limit introduced in [19-21] to the BPS, i.e., to supersymmetric and extremal, asymptotically AdS black holes in various dimensions. As a new ingredient, we take the gravitational Cardy limit, and the near-horizon geometry will be further reduced to only one $\mathrm{U}(1)$ fibered over $\mathrm{AdS}_{2}$, or equivalently, an $\mathrm{AdS}_{3}$ geometry. Hence, the gravitational Cardy limit provides the minimal amount of information for computing the black hole entropy using the Kerr/CFT correspondence. We will demonstrate this statement using several examples of asymptotically AdS black holes in various dimensions.

A pictorial way to summarize the situation is presented in figure 1 where we indicate how to reproduce the Bekenstein-Hawking entropy of asymptotically $\mathrm{AdS}_{d+1}$ BPS black holes from two points of view: (i) the boundary $\mathrm{CFT}_{d}$ and (ii) the near-horizon Virasoro algebra arising as the asymptotic symmetry algebra.

The near-horizon Virasoro-based results are simpler. The most important point, however, is that our explicit computations demonstrate the existence of certain universality for the class of rotating electrically charged asymptotically AdS black holes in dimensions 4, 5, 6 and 7. This universality manifests itself as an underlying $\mathrm{AdS}_{3} / \mathrm{CFT}_{2}$ correspondence living in the near-horizon region.

To further gain intuition into the gravitational Cardy limit we discuss in this paper, it is instructive to compare our approach with a somewhat related Extremal Vanishing 
Horizon (EVH) limit discussed in [24] (see also [25-28]). For asymptotically $\operatorname{AdS}_{d+1}$ BPS black holes, the authors of [24] related the boundary $\mathrm{CFT}_{d}$ and the corresponding nearhorizon computations in the EVH limit. In this case, there arises an effective $2 \mathrm{~d}$ CFT obtained in the near-horizon region. However, the (near-)EVH limit is strictly restricted to the (nearly) vanishing horizon case, i.e., $A_{\mathrm{BH}} \rightarrow 0, T \rightarrow 0, A_{\mathrm{BH}} / T \rightarrow$ constant. In contrast, the gravitational Cardy limit works for generic asymptotically AdS BPS black holes without restricting the horizon area. This is because the gravitational Cardy limit also rescales the conserved charges.

This paper is organized as follows. In section 2 through section 5 we discuss the asymptotically $\mathrm{AdS}_{5}, \mathrm{AdS}_{4}, \mathrm{AdS}_{7}$ and $\mathrm{AdS}_{6}$ cases, respectively. For each case, we first review the black hole solution, and then define the corresponding gravitational Cardy limit. After taking the gravitational Cardy limit under the Bardeen-Horowitz near-horizon scaling, we can see a structure of Virasoro algebra and compute the black hole entropy using the microscopic Cardy formula, which matches the results from the boundary $\mathrm{CFT}_{d}$ and the Bekenstein-Hawking formula for $\mathrm{AdS}_{d+1}$. Some possible extensions for future research are discussed in section 6 . In appendix A, we present some details of verifying the equations of motion for the near-horizon geometries.

\section{Asymptotically $\mathrm{AdS}_{5}$ black holes}

In this section, we consider the asymptotically $\mathrm{AdS}_{5}$ black holes and the corresponding gravitational Cardy limit. We will demonstrate that the black hole entropy can be computed in various ways as shown in figure 1, and that the other thermodynamic quantities scale in the gravitational Cardy limit precisely as in the field theory approach following table 1.

\section{1 $\quad \mathrm{AdS}_{5}$ black hole solution}

In this subsection, we first review the non-extremal asymptotically $\mathrm{AdS}_{5}$ black hole solution found in [29] with degenerate electric charges $Q_{1}=Q_{2}=Q_{3}=Q$ and two angular momenta $J_{1,2}$, and then take the BPS limit to obtain its supersymmetric version.

The non-extremal asymptotically $\mathrm{AdS}_{5}$ black hole background was found in [29] as a solution to the equations of motion of the $5 \mathrm{~d}$ minimal gauged supergravity in the BoyerLindquist coordinates $x^{\mu}=(t, r, \theta, \phi, \psi)$. The metric and the gauge field of the black hole solution are given by

$$
\begin{aligned}
d s^{2}= & -\frac{\Delta_{\theta}\left[\left(1+g^{2} r^{2}\right) \rho^{2} d t+2 q \nu\right] d t}{\Xi_{a} \Xi_{b} \rho^{2}}+\frac{2 q \nu \omega}{\rho^{2}}+\frac{f}{\rho^{4}}\left(\frac{\Delta_{\theta} d t}{\Xi_{a} \Xi_{b}}-\omega\right)^{2}+\frac{\rho^{2} d r^{2}}{\Delta_{r}}+\frac{\rho^{2} d \theta^{2}}{\Delta_{\theta}} \\
& +\frac{r^{2}+a^{2}}{\Xi_{a}} \sin ^{2} \theta d \phi^{2}+\frac{r^{2}+b^{2}}{\Xi_{b}} \cos ^{2} \theta d \psi^{2} \\
A= & \frac{\sqrt{3} q}{\rho^{2}}\left(\frac{\Delta_{\theta} d t}{\Xi_{a} \Xi_{b}}-\omega\right)+\alpha_{5} d t
\end{aligned}
$$


where

$$
\begin{aligned}
\nu & \equiv b \sin ^{2} \theta d \phi+a \cos ^{2} \theta d \psi \\
\omega & \equiv a \sin ^{2} \theta \frac{d \phi}{\Xi_{a}}+b \cos ^{2} \theta \frac{d \psi}{\Xi_{b}} \\
\Delta_{\theta} & \equiv 1-a^{2} g^{2} \cos ^{2} \theta-b^{2} g^{2} \sin ^{2} \theta \\
\Delta_{r} & \equiv \frac{\left(r^{2}+a^{2}\right)\left(r^{2}+b^{2}\right)\left(1+g^{2} r^{2}\right)+q^{2}+2 a b q}{r^{2}}-2 m \\
\rho^{2} & \equiv r^{2}+a^{2} \cos ^{2} \theta+b^{2} \sin ^{2} \theta \\
\Xi_{a} & \equiv 1-a^{2} g^{2} \\
\Xi_{b} & \equiv 1-b^{2} g^{2} \\
f & \equiv 2 m \rho^{2}-q^{2}+2 a b q g^{2} \rho^{2}
\end{aligned}
$$

and $\alpha_{5} d t$ is a pure gauge term with $\alpha_{5}$ a constant. These black hole solutions are characterized by four independent parameters $(a, b, m, q)$. The thermodynamical quantities, including the mass $E$, the temperature $T$ and the entropy $S$, can all be expressed in terms of these independent parameters. The other physical quantities, such as the electric charge $Q$, the electric potential $\Delta$, the angular momenta $J_{1,2}$ and the angular velocities $\Omega_{1,2}$ can similarly be written in terms of the four independent parameters. For example, the gravitational angular velocities $\Omega_{1,2}$ and the temperature $T$ are given by

$$
\begin{aligned}
\Omega_{1} & =\frac{a\left(r_{+}^{2}+b^{2}\right)\left(1+g^{2} r_{+}^{2}\right)+b q}{\left(r_{+}^{2}+a^{2}\right)\left(r_{+}^{2}+b^{2}\right)+a b q}, \\
\Omega_{2} & =\frac{b\left(r_{+}^{2}+a^{2}\right)\left(1+g^{2} r_{+}^{2}\right)+a q}{\left(r_{+}^{2}+a^{2}\right)\left(r_{+}^{2}+b^{2}\right)+a b q}, \\
T & =\frac{r_{+}^{4}\left[1+g^{2}\left(2 r_{+}^{2}+a^{2}+b^{2}\right)\right]-(a b+q)^{2}}{2 \pi r_{+}\left[\left(r_{+}^{2}+a^{2}\right)\left(r_{+}^{2}+b^{2}\right)+a b q\right]},
\end{aligned}
$$

where $r_{+}$denotes the position of the outer horizon given by the largest root of $\Delta_{r}$ in (2.3).

As carefully discussed in [3], it is crucial to make the following important distinctions of these solutions, in the broader context when complex potentials are allowed. The extremal black hole solution is characterized by the appearance of a double root in $\Delta_{r}=0$, while the BPS black hole solution is obtained by solving the supersymmetry equations.

The BPS limit is a special limit in the parameter space, such that the backgrounds in this limit are both extremal and supersymmetric. For the class of $\mathrm{AdS}_{5}$ black hole solutions (2.1), the BPS limit corresponds to the following condition

$$
q=\frac{m}{1+a g+b g} .
$$

Moreover, to prevent unphysical naked closed timelike curves (CTC), it is shown in [29] that the BPS solutions should further satisfy the constraint

$$
m=\frac{1}{g}(a+b)(1+a g)(1+b g)(1+a g+b g) .
$$


Hence, in the BPS limit only two of the four parameters $(a, b, m, q)$ are independent, which can be chosen to be $(a, b)$. The special case $a=b$ corresponds to the supersymmetric $\mathrm{AdS}_{5}$ black hole solutions found by Gutowski and Reall [30]. In the BPS limit, the outer horizon $r_{+}$coincides with the inner horizon at $r_{0}$

$$
r_{0}^{2}=\frac{a+b+a b g}{g}
$$

and the black hole entropy $S_{*}$, the electric charge $Q_{*}$ and the angular momenta $J_{1,2}^{*}$ have the following expressions in terms of $(a, b)$

$$
\begin{aligned}
S_{*} & =\frac{\pi^{2}(a+b) \sqrt{a+b+a b g}}{2 g^{3 / 2}(1-a g)(1-b g)}, \\
Q_{*} & =\frac{\pi(a+b)}{4 g(1-a g)(1-b g)}, \\
J_{1}^{*} & =\frac{\pi(a+b)(2 a+b+a b g)}{4 g(1-a g)^{2}(1-b g)}, \\
J_{2}^{*} & =\frac{\pi(a+b)(a+2 b+a b g)}{4 g(1-a g)(1-b g)^{2}},
\end{aligned}
$$

where the entropy $S_{*}$ is computed from the Bekenstein-Hawking entropy formula

$$
S_{B H}=\frac{A}{4 G_{N}},
$$

a quarter of the horizon area in units of Planck length. Using the expressions (2.8), we can also rewrite the black hole entropy as a function of $Q$ and $J_{1,2}$

$$
S_{B H}=2 \pi \sqrt{\frac{3 Q^{2}}{g^{2}}-\frac{\pi}{4 g^{3}}\left(J_{1}+J_{2}\right)} .
$$

The AdS/CFT dictionary helps translate the parameters of the $\mathrm{AdS}_{5}$ black holes to quantities in $\mathcal{N}=4 \mathrm{SYM}$

$$
\frac{1}{2} N^{2}=\frac{\pi}{4 G_{N}} \ell_{5}^{3}
$$

with $\ell_{5}=g^{-1}$ denoting the $\mathrm{AdS}_{5}$ radius. We can rewrite the expression (2.10) of the $\mathrm{AdS}_{5}$ black hole entropy (in the unit $G=1$ )

$$
S_{B H}=2 \pi \sqrt{\frac{3 Q^{2}}{g^{2}}-\frac{N^{2}}{2}\left(J_{1}+J_{2}\right)} .
$$

This expression has recently been extracted directly from the boundary CFT in [3-5] with further clarifying field theory work presented in [31-40]. We show below that this boundary CFT result can also be obtained from a particular near-horizon Cardy formula. 


\subsection{Gravitational Cardy limit}

The Cardy-like limit for the $\mathcal{N}=4 \mathrm{SYM}$ index was defined in [4]. This limit has been discussed in the context of $\mathcal{N}=4 \mathrm{SYM}$ also in [31, 32, 39]. In the more general context of $\mathcal{N}=1$ superconformal theories, it has been discussed in [33-35]. A key ingredient in the limit is the regime

$$
\left|\omega_{i}\right| \ll 1, \quad \Delta_{I} \sim \mathcal{O}(1), \quad(i=1,2 ; I=1,2,3) .
$$

Using the relation found in $[3,38]$

$$
\operatorname{Re}\left(\omega_{i}\right)=\left.\frac{\partial \Omega_{i}}{\partial T}\right|_{T=0}, \quad \operatorname{Re}\left(\Delta_{I}\right)=\left.\frac{\partial \Phi_{I}}{\partial T}\right|_{T=0},
$$

we can express the Cardy-like limit (2.13) in terms of quantities in the dual gravity theory, such that

$$
\left|\left(\frac{\partial \Omega_{i}}{\partial T}\right)_{T=0}\right| \ll 1,\left.\quad \frac{\partial \Phi_{I}}{\partial T}\right|_{T=0} \sim \mathcal{O}(1),
$$

with $i=1,2$ and $I=1,2,3$. Using the expressions of the thermodynamic quantities (2.4), we obtain for the asymptotically $\mathrm{AdS}_{5}$ BPS black holes,

$$
\begin{aligned}
& \left.\frac{\partial \Omega_{1}}{\partial T}\right|_{\mathrm{BPS}}=\lim _{T \rightarrow 0} \frac{\Omega_{1}-\Omega_{1}^{*}}{T}=\frac{2 \pi(-1+a g)}{3 g} \sqrt{\frac{1+a g+b g}{a b}}, \\
& \left.\frac{\partial \Omega_{2}}{\partial T}\right|_{\mathrm{BPS}}=\lim _{T \rightarrow 0} \frac{\Omega_{2}-\Omega_{2}^{*}}{T}=\frac{2 \pi(-1+b g)}{3 g} \sqrt{\frac{1+a g+b g}{a b}},
\end{aligned}
$$

where $\Omega_{1,2}^{*}$ are the values of $\Omega_{1,2}$ in the BPS limit. From the expressions of $\left.\frac{\partial \Omega_{i}}{\partial T}\right|_{\text {BPS }}$ $(i=1,2)$, we conclude that for asymptotically $\mathrm{AdS}_{5}$ BPS black holes, the gravitational Cardy limit corresponds to the special limit of the parameters on the gravity side

$$
a \rightarrow \frac{1}{g}, \quad b \rightarrow \frac{1}{g}
$$

For later convenience, we parameterize $a$ and $b$ as

$$
a=\frac{1}{g}-\epsilon, \quad b=\frac{1}{g}-\epsilon .
$$

For this case, $\epsilon$ has the dimension of length. Taking the gravitational Cardy limit (2.18) for the parameters into account, the BPS thermodynamic quantities (2.8) become

$$
\begin{aligned}
S_{*} & =\frac{\sqrt{3} \pi^{2}}{g^{5} \epsilon^{2}}+\mathcal{O}\left(\epsilon^{-1}\right), \\
Q_{*} & =\frac{\pi}{2 g^{4} \epsilon^{2}}+\mathcal{O}\left(\epsilon^{-1}\right), \\
J_{1}^{*} & =\frac{2 \pi}{g^{6} \epsilon^{3}}+\mathcal{O}\left(\epsilon^{-2}\right), \\
J_{2}^{*} & =\frac{2 \pi}{g^{6} \epsilon^{3}}+\mathcal{O}\left(\epsilon^{-2}\right),
\end{aligned}
$$

which are precisely the scalings of the field theory results $[4,41]$. 


\subsection{Black hole solution in the near-horizon + gravitational Cardy limit}

In the previous subsection, we have obtained the gravitational Cardy limit for the parameters on the gravity side. In this subsection, we discuss how the near-horizon metric changes in this limit as well as clarify other ingredients.

The asymptotically $\mathrm{AdS}_{5}$ metric (2.1) can be written in the following equivalent form, which is more convenient for the discussions in this subsection,

$d s^{2}=-\frac{\Delta_{r} \Delta_{\theta} r^{2} \sin ^{2}(2 \theta)}{4 \Xi_{a}^{2} \Xi_{b}^{2} B_{\phi} B_{\psi}} d t^{2}+\rho^{2}\left(\frac{d r^{2}}{\Delta_{r}}+\frac{d \theta^{2}}{\Delta_{\theta}}\right)+B_{\psi}\left(d \psi+v_{1} d \phi+v_{2} d t\right)^{2}+B_{\phi}\left(d \phi+v_{3} d t\right)^{2}$,

where

$$
\begin{array}{rlrl}
B_{\phi} & \equiv \frac{g_{33} g_{44}-g_{34}^{2}}{g_{44}}, & B_{\psi} \equiv g_{44}, \\
v_{1} \equiv \frac{g_{34}}{g_{44}}, \quad v_{2} \equiv \frac{g_{04}}{g_{44}}, & v_{3} \equiv \frac{g_{04} g_{34}-g_{03} g_{44}}{g_{34}^{2}-g_{33} g_{44}},
\end{array}
$$

with the non-vanishing components of the metric (2.1) explicitly in the coordinates $(t, r, \theta, \varphi, \psi)$

$$
\begin{aligned}
& g_{00}=-\frac{\Delta_{\theta}\left(1+g^{2} r^{2}\right)}{\Xi_{a} \Xi_{b}}+\frac{\Delta_{\theta}^{2}\left(2 m \rho^{2}-q^{2}+2 a b q g^{2} \rho^{2}\right)}{\rho^{4} \Xi_{a}^{2} \Xi_{b}^{2}}, \\
& g_{03}=g_{30}=-\frac{\Delta_{\theta}\left[a\left(2 m \rho^{2}-q^{2}\right)+b q \rho^{2}\left(1+a^{2} g^{2}\right)\right] \sin ^{2} \theta}{\rho^{4} \Xi_{a}^{2} \Xi_{b}}, \\
& g_{04}=g_{40}=-\frac{\Delta_{\theta}\left[b\left(2 m \rho^{2}-q^{2}\right)+a q \rho^{2}\left(1+b^{2} g^{2}\right)\right] \cos ^{2} \theta}{\rho^{4} \Xi_{b}^{2} \Xi_{a}}, \\
& g_{11}=\frac{\rho^{2}}{\Delta_{r}}, \\
& g_{33}=\frac{\left(r^{2}+a^{2}\right) \sin ^{2} \theta}{\Xi_{a}}+\frac{a\left[a\left(2 m \rho^{2}-q^{2}\right)+2 b q \rho^{2}\right] \sin ^{4} \theta}{\rho_{\theta}}, \\
& g_{44}=\frac{\left(r^{2}+b^{2}\right) \cos ^{2} \theta}{\Xi_{b}}+\frac{b\left[b\left(2 m \rho^{2}-q^{2}\right)+2 a q \rho^{2}\right] \cos ^{4} \theta}{\rho^{4} \Xi_{b}^{2}} \\
& g_{34}=g_{43}=\frac{\left[a b\left(2 m \rho^{2}-q^{2}\right)+\left(a^{2}+b^{2}\right) q \rho^{2}\right] \sin ^{2} \theta \cos ^{2} \theta}{\rho^{4} \Xi_{a} \Xi_{b}} .
\end{aligned}
$$

A central element in our approach is a near-horizon limit following the prescription of Bardeen and Horowitz [21] to zoom into a near-horizon region, and at the same time we move to a rotating frame by implementing the following coordinate change

$$
r \rightarrow r_{0}+\lambda \widetilde{r}, \quad t \rightarrow \frac{\tilde{t}}{\lambda}, \quad \phi \rightarrow \widetilde{\phi}+g \frac{\widetilde{t}}{\lambda}, \quad \psi \rightarrow \widetilde{\psi}+g \frac{\widetilde{t}}{\lambda} .
$$


Taking $\lambda \rightarrow 0$ brings us to a particular near-horizon region of the $\mathrm{AdS}_{5}$ BPS black holes given by the following metric in the coordinates $(\widetilde{t}, \widetilde{r}, \theta, \widetilde{\phi}, \widetilde{\psi})$

$$
\begin{aligned}
d s^{2}= & -\frac{2 g(1+5 a g)}{a(1+a g)^{2}} \widetilde{r}^{2} d \widetilde{t}^{2}+\frac{a}{2 g(1+5 a g)} \frac{d \widetilde{r}^{2}}{\widetilde{r}^{2}}+\Lambda_{\mathrm{AdS}_{5}}(\theta)\left[d \widetilde{\phi}+\frac{3 g(1-a g)}{(1+a g) \sqrt{a\left(a+\frac{2}{g}\right)}} \widetilde{r} d \widetilde{t}\right]^{2} \\
& +\frac{a(4-a g+3 a g \cos (2 \theta)) \cos ^{2} \theta}{2 g(1-a g)^{2}}\left[d \widetilde{\psi}+\frac{6 a g \sin ^{2} \theta}{4-a g+3 a g \cos (2 \theta)} d \widetilde{\phi}+V(\theta) \widetilde{r} d \widetilde{t}\right]^{2} \\
& +\frac{2 a}{g(1-a g)} d \theta^{2},
\end{aligned}
$$

where

$$
\begin{aligned}
\Lambda_{\mathrm{AdS}_{5}}(\theta) & \equiv \frac{4 a(2+a g) \sin ^{2} \theta}{g(1-a g)(4-a g+3 a g \cos (2 \theta))}, \\
V(\theta) & \equiv \frac{6 g^{2}(1-a g) \sqrt{a\left(a+\frac{2}{g}\right)}}{a(1+a g)(4-a g+3 a g \cos (2 \theta))},
\end{aligned}
$$

and for simplicity, we have set $a=b$, in consistency with the gravitational Cardy limit (2.18) that will be imposed later. For some special values of $\theta$, the metric (2.24) has the topology of two $\mathrm{U}(1)$ circles fibered over the $\mathrm{AdS}_{2}$ parametrized by $(\tilde{t}, \tilde{r})$, as pointed out in $[19,20]$.

After a further change of coordinates

$$
\tau \equiv \frac{2 g(1+5 a g)}{a(1+a g)} \widetilde{t},
$$

we can bring the metric (2.24) into the form

$$
\begin{aligned}
d s^{2}= & \frac{a}{2 g(1+5 a g)}\left[-\widetilde{r}^{2} d \tau^{2}+\frac{d \widetilde{r}^{2}}{\widetilde{r}^{2}}\right]+\Lambda_{\mathrm{AdS}_{5}}(\theta)\left[d \widetilde{\phi}+\frac{3 a(1-a g)}{2(1+5 a g) \sqrt{a\left(a+\frac{2}{g}\right)}} \widetilde{r} d \tau\right]^{2} \\
& +\frac{a(4-a g+3 a g \cos (2 \theta)) \cos ^{2} \theta}{2 g(1-a g)^{2}}\left[d \tilde{\psi}+\frac{6 a g \sin ^{2} \theta}{4-a g+3 a g \cos (2 \theta)} d \widetilde{\phi}+\widetilde{V}(\theta) \widetilde{r} d \tau\right]^{2} \\
& +\frac{2 a}{g(1-a g)} d \theta^{2}
\end{aligned}
$$

where

$$
\widetilde{V}(\theta) \equiv \frac{3 g(1-a g) \sqrt{a\left(a+\frac{2}{g}\right)}}{(1+5 a g)(4-a g+3 a g \cos (2 \theta))} .
$$

In both $\mathrm{U}(1)$ fibrations, the coefficients in front of $\widetilde{r} d \tau$ are proportional to $\partial_{T} \Omega$ (2.16) with $a=b$. Hence, according to the relation (2.14), $\omega_{i}$ from $\mathcal{N}=4 \mathrm{SYM}$ indeed play the role of angular velocities in the metric (2.28), and the Cardy-like limit from the field 
theory side means the angular velocities slow down on some $\mathrm{U}(1)$ circles in the near-horizon metric (2.28).

In appendix A.1, we verify explicitly that the resulting background is a solution of the $5 \mathrm{~d}$ minimal gauged supergravity equations of motion. This statement holds for arbitrary values of $a=b$. Up to this point, our approach is completely rigorous and verifying the equations of motion explicitly provides a powerful seal of approval. However, to flesh out the scaling properties of the solution, in what follows we implement the gravitational Cardy limit in the space of parameters which further simplifies the geometry.

We apply the gravitational Cardy limit (2.18) to the metric (2.28) and keep the leading orders in $\epsilon$, which leads to

$$
\begin{aligned}
d s^{2}= & \frac{1}{12 g^{2}}\left[-\widetilde{r}^{2} d \tau^{2}+\frac{d \widetilde{r}^{2}}{\widetilde{r}^{2}}\right]-\frac{2}{g^{3} \epsilon} d \theta^{2}-\frac{4 \sin ^{2}(\theta) \epsilon}{g^{3}(1+\cos (2 \theta))}\left[\frac{1}{\epsilon} d \widetilde{\phi}-\frac{g}{4 \sqrt{3}} \widetilde{r} d \tau\right]^{2} \\
& +\frac{3 \cos ^{4}(\theta)}{g^{4}}\left[\frac{1}{\epsilon} d \widetilde{\psi}+\frac{2 \sin ^{2}(\theta)}{\epsilon(1+\cos (2 \theta))} d \widetilde{\phi}-\frac{g \sec ^{2}(\theta)}{4 \sqrt{3}} \widetilde{r} d \tau\right]^{2} .
\end{aligned}
$$

From this metric, we can see that in the gravitational Cardy limit $\epsilon \rightarrow 0$ only one $\mathrm{U}(1)$ circle remains non-trivially fibered over $\mathrm{AdS}_{2}$. We have only assumed that $\epsilon$ is small without strictly taking the limit $\epsilon \rightarrow 0$, and the near-horizon metric will approximate to $\mathrm{AdS}_{3}$, as $\epsilon$ becomes smaller. However, since the two initial U(1) fibrations give the same result of the black hole entropy according to the Cardy formula and the extreme black hole/CFT correspondence [20], the remaining U(1) is enough to compute the $\mathrm{AdS}_{5}$ black hole entropy. We will demonstrate this point in the next subsection. To summarize, the gravitational Cardy limit simplifies the near-horizon geometry but keeps the minimal amount of information for computing the black hole entropy.

Let us finish by warning the potentially puzzled reader. The analysis above, surrounding equation (2.30), is local and has the sole intention of clarifying the geometry of the gravitational Cardy limit. If bothered by this last limiting procedure it is possible to step back and derive all the quantities from the safer background obtained in equation (2.28). However, without this gravitational Cardy limit the connection to the field theory approach would be very tenuous.

\subsection{Black hole entropy from Cardy formula}

In the previous subsection, we showed that a warped $\mathrm{AdS}_{3}$ geometry appears in the nearhorizon region of asymptotically $\mathrm{AdS}_{5}$ BPS black holes in the gravitational Cardy limit. This circumstance permits the use of ideas presented in [18], which lead to the identification of a Virasoro algebra as the asymptotic symmetries in the near-horizon geometry and, subsequently, to a microscopic description of the black hole entropy via the Cardy formula.

Let us briefly review how the Virasoro algebra emerges as the algebra of asymptotic symmetries of the near-horizon region of the extremal Kerr black hole [18] (see also [42]). Recall that the asymptotic symmetry group is the group of all allowed diffeomorphisms modulo trivial ones where allowed diffeomorphisms are defined as those that preserve certain boundary conditions of the asymptotic metric. The starting element in determining 
the algebra of asymptotic symmetries is, therefore, to consider diffeomorphims generated by vectors of the form

$$
\zeta_{\epsilon}=\epsilon(\phi) \frac{\partial}{\partial \phi}-r \epsilon^{\prime}(\phi) \frac{\partial}{\partial r},
$$

where $\epsilon(\phi)$ is a function periodic in $\phi$. For simplicity we can choose to be $\epsilon(\phi)=-e^{-i n \phi}$, and consequently obtain the mode expansion of $\zeta_{\epsilon}$ as

$$
\zeta_{(n)}=-e^{-i n \phi} \frac{\partial}{\partial \phi}-i n r e^{-i n \phi} \frac{\partial}{\partial r},
$$

which satisfies a centreless Virasoro algebra

$$
i\left[\zeta_{(m)}, \zeta_{(n)}\right]=(m-n) \zeta_{(m+n)} .
$$

The charge associated with the diffeomorphis $\zeta_{\epsilon}$ is given by an integral over the boundary of a spatial slice $\partial \Sigma$

$$
Q_{\zeta}=\frac{1}{8 \pi G} \int_{\partial \Sigma} k_{\zeta},
$$

where $k_{\zeta}$ is a 2 -form defined for a general perturbation $h_{\mu \nu}$ around the background metric $g_{\mu \nu}$

$$
\begin{aligned}
k_{\zeta}[h, g] \equiv-\frac{1}{4} \epsilon_{\alpha \beta \mu \nu} & {\left[\zeta^{\nu} D^{\mu} h-\zeta^{\nu} D_{\sigma} h^{\mu \sigma}+\zeta_{\sigma} D^{\nu} h^{\mu \sigma}+\frac{1}{2} h D^{\nu} \zeta^{\mu}-h^{\nu \sigma} D_{\sigma} \zeta^{\mu}\right.} \\
& \left.+\frac{1}{2} h^{\sigma \nu}\left(D^{\mu} \zeta_{\sigma}+D_{\sigma} \zeta^{\mu}\right)\right] d x^{\alpha} \wedge d x^{\beta}
\end{aligned}
$$

with $h \equiv h_{\alpha \beta} g^{\alpha \beta}$. The Dirac bracket of the charges is

$$
\left\{Q_{\zeta_{(m)}}, Q_{\zeta_{(n)}}\right\}=Q_{\left[\zeta_{(m)}, \zeta_{(n)}\right]}+\frac{1}{8 \pi G} \int_{\partial \Sigma} k_{\zeta}\left[\mathcal{L}_{\zeta} g, g\right],
$$

where $\mathcal{L}_{\zeta}$ denotes the Lie derivative with respect to $\zeta$

$$
\mathcal{L}_{\zeta} g_{\mu \nu} \equiv \zeta^{\rho} \partial_{\rho} g_{\mu \nu}+g_{\rho \nu} \partial_{\mu} \zeta^{\rho}+g_{\mu \rho} \partial_{\nu} \zeta^{\rho} .
$$

The mode expansion of the Dirac bracket (2.36) leads to a Virasora algebra

$$
\left[L_{m}, L_{n}\right]=(m-n) L_{m+n}+\frac{1}{12} c_{L}\left(m^{3}+\alpha m\right) \delta_{m+n, 0},
$$

where $c_{L}$ can be obtained from the integral

$$
\frac{1}{8 \pi G} \int_{\partial \Sigma} k_{\zeta_{(m)}}\left[\mathcal{L}_{\zeta_{(n)}} g, g\right]=-\frac{i}{12} c_{L}\left(m^{3}+\alpha m\right) \delta_{m+n, 0},
$$

and $\alpha$ is an irrelevant constant.

To compute the black hole entropy using the Cardy formula, we still need the FrolovThorne temperature, which can be obtained in the following way. The quantum fields on the background (2.1) can be expanded in the modes $e^{-i \omega t+i m \phi}$. After taking the scaling (2.23), these modes become

$$
e^{-i \omega t+i m \phi}=e^{-i \omega \frac{\widetilde{t}}{\lambda}+i m\left(\widetilde{\phi}+g \frac{\widetilde{t}}{\lambda}\right)}=e^{-i\left(\frac{\omega}{\lambda}-\frac{m g}{\lambda}\right) \widetilde{t}+i m \widetilde{\phi}} \equiv e^{-i n_{R} \widetilde{t}+i n_{L} \widetilde{\phi}},
$$


from which we can read off the left-moving and the right-moving mode numbers

$$
n_{L} \equiv m, \quad n_{R} \equiv \frac{\omega-m g}{\lambda} .
$$

The Boltzmann factor is

$$
e^{-\frac{\omega-m \Omega}{T_{H}}}=e^{-\frac{n_{L}}{T_{L}}-\frac{n_{R}}{T_{R}}},
$$

where $T_{H}$ is the Hawking temperature, and $T_{L, R}$ are the left-moving and the right-moving Frolov-Thorne temperatures. Combining (2.41) and (2.42), we obtain the near-extremal Frolov-Thorne temperatures

$$
T_{L}=\frac{T_{H}}{g-\Omega}, \quad T_{R}=\frac{T_{H}}{\lambda} .
$$

The values for the extremal $\mathrm{AdS}_{5}$ black holes can be obtained by taking the extremal limit $\left(T_{H} \rightarrow 0\right)$.

In order to apply the technique described above, we need to first transform the $\mathrm{AdS}_{2}$ Poincaré coordinates $(\widetilde{r}, \tau)$ in the metric $(2.28)$ to global coordinates $(\hat{r}, \hat{t})$

$$
g \widetilde{r}=\hat{r}+\sqrt{1+\hat{r}^{2}} \cos (\hat{t}), \quad g^{-1} \tau=\frac{\sqrt{1+\hat{r}^{2}} \sin (\hat{t})}{\hat{r}+\sqrt{1+\hat{r}^{2}} \cos (\hat{t})},
$$

which leads to

$$
\begin{aligned}
-\widetilde{r}^{2} d \tau^{2}+\frac{d \widetilde{r}^{2}}{\widetilde{r}^{2}} & =-\left(1+\hat{r}^{2}\right) d \hat{t}^{2}+\frac{d \hat{r}^{2}}{1+\hat{r}^{2}} \\
\widetilde{r} d \tau & =\hat{r} d \hat{t}+d \gamma
\end{aligned}
$$

where

$$
\gamma \equiv \log \left(\frac{1+\sqrt{1+\hat{r}^{2}} \sin (\hat{t})}{\cos (\hat{t})+\hat{r} \sin (\hat{t})}\right) .
$$

Consequently, the near-horizon metric (2.28) of the $\mathrm{AdS}_{5}$ BPS black holes can be written as

$$
\begin{aligned}
d s^{2}= & \frac{a}{2 g(1+5 a g)}\left[-\left(1+\hat{r}^{2}\right) d \hat{t}^{2}+\frac{d \hat{r}^{2}}{1+\hat{r}^{2}}\right]+\Lambda_{\mathrm{AdS}_{5}}(\theta)\left[d \hat{\phi}+\frac{3 a(1-a g)}{2(1+5 a g) \sqrt{a\left(a+\frac{2}{g}\right)}} \hat{r} d \hat{t}\right]^{2} \\
& +\frac{a(4-a g+3 a g \cos (2 \theta)) \cos ^{2} \theta}{2 g(1-a g)^{2}}\left[d \hat{\psi}+\frac{6 a g \sin ^{2} \theta}{4-a g+3 a g \cos (2 \theta)} d \hat{\phi}+\tilde{V}(\theta) \hat{r} d \hat{t}\right]^{2} \\
& +\frac{2 a}{g(1-a g)} d \theta^{2}
\end{aligned}
$$

where

$$
\hat{\phi} \equiv \widetilde{\phi}+\frac{3 a(1-a g) \gamma}{2(1+5 a g) \sqrt{a\left(a+\frac{2}{g}\right)}}, \quad \hat{\psi} \equiv \widetilde{\psi}+\frac{3 a(1-a g) \gamma}{2(1+5 a g) \sqrt{a\left(a+\frac{2}{g}\right)}} .
$$


Applying the formalism reviewed in this subsection, we can compute the central charge and the extremal Frolov-Thorne temperature in the near-horizon region of the asymptotically $\mathrm{AdS}_{5}$ BPS black hole solutions (2.47)

$$
\begin{aligned}
c_{L} & =\frac{9 \pi a^{2}}{g(1-a g)(1+5 a g)}, \\
T_{L} & =\frac{1+5 a g}{3 a(1-a g) \pi} \sqrt{a\left(a+\frac{2}{g}\right)} .
\end{aligned}
$$

The BPS black hole entropy in this case is then given by the Cardy formula

$$
S_{B H}=\frac{\pi^{2}}{3} c_{L} T_{L}=\frac{\pi^{2} a \sqrt{2 a+a^{2} g}}{g^{3 / 2}(1-a g)^{2}},
$$

which is the same as the result from gravity (2.8) with $a=b$. In fact, we can also apply the formalism discussed in this subsection to the near-horizon metric in the gravitational Cardy limit (2.30), which can be recast into the global coordinates

$$
\begin{aligned}
d s^{2}= & \frac{1}{12 g^{2}}\left[-\left(1+\hat{r}^{2}\right) d \hat{t}^{2}+\frac{d \hat{r}^{2}}{1+\hat{r}^{2}}\right]-\frac{2}{g^{3} \epsilon} d \theta^{2}-\frac{4 \sin ^{2}(\theta) \epsilon}{g^{3}(1+\cos (2 \theta))}\left[\frac{1}{\epsilon} d \hat{\phi}-\frac{g}{4 \sqrt{3}} \hat{r} d \hat{t}\right]^{2} \\
& +\frac{3 \cos ^{4}(\theta)}{g^{4}}\left[\frac{1}{\epsilon} d \hat{\psi}+\frac{2 \sin ^{2}(\theta)}{\epsilon(1+\cos (2 \theta))} d \hat{\phi}-\frac{g \sec ^{2}(\theta)}{4 \sqrt{3}} \hat{r} d \hat{t}\right]^{2} .
\end{aligned}
$$

The corresponding central charge and the extremal Frolov-Thorne temperature are

$$
c_{L}=\frac{3 \pi}{2 g^{4} \epsilon}, \quad T_{L}=\frac{2 \sqrt{3}}{\pi g \epsilon} .
$$

The black hole entropy can obtained from the Cardy formula

$$
S_{B H}=\frac{\pi^{2}}{3} c_{L} T_{L}=\frac{\sqrt{3} \pi^{2}}{g^{5} \epsilon^{2}}
$$

which exactly matches the gravity result in the gravitational Cardy limit (2.19).

\subsection{Comparison with results from boundary CFT}

The asymptotically $\mathrm{AdS}_{5}$ BPS black hole entropy can also be obtained from the boundary $\mathcal{N}=4$ SYM by extremizing an entropy function [3-5] originally motivated in [43] and more recently studied in [44]. One can first compute the free energy in the large- $N$ limit using the partition function via localization or the $4 \mathrm{~d}$ superconformal index. The entropy function is then defined as the Legendre transform of the free energy in the large- $N$ limit

$$
S\left(\Delta_{I}, \omega\right)=\frac{N^{2}}{2} \frac{\Delta_{1} \Delta_{2} \Delta_{3}}{\omega_{1} \omega_{2}}+\sum_{I=1}^{3} Q_{I} \Delta_{I}+\sum_{i=1}^{2} J_{i} \omega_{i}-\Lambda\left(\sum_{I} \Delta_{I}-\sum_{i} \omega-2 \pi i\right) .
$$

In the Cardy-like limit (2.13)

$$
\omega \sim \epsilon, \quad \Delta_{I} \sim \mathcal{O}(1)
$$


we can read off from the entropy function (2.55)

$$
S \sim \frac{1}{\epsilon^{2}}, \quad J \sim \frac{1}{\epsilon^{3}}, \quad Q_{I} \sim \frac{1}{\epsilon^{2}},
$$

which have been summarized in table 1 .

The electric charges $Q_{I}$ and the angular momenta $J_{i}$ are real, while the chemical potentials $\Delta_{I}$ and the angular velocities $\omega_{i}$ can be complex, and so can the entropy function $S$. By requiring that the black hole entropy $S_{B H}$ be real after extremizing the entropy function $S$, we obtain one more constraint on $Q_{I}$ and $J_{i}$. More precisely, the asymptotically $\mathrm{AdS}_{5}$ black hole entropy is given by $[3-5]$

$$
S_{B H}=2 \pi \sqrt{Q_{1} Q_{2}+Q_{2} Q_{3}+Q_{3} Q_{1}-\frac{N^{2}}{2}\left(J_{1}+J_{2}\right)},
$$

subject to the constraint

$$
\begin{aligned}
\left(Q_{1}+Q_{2}+Q_{3}+\frac{N^{2}}{2}\right)\left(Q_{1} Q_{2}+Q_{2} Q_{3}\right. & \left.+Q_{3} Q_{1}-\frac{N^{2}}{2}\left(J_{1}+J_{2}\right)\right) \\
& -\left(Q_{1} Q_{2} Q_{3}+\frac{N^{2}}{2} J_{1} J_{2}\right)=0
\end{aligned}
$$

which is a consequence of the reality condition on the black hole entropy $S_{B H}$.

For the $\mathrm{AdS}_{5}$ black hole solutions in [29], the electric charges are degenerate, i.e. $Q_{1}=Q_{2}=Q_{3}=Q$. Hence, for this class of black hole solutions in the BPS limit, the black hole entropy becomes

$$
S_{B H}=2 \pi \sqrt{3 Q^{2}-\frac{N^{2}}{2}\left(J_{1}+J_{2}\right)} .
$$

This is exactly the same as the result from the horizon area (2.12) in the unit $g=1$, and the one from the Cardy formula (2.51). The constraint (2.59) for this degenerate case becomes

$$
\left(3 Q+\frac{N^{2}}{2}\right)\left(3 Q^{2}-\frac{N^{2}}{2}\left(J_{1}+J_{2}\right)\right)=Q^{3}+\frac{N^{2}}{2} J_{1} J_{2},
$$

which is also consistent with the thermodynamic quantities from the gravity side (2.8).

\section{Asymptotically $\mathrm{AdS}_{4}$ black holes}

In this section, we consider the asymptotically $\mathrm{AdS}_{4}$ black holes and the corresponding gravitational Cardy limit. Similar to the $\mathrm{AdS}_{5}$ case, we demonstrate that the $\mathrm{AdS}_{4}$ black hole entropy can be computed in various ways as shown in figure 1 , and the other thermodynamic quantities scale correspondingly (see table 1) in the gravitational Cardy limit. 


\section{1 $\quad \mathrm{AdS}_{4}$ black hole solution}

The non-extremal rotating, electrically charged asymptotically $\mathrm{AdS}_{4}$ black hole solution with gauge group $\mathrm{U}(1) \times \mathrm{U}(1)$ in $4 \mathrm{~d} \mathcal{N}=4$ gauged supergravity was constructed in [45]. The solution is characterized by four parameters $\left(a, m, \delta_{1}, \delta_{2}\right)$. The metric, the scalars and the gauge fields are given by

$$
\begin{aligned}
d s^{2} & =-\frac{\Delta_{r}}{W}\left(d t-\frac{a \sin ^{2} \theta}{\Xi} d \phi\right)^{2}+W\left(\frac{d r^{2}}{\Delta_{r}}+\frac{d \theta^{2}}{\Delta_{\theta}}\right)+\frac{\Delta_{\theta} \sin ^{2} \theta}{W}\left[a d t-\frac{r_{1} r_{2}+a^{2}}{\Xi} d \phi\right]^{2}, \\
e^{\varphi_{1}} & =\frac{r_{1}^{2}+a^{2} \cos ^{2} \theta}{W}, \quad \chi_{1}=\frac{a\left(r_{2}-r_{1}\right) \cos \theta}{r_{1}^{2}+a^{2} \cos ^{2} \theta} \\
A_{1} & =\frac{2 \sqrt{2} m \sinh \left(\delta_{1}\right) \cosh \left(\delta_{1}\right) r_{2}}{W}\left(d t-\frac{a \sin ^{2} \theta}{\Xi} d \phi\right)+\alpha_{41} d t \\
A_{2} & =\frac{2 \sqrt{2} m \sinh \left(\delta_{2}\right) \cosh \left(\delta_{2}\right) r_{1}}{W}\left(d t-\frac{a \sin ^{2} \theta}{\Xi} d \phi\right)+\alpha_{42} d t
\end{aligned}
$$

where

$$
\begin{aligned}
r_{i} & \equiv r+2 m \sinh ^{2}\left(\delta_{i}\right), \quad(i=1,2) \\
\Delta_{r} & \equiv r^{2}+a^{2}-2 m r+g^{2} r_{1} r_{2}\left(r_{1} r_{2}+a^{2}\right), \\
\Delta_{\theta} & \equiv 1-g^{2} a^{2} \cos ^{2} \theta, \\
W & \equiv r_{1} r_{2}+a^{2} \cos ^{2} \theta, \\
\Xi & \equiv 1-a^{2} g^{2},
\end{aligned}
$$

and $g \equiv \ell_{4}^{-1}$ is the inverse of the $\mathrm{AdS}_{4}$ radius. Note that we have added pure gauge terms to the two gauge fields where $\alpha_{41}$ and $\alpha_{42}$ are constant. The metric (3.1) can also be written in the following equivalent expression, which is more convenient for later discussions,

$$
d s^{2}=-\frac{\Delta_{r} \Delta_{\theta}}{B \Xi^{2}} d t^{2}+B \sin ^{2} \theta(d \phi+f d t)^{2}+W\left(\frac{d r^{2}}{\Delta_{r}}+\frac{d \theta^{2}}{\Delta_{\theta}}\right),
$$

with

$$
\begin{gathered}
B \equiv \frac{\left(a^{2}+r_{1} r_{2}\right)^{2} \Delta_{\theta}-a^{2} \sin ^{2}(\theta) \Delta_{r}}{W \Xi^{2}}, \\
f \equiv \frac{a \Xi\left(\Delta_{r}-\Delta_{\theta}\left(a^{2}+r_{1} r_{2}\right)\right)}{\Delta_{\theta}\left(a^{2}+r_{1} r_{2}\right)^{2}-a^{2} \Delta_{r} \sin ^{2} \theta} .
\end{gathered}
$$

The non-extremal asymptotically $\mathrm{AdS}_{4}$ black holes with four degenerate electric charges $\left(Q_{1}=Q_{2}, Q_{3}=Q_{4}\right)$ and one angular momentum $J$ have been found in [46], which are characterized by four parameters $\left(a, m, \delta_{1}, \delta_{2}\right)$. The BPS limit imposes a condition

$$
e^{2 \delta_{1}+2 \delta_{2}}=1+\frac{2}{a g} \text {. }
$$


For the black hole solution to have a regular horizon, we impose an additional constraint

$$
(m g)^{2}=\frac{\cosh ^{2}\left(\delta_{1}+\delta_{2}\right)}{e^{\delta_{1}+\delta_{2}} \sinh ^{3}\left(\delta_{1}+\delta_{2}\right) \sinh \left(2 \delta_{1}\right) \sinh \left(2 \delta_{2}\right)} .
$$

The two conditions (3.6) (3.7) in [46] have typos, which have been corrected in [47, 48], see also [49]. With these constraints, there are only two independent parameters for asymptotically $\mathrm{AdS}_{4}$ BPS black holes, which we choose to be $\left(\delta_{1}, \delta_{2}\right)$ for convenience. In the BPS limit, the position of the outer horizon is

$$
r_{+}=\frac{2 m \sinh \left(\delta_{1}\right) \sinh \left(\delta_{2}\right)}{\cosh \left(\delta_{1}+\delta_{2}\right)}
$$

which coincides with the inner horizon.

The physical quantities of non-extremal $\mathrm{AdS}_{4}$ black holes can also be solved as functions of $\left(a, m, \delta_{1}, \delta_{2}\right)$. In particular, the gravitational angular velocity $\Omega$ and the temperature $T$ are given by

$$
\Omega=\frac{a\left(1+g^{2} r_{1} r_{2}\right)}{r_{1} r_{2}+a^{2}}, \quad T=\frac{\Delta_{r}^{\prime}}{4 \pi\left(r_{1} r_{2}+a^{2}\right)} .
$$

Moreover, the other thermodynamic quantities of asymptotically $\mathrm{AdS}_{4}$ black holes are [46]

$$
\begin{aligned}
S & =\frac{\pi\left(r_{1} r_{2}+a^{2}\right)}{\Xi}, \\
J & =\frac{m a}{2 \Xi^{2}}\left(\cosh \left(2 \delta_{1}\right)+\cosh \left(2 \delta_{2}\right)\right), \\
Q_{1}=Q_{2} & =\frac{m}{4 \Xi} \sinh \left(2 \delta_{1}\right), \\
Q_{3}=Q_{4} & =\frac{m}{4 \Xi} \sinh \left(2 \delta_{2}\right) .
\end{aligned}
$$

\subsection{Gravitational Cardy limit}

The Cardy-like limit for the $3 \mathrm{~d}$ ABJM theory was defined in $[9,10]$,

$$
|\omega| \ll 1, \quad \Delta_{I} \sim \mathcal{O}(1), \quad(I=1, \cdots, 4) .
$$

Using the relations found in [48]

$$
\omega=-\lim _{T \rightarrow 0} \frac{\Omega-\Omega^{*}}{T}, \quad \Delta_{I}=-\lim _{T \rightarrow 0} \frac{\Phi_{I}-\Phi_{I}^{*}}{T},
$$

with $\Omega^{*}=g$ and $\Phi_{I}^{*}=1$ denoting the BPS values of $\Omega$ and $\Phi_{I}$, we can find the gravitational counterpart of the Cardy-like limit (3.11)

$$
\left|\left(\frac{\partial \Omega}{\partial T}\right)_{T=0}\right| \ll 1,\left.\quad \frac{\partial \Phi_{I}}{\partial T}\right|_{T=0} \sim \mathcal{O}(1) .
$$

Hence, we obtain for the near-extremal $\mathrm{AdS}_{4}$ black holes

$$
\left.\frac{\partial \Omega}{\partial T}\right|_{\mathrm{BPS}}=\lim _{T \rightarrow 0} \frac{\Omega-\Omega_{*}}{T}=-\frac{\pi e^{\frac{5}{2}\left(\delta_{1}+\delta_{2}\right)}\left(\operatorname{coth}\left(\delta_{1}+\delta_{2}\right)-2\right) \sqrt{\sinh \left(2 \delta_{1}\right) \sinh \left(2 \delta_{2}\right)}}{\left(\operatorname{coth}\left(\delta_{1}+\delta_{2}\right)+1\right) \sqrt{\sinh \left(\delta_{1}+\delta_{2}\right)} \cosh \left(\delta_{1}-\delta_{2}\right)} .
$$


This expression has several roots

$$
\delta_{1}=0, \quad \delta_{2}=0, \quad \delta_{1}+\delta_{2}=\operatorname{arccoth}(2) .
$$

However, $\delta_{1}=0$ and $\delta_{2}=0$ are unphysical, because according to (3.8), $\delta_{1}=0$ or $\delta_{2}=0$ will cause $r_{+} \rightarrow 0$. Hence, we conclude that the gravitational Cardy limit for asymptotically $\mathrm{AdS}_{4}$ BPS black holes is

$$
\delta_{1}+\delta_{2} \rightarrow \operatorname{arccoth}(2) .
$$

Equivalently, this can be written in terms of the other parameters as

$$
a g \rightarrow 1 .
$$

We introduce a small parameter $\epsilon$ to denote small deviations from this limit, i.e.,

$$
\delta_{1}+\delta_{2}=\operatorname{arccoth}(2)+\epsilon .
$$

For this case $\epsilon$ is dimensionless. Imposing first the BPS constraint (3.6) and the regularity condition (3.7) near the horizon, and then taking the gravitational Cardy limit (3.18), we obtain the thermodynamic quantities (3.10) to the leading order in $\epsilon$

$$
\begin{aligned}
S_{*} & =\frac{\pi}{3 g^{2} \epsilon}+\mathcal{O}(1), \\
J_{*} & =\frac{\cosh \left(2 \delta_{1}-\frac{1}{2} \log (3)\right)}{9 g^{2} \epsilon^{2} \sqrt{2 \sinh \left(4 \delta_{1}\right)-5 \sinh ^{2}\left(2 \delta_{1}\right)}}+\mathcal{O}\left(\epsilon^{-1}\right), \\
Q_{1}^{*}=Q_{2}^{*} & =\frac{1}{4 g \epsilon \sqrt{6 \tanh \left(\delta_{1}\right)+6 \operatorname{coth}\left(\delta_{1}\right)-15}}+\mathcal{O}(1), \\
Q_{3}^{*}=Q_{4}^{*} & =\frac{\sqrt{2 \tanh \left(\delta_{1}\right)+2 \operatorname{coth}\left(\delta_{1}\right)-5}}{12 \sqrt{3} g \epsilon}+\mathcal{O}(1),
\end{aligned}
$$

which are consistent with $[9,41]$ and the Cardy-like limit on the field theory side (3.11)

$$
\omega_{*} \sim \epsilon, \quad \Delta_{I *} \sim \mathcal{O}(1)
$$

\subsection{Black hole solution in the near-horizon + gravitational Cardy limit}

In the previous subsection, we have obtained the gravitational Cardy limit for the parameters on the gravity side. In this subsection, we discuss how the near-horizon metric changes when taking the gravitational Cardy limit. In appendix A.2, we verify explicitly that the resulting background is a solution of the $4 \mathrm{~d}$ gauged supergravity equations of motion. In the following, we implement the gravitational Cardy limit in the space of parameters, which further simplifies the geometry.

For the asymptotically $\mathrm{AdS}_{4}$ black hole metric (3.4), we perform a near-horizon scaling similar to the $\mathrm{AdS}_{5}$ case (2.23)

$$
r \rightarrow r_{*}+\lambda \widetilde{r}, \quad t \rightarrow \frac{\tilde{t}}{\lambda}, \quad \phi \rightarrow \widetilde{\phi}-g\left[\operatorname{coth}\left(\delta_{1}+\delta_{2}\right)-2\right] \frac{\tilde{t}}{\lambda} .
$$


Furthermore, we take the gravitational Cardy limit (3.18) and keep only the leading orders in $\epsilon$. The metric (3.4) finally becomes

$$
\begin{aligned}
d s^{2}= & -\frac{g^{2}\left(9-18 e^{4 \delta_{1}}+e^{8 \delta_{1}}\right)(3+\cos (2 \theta))}{3\left(9-10 e^{4 \delta_{1}}+e^{8 \delta_{1}}\right)} \widetilde{r}^{2} d \widetilde{t}^{2}+\frac{3(3+\cos (2 \theta))\left(e^{4 \delta_{1}}-1\right)\left(e^{4 \delta_{1}}-9\right)}{16 g^{2}\left(9-18 e^{4 \delta_{1}}+e^{8 \delta_{1}}\right)} \frac{d \widetilde{r}^{2}}{\widetilde{r}^{2}} \\
& +\frac{3+\cos (2 \theta)}{2 g^{2} \sin ^{2} \theta} d \theta^{2}+\frac{2 \sin ^{4} \theta}{9 g^{2}(3+\cos (2 \theta))}\left[\frac{d \widetilde{\phi}}{\epsilon}+\frac{\sqrt{3} g^{2} \cosh \left(2 \delta_{1}-\frac{1}{2} \log (3)\right)}{\sqrt{\sinh \left(2 \delta_{1}\right) \sinh \left(\log (3)-2 \delta_{1}\right)}} \widetilde{r} d \widetilde{t}\right]^{2} \\
& +\mathcal{O}(\epsilon) .
\end{aligned}
$$

Defining

$$
\tau \equiv \frac{4 g^{2}\left(9-5 \cosh \left(4 \delta_{1}\right)+4 \sinh \left(4 \delta_{1}\right)\right)^{2}}{3\left(5-5 \cosh \left(4 \delta_{1}\right)+4 \sinh \left(4 \delta_{1}\right)\right)} \widetilde{t}
$$

we can rewrite the metric $(3.22)$ as

$$
\begin{aligned}
d s^{2}= & \frac{3(3+\cos (2 \theta))\left(e^{4 \delta_{1}}-1\right)\left(e^{4 \delta_{1}}-9\right)}{16 g^{2}\left(9-18 e^{4 \delta_{1}}+e^{8 \delta_{1}}\right)}\left[-\widetilde{r}^{2} d \tau^{2}+\frac{d \widetilde{r}^{2}}{\widetilde{r}^{2}}\right]+\frac{3+\cos (2 \theta)}{2 g^{2} \sin ^{2} \theta} d \theta^{2} \\
& +\frac{2 \sin ^{4} \theta}{9 g^{2}(3+\cos (2 \theta))}\left[\frac{d \widetilde{\phi}}{\epsilon}+V\left(\delta_{1}\right) \widetilde{r} d \tau\right]^{2}+\mathcal{O}(\epsilon)
\end{aligned}
$$

where

$$
V\left(\delta_{1}\right) \equiv \frac{9 \cosh \left(2 \delta_{1}-\frac{1}{2} \log (3)\right)\left(5-5 \cosh \left(4 \delta_{1}\right)+4 \sinh \left(4 \delta_{1}\right)\right)}{2 \sqrt{10-6 \cosh \left(4 \delta_{1}-\log (3)\right)}\left(9-5 \cosh \left(4 \delta_{1}\right)+4 \sinh \left(4 \delta_{1}\right)\right)} .
$$

\subsection{Black hole entropy from Cardy formula}

For the asymptotically $\mathrm{AdS}_{4}$ black holes discussed in this section, we apply the Cardy formula to the near-horizon metric only after taking the gravitational Cardy limit. More explicitly, we first rewrite the metric (3.24) from the Poincaré coordinates $(\widetilde{r}, \tau)$ to the global coordinates $(\hat{r}, \hat{t})$ using the relations $(2.44)-(2.46)$. Consequently, the near-horizon metric in the gravitational Cardy limit (3.24) becomes

$$
\begin{aligned}
d s^{2}= & \frac{3(3+\cos (2 \theta))\left(e^{4 \delta_{1}}-1\right)\left(e^{4 \delta_{1}}-9\right)}{16 g^{2}\left(9-18 e^{4 \delta_{1}}+e^{8 \delta_{1}}\right)}\left[-\left(1+\hat{r}^{2}\right) d \hat{t}^{2}+\frac{d \hat{r}^{2}}{1+\hat{r}^{2}}\right]+\frac{3+\cos (2 \theta)}{2 g^{2} \sin ^{2} \theta} d \theta^{2} \\
& +\frac{2 \sin ^{4} \theta}{9 g^{2}(3+\cos (2 \theta))}\left[\frac{d \hat{\psi}}{\epsilon}+V\left(\delta_{1}\right) \hat{r} d \hat{t}\right]^{2}+\mathcal{O}(\epsilon),
\end{aligned}
$$

where $\hat{t}, \hat{r}$ and $\gamma$ are defined in (2.44) and (2.46), while

$$
\hat{\psi} \equiv \hat{\phi}+V\left(\delta_{1}\right) \gamma \epsilon
$$

Applying the same formalism in subsection 2.4, we obtain the central charge and the extremal Frolov-Thorne temperature in the near-horizon region of the asymptotically $\mathrm{AdS}_{4}$ 
BPS black holes,

$$
\begin{aligned}
c_{L} & =\frac{3 \sqrt{\frac{3}{2}} e^{2 \delta_{1}}\left(3+e^{4 \delta_{1}}\right) \sqrt{5+4 \sinh \left(4 \delta_{1}\right)-5 \cosh \left(4 \delta_{1}\right)}}{g^{2}\left(18 e^{4 \delta_{1}}-e^{8 \delta_{1}}-9\right)}, \\
T_{L} & =\frac{9+4 \sinh \left(4 \delta_{1}\right)-5 \cosh \left(4 \delta_{1}\right)}{18 \pi \epsilon \sinh \left(\delta_{1}\right) \cosh \left(\delta_{1}\right) \cosh \left(2 \delta_{1}-\frac{1}{2} \log (3)\right) \sqrt{2 \tanh \left(\delta_{1}\right)+2 \operatorname{coth}\left(\delta_{1}\right)-5}} .
\end{aligned}
$$

Using the Cardy formula, we can compute the black hole entropy of the asymptotically $\mathrm{AdS}_{4}$ BPS black holes:

$$
S_{B H}=\frac{\pi^{2}}{3} c_{L} T_{L}=\frac{\pi}{3 g^{2} \epsilon},
$$

which is the same as the black hole entropy in the gravitational Cardy limit (3.19) from the gravity side.

\subsection{Comparison with results from boundary CFT}

The asymptotically $\mathrm{AdS}_{4}$ BPS black hole entropy can also be obtained from the boundary 3d ABJM theory by extremizing an entropy function [9, 10], which has also been studied in [44]. One can first compute the free energy in the large- $N$ limit using the $3 \mathrm{~d}$ superconformal index or the partition function via localization. The entropy function is then defined as a Legendre transform of the free energy in the large- $N$ limit

$$
S\left(\Delta_{I}, \omega\right)=\frac{2 \sqrt{2} i k^{\frac{1}{2}} N^{\frac{3}{2}}}{3} \frac{\sqrt{\Delta_{1} \Delta_{2} \Delta_{3} \Delta_{4}}}{\omega}-2 \omega J-\sum_{I} \Delta_{I} Q_{I}-\Lambda\left(\sum_{I} \Delta_{I}-2 \omega+2 \pi i\right) .
$$

In the Cardy-like limit (3.11)

$$
\omega \sim \epsilon, \quad \Delta_{I} \sim \mathcal{O}(1)
$$

we can read off from the entropy function $(3.30)$

$$
S \sim \frac{1}{\epsilon}, \quad J \sim \frac{1}{\epsilon^{2}}, \quad Q_{I} \sim \frac{1}{\epsilon},
$$

which have been summarized in table 1 .

Similar to the $\mathrm{AdS}_{5}$ case, for $\mathrm{AdS}_{4}$ the electric charges $Q_{I}$ and the angular momentum $J$ are real, while the chemical potentials $\Delta_{I}$ and the angular velocity $\omega$ can be complex, and so can the entropy function $S$. By requiring that the black hole entropy $S_{B H}$ to be real after extremizing the entropy function $S$, we obtain one more constraint on $Q_{I}$ and $J$. More precisely, for the degenerate case with $Q_{1}=Q_{3}, Q_{2}=Q_{4}$ and one angular momentum $J$, the asymptotically $\mathrm{AdS}_{4}$ black hole entropy is given by $[9,10,48]$

$$
S_{B H}=\frac{2 \pi}{3} \sqrt{\frac{9 Q_{1} Q_{2}\left(Q_{1}+Q_{2}\right)-2 k J N^{3}}{Q_{1}+Q_{2}}},
$$

subject to the constraint

$$
2 k J^{2} N^{3}+2 k J N^{3}\left(Q_{1}+Q_{2}\right)-9 Q_{1} Q_{2}\left(Q_{1}+Q_{2}\right)^{2}=0,
$$

which is a consequence of the reality condition on the black hole entropy $S_{B H}$. 
If we identify the field theory parameters with the ones on the gravity side in the following way $[48,50]$

$$
\frac{1}{G}=\frac{2 \sqrt{2}}{3} g^{2} k^{\frac{1}{2}} N^{\frac{3}{2}}, \quad Q_{B H}=\frac{g}{2} Q, \quad J_{B H}=J,
$$

we can rewrite the black hole entropy (3.33) and the angular momentum as

$$
\begin{aligned}
S_{B H} & =\frac{\pi}{g^{2} G} \frac{J_{B H}}{\left(\frac{2}{g} Q_{B H, 1}+\frac{2}{g} Q_{B H, 2}\right)}, \\
J_{B H} & =\frac{1}{2}\left(\frac{2}{g} Q_{B H, 1}+\frac{2}{g} Q_{B H, 2}\right)\left(-1+\sqrt{1+16 g^{4} G^{2} \frac{2 Q_{B H, 1}}{g} \frac{2 Q_{B H, 2}}{g}}\right),
\end{aligned}
$$

which are consistent with both the thermodynamic quantities on the gravity side (3.10) (3.19) and the black hole entropy in the gravitational Cardy limit from the Cardy formula (3.29).

\section{Asymptotically $\mathrm{AdS}_{7}$ black holes}

In this section, we consider asymptotically $\mathrm{AdS}_{7}$ black holes and the corresponding gravitational Cardy limit. Similar to the previous sections, we demonstrate that the $\mathrm{AdS}_{7}$ black hole entropy can be computed in various ways as shown in figure 1, and the other thermodynamic quantities scale correspondingly in gravitational Cardy limit. For completeness, we discuss two asymptotically $\mathrm{AdS}_{7}$ black hole solutions in the literature: a special case with all equal charges and all equal angular momenta in subsection 4.1 and a more general case with two equal charges and three independent angular momenta in subsection 4.2.

\subsection{A special case}

In this subsection, we consider the gravitational Cardy limit of a special class of nonextremal asymptotically $\mathrm{AdS}_{7}$ black holes discussed in [7].

\subsection{1 $\quad \mathrm{AdS}_{7}$ black hole solution}

The solutions $\mathcal{M}_{7} \times S^{4}$ to 11 d gauged supergravity, with $\mathcal{M}_{7}$ denoting an asymptotically $\mathrm{AdS}_{7}$ black hole, have the isometry group $\mathrm{SO}(2,6) \times \mathrm{SO}(5)$. Hence, this class of solutions has three angular momenta from the Cartan of the maximal compact subgroup $\mathrm{SO}(6) \subset$ $\mathrm{SO}(2,6)$ and two electric charges from the Cartan of $\mathrm{SO}(5)$. The most generic solution has not been constructed in the literature so far. Instead, the solutions with two charges and three equal angular momenta were found in [51], while the ones with two equal charges and three angular momenta were found in [52]. As the intersection of these two classes, the solution with two equal charges $Q_{1}=Q_{2}$ and three equal angular momenta $J_{1}=J_{2}=J_{3}$ has been considered in [7].

For this special solution, the metric of the asymptotically $\mathrm{AdS}_{7}$ black hole part is given by

$$
d s_{7}^{2}=H^{\frac{2}{5}}\left[-\frac{Y}{f_{1} \Xi_{-}^{2}} d t^{2}+\frac{\Xi \rho^{6}}{Y} d r^{2}+\frac{f_{1}}{H^{2} \Xi^{2} \rho^{4}}\left(\sigma-\frac{2 f_{2}}{f_{1}} d t\right)^{2}+\frac{\rho^{2}}{\Xi} d s_{\mathbb{C P}^{2}}^{2}\right],
$$


where

$$
\begin{aligned}
\sigma & \equiv d \chi+\frac{1}{2} l_{3} \sin ^{2} \xi, \\
d s_{\mathbb{C P}^{2}}^{2} & =d \xi^{2}+\frac{1}{4} \sin ^{2} \xi\left(l_{1}^{2}+l_{2}^{2}\right)+\frac{1}{4} l_{3}^{2} \sin ^{2} \xi \cos ^{2} \xi,
\end{aligned}
$$

with $\left(l_{1}, l_{2}, l_{3}\right)$ denoting the left-invariant 1 -forms of $\mathrm{SU}(2)$, which can be explicitly chosen to be $[53]$

$$
\begin{gathered}
l_{1}=\sin \psi d \theta-\cos \psi \sin \theta d \phi, \\
l_{2}=\cos \psi d \theta+\sin \psi \sin \theta d \phi, \\
l_{3}=-(d \psi+\cos \theta d \phi), \\
0 \leq \theta \leq \pi, \quad 0 \leq \phi \leq 2 \pi, \quad 0 \leq \psi \leq 4 \pi .
\end{gathered}
$$

Moreover, the asymptotically $\mathrm{AdS}_{7}$ black hole solution also contains two 1-forms, one 3form and two scalars, which are given by

$$
\begin{aligned}
& A_{(1)}^{1}=A_{(1)}^{2}=A_{(1)}=\frac{m \sinh (\delta) \cosh (\delta)}{\rho^{4} \Xi H}(d t-a \sigma)+\frac{\alpha_{70}}{\Xi_{-}} d t \\
& A_{(3)}=\frac{m a \sinh ^{2}(\delta)}{\rho^{2} \Xi \Xi_{-}} \sigma \wedge d \sigma+\alpha_{71} d t \wedge d \theta \wedge d \psi+\alpha_{72} d t \wedge d \xi \wedge d \phi+\alpha_{73} d t \wedge d \xi \wedge d \psi, \\
& X_{1}=X_{2}=H^{-1 / 5}
\end{aligned}
$$

where we have added some pure gauge terms to the potentials, and

$$
\begin{aligned}
\rho^{2} & \equiv \Xi r^{2} \\
H & \equiv 1+\frac{2 m \sinh ^{2}(\delta)}{\rho^{4}}, \\
f_{1} & \equiv \Xi \rho^{6} H^{2}-\frac{\left[2 \Xi_{+} m a \sinh ^{2}(\delta)\right]^{2}}{\rho^{4}}+2 m a^{2}\left[\Xi_{+}^{2}+\cosh ^{2}(\delta)\left(1-\Xi_{+}^{2}\right)\right], \\
f_{2} & \equiv-\frac{g \Xi_{+} \rho^{6} H^{2}}{2}+m a \cosh ^{2}(\delta), \\
Y & \equiv g^{2} \rho^{8} H^{2}+\Xi \rho^{6}-2 m \rho^{2}\left[a^{2} g^{2} \cosh ^{2}(\delta)+\Xi\right]+2 m a^{2}\left[\Xi_{+}^{2}+\cosh ^{2}(\delta)\left(1-\Xi_{+}^{2}\right)\right], \\
\Xi_{ \pm} & \equiv 1 \pm a g, \quad \Xi \equiv 1-a^{2} g^{2},
\end{aligned}
$$

with $g=\ell_{7}^{-1}$ denoting the inverse of the $\mathrm{AdS}_{7}$ radius. The thermodynamic quantities of 
the black hole have the following expressions

$$
\begin{aligned}
T & =\frac{\partial_{r} Y}{4 \pi g \rho^{3} \sqrt{\Xi f_{1}}}, \\
S & =\frac{\pi^{3} \rho^{2} \sqrt{f_{1}}}{4 G_{N} \Xi^{3}}, \\
\Omega & =-\frac{1}{g}\left(g+\frac{2 f_{2} \Xi_{-}}{f_{1}}\right), \\
\Phi & =\frac{4 m \sinh (\delta) \cosh (\delta)}{\rho^{4} \Xi H}\left(\Xi_{-}-\frac{2 a f_{2} \Xi_{-}}{f_{1}}\right), \\
E & =\frac{m \pi^{2}}{32 G_{N} g \Xi^{4}}\left[12(a g+1)^{2}(a g(a g+2)-1)-4 \cosh ^{2}(\delta)\left(3 a^{4} g^{4}+12 a^{3} g^{3}+11 a^{2} g^{2}-8\right)\right], \\
J & =-\frac{m a \pi^{2}}{16 G_{N} \Xi^{4}}\left[4 a g(a g+1)^{2}-4 \cosh ^{2}(\delta)\left(a^{3} g^{3}+2 a^{2} g^{2}+a g-1\right)\right], \\
Q & =\frac{m \pi^{2} \sinh (\delta) \cosh (\delta)}{4 G_{N} g \Xi^{3}} .
\end{aligned}
$$

The expression of the temperature $T$ has three roots $r_{ \pm}$and $r_{0}$, all of which coincide at the extremality. As we can see, the solution depends only on three parameters $(a, m, \delta)$. As shown in [7], the BPS condition and the absence of naked closed timelike curves (CTCs) require that

$$
e^{2 \delta}=1-\frac{2}{3 a g}, \quad m=\frac{128 e^{2 \delta}\left(3 e^{2 \delta}-1\right)^{3}}{729 g^{4}\left(e^{2 \delta}+1\right)^{2}\left(e^{2 \delta}-1\right)^{6}} .
$$

Hence, there is only one independent parameter in the BPS limit, which we choose to be $\delta$. In addition, all three roots of $T$, i.e. $r_{ \pm}$and $r_{0}$, coincide in the BPS limit, and its value is

$$
r_{*}^{2}=\frac{16}{3 g^{2}\left(3 e^{2 \delta}-5\right)\left(e^{2 \delta}+1\right)} .
$$

The thermodynamic quantities in the BPS limit become

$$
\begin{aligned}
T_{*} & =0, \\
S_{*} & =\frac{32 \pi^{3} \sqrt{9 e^{2 \delta}-7}}{3 \sqrt{3} G_{N} g^{5}\left(3 e^{2 \delta}-5\right)^{3}\left(e^{2 \delta}+1\right)^{3 / 2}}, \\
\Omega_{*} & =1, \\
\Phi_{*} & =2, \\
E_{*} & =\frac{16 \pi^{2}\left(18 e^{6 \delta}-21 e^{4 \delta}+7\right)}{3 G_{N} g^{5}\left(3 e^{2 \delta}-5\right)^{4}\left(e^{2 \delta}+1\right)^{2}}, \\
J_{*} & =\frac{16 \pi^{2}\left(9 e^{4 \delta}+18 e^{2 \delta}-23\right)}{9 G_{N} g^{5}\left(3 e^{2 \delta}-5\right)^{4}\left(e^{2 \delta}+1\right)^{2}}, \\
Q_{*} & =\frac{8 \pi^{2}\left(3 e^{6 \delta}-5 e^{4 \delta}-3 e^{2 \delta}+5\right)}{G_{N} g^{5}\left(3 e^{2 \delta}-5\right)^{4}\left(e^{2 \delta}+1\right)^{2}} .
\end{aligned}
$$




\subsubsection{Gravitational Cardy limit}

The Cardy-like limit for the $6 \mathrm{~d} \mathcal{N}=(2,0)$ theory was defined in [8], which for the special solution with three equal angular momenta is

$$
|\omega| \ll 1, \quad \Delta \sim \mathcal{O}(1) .
$$

Using the following relations found in [7]

$$
\omega=\frac{1}{T}\left(\Omega-\Omega_{*}\right), \quad \phi=\frac{1}{T}\left(\Phi-\Phi_{*}\right),
$$

we obtain the corresponding limit as

$$
\left|\left(\frac{\partial \Omega}{\partial T}\right)_{T=0}\right| \ll 1,\left.\quad \frac{\partial \Phi}{\partial T}\right|_{T=0} \sim \mathcal{O}(1) .
$$

Using the relation (4.13), we can express $\left(\frac{\partial \Omega}{\partial T}\right)_{*}$ in terms of the paramter $\delta$. The explicit form is not very elucidating, but we do find a root to the equation $\left(\frac{\partial \Omega}{\partial T}\right)_{*}=0$, which is

$$
\delta_{*}=\frac{1}{2} \log \left(\frac{5}{3}\right) .
$$

Hence, the gravitational Cardy limit for the class of asymptotically $\mathrm{AdS}_{7}$ BPS black holes (4.1) is

$$
\delta \rightarrow \frac{1}{2} \log \left(\frac{5}{3}\right)
$$

Note that this is equivalent to

$$
a g \rightarrow-1,
$$

as in the other black hole solutions. We can introduce a small parameter to denote the deviation from this limit, i.e.,

$$
\delta=\frac{1}{2} \log \left(\frac{5}{3}\right)+\epsilon .
$$

For this case $\epsilon$ is dimensionless. Expanding in $\epsilon$, we find the BPS thermodynamic quantities (4.11) in the gravitational Cardy limit (4.18) to the leading order

$$
\begin{aligned}
T_{*} & =0, \\
S_{*} & =\frac{\pi^{3}}{250 G_{N} g^{5} \epsilon^{3}}+\mathcal{O}\left(\epsilon^{-2}\right), \\
\Omega_{*} & =1, \\
\Phi_{*} & =2, \\
E_{*} & =\frac{3 \pi^{2}}{1250 G_{N} g^{5} \epsilon^{4}}+\mathcal{O}\left(\epsilon^{-3}\right), \\
J_{*} & =\frac{\pi^{2}}{1250 G_{N} g^{5} \epsilon^{4}}+\mathcal{O}\left(\epsilon^{-3}\right), \\
Q_{*} & =\frac{\pi^{2}}{500 G_{N} g^{5} \epsilon^{3}}+\mathcal{O}\left(\epsilon^{-2}\right),
\end{aligned}
$$

which are consistent with $[8,41]$ and the Cardy-like limit on the field theory side $(4.12)$

$$
\omega_{*} \sim \epsilon, \quad \Delta_{*} \sim \mathcal{O}(1) .
$$




\subsubsection{Black hole solution in the near-horizon + gravitational Cardy limit}

In the previous subsection, we have obtained the gravitational Cardy limit for the parameters on the gravity side. In this subsection, we discuss how the near-horizon metric changes when taking the gravitational Cardy limit. In appendix A.3, we verify explicitly that the resulting background is a solution of the $7 \mathrm{~d}$ gauged supergravity equations of motion. In the following, we implement the gravitational Cardy limit in the space of parameter which further simplifies the geometry.

We can apply the following scaling near the horizon $r_{*}$ (4.10) to the BPS $\mathrm{AdS}_{7}$ black hole metric (4.1)

$$
r \rightarrow r_{*}+\lambda \widetilde{r}, \quad t \rightarrow \frac{\tilde{t}}{\lambda}, \quad \chi \rightarrow \widetilde{\chi}-\frac{6 g \sinh (\delta)}{\cosh (\delta)+2 \sinh (\delta)} \frac{\tilde{t}}{\lambda},
$$

with $\lambda \rightarrow 0$. In addition, taking the gravitational Cardy limit (4.18), we obtain the nearhorizon metric to the leading order in $\epsilon$

$$
\begin{aligned}
d s^{2}= & -10 g^{2} 2^{2 / 5} \epsilon \widetilde{r}^{2} d \widetilde{t}^{2}+\frac{1}{16 g^{2} 2^{3 / 5}} \frac{d \widetilde{r}^{2}}{\widetilde{r}^{2}} \\
& +\frac{2^{2 / 5}}{25 g^{2} \epsilon^{2}}\left(d \widetilde{\chi}+\frac{1}{2} l_{3} \sin ^{2} \xi-5 \sqrt{5} g^{2} \epsilon^{3 / 2} \widetilde{r} d \widetilde{t}\right)^{2}+\frac{2^{2 / 5}}{5 g^{2} \epsilon} d s_{\mathbb{C P}^{2}}^{2} .
\end{aligned}
$$

Defining

$$
\tau \equiv 8 \sqrt{5} g^{2} \sqrt{\epsilon} \widetilde{t}
$$

we can rewrite the near-horizon metric in the gravitational Cardy limit (4.22) as follows

$$
d s^{2}=\frac{1}{16 g^{2} 2^{3 / 5}}\left[-\widetilde{r}^{2} d \tau^{2}+\frac{d \widetilde{r}^{2}}{\widetilde{r}^{2}}\right]+\frac{2^{2 / 5}}{25 g^{2} \epsilon^{2}}\left(d \widetilde{\chi}+\frac{1}{2} l_{3} \sin ^{2} \xi-\frac{5 \epsilon}{8} \widetilde{r} d \tau\right)^{2}+\frac{2^{2 / 5}}{5 g^{2} \epsilon} d s_{\mathbb{C P}^{2}}^{2} .
$$

\subsubsection{Black hole entropy from Cardy formula}

For the asymptotically $\mathrm{AdS}_{7}$ black holes discussed in this section, we apply the Cardy formula to the near-horizon metric only after taking the gravitational Cardy limit. More explicitly, we first rewrite the metric (4.24) from the Poincaré coordinates $(\widetilde{r}, \tau)$ to the global coordinates $(\hat{r}, \hat{t})$ using the relations $(2.44)-(2.46)$. Consequently, the near-horizon metric in the gravitational Cardy limit (4.24) becomes

$d s^{2}=\frac{1}{16 g^{2} 2^{3 / 5}}\left[-\left(1+\hat{r}^{2}\right) d \hat{t}^{2}+\frac{d \hat{r}^{2}}{1+\hat{r}^{2}}\right]+\frac{2^{2 / 5}}{25 g^{2} \epsilon^{2}}\left(d \hat{\chi}+\frac{1}{2} l_{3} \sin ^{2} \xi-\frac{5 \epsilon}{8} \hat{r} d \hat{t}\right)^{2}+\frac{2^{2 / 5}}{5 g^{2} \epsilon} d s_{\mathbb{C P}^{2}}^{2}$,

where $\hat{t}, \hat{r}$ and $\gamma$ are defined in (2.44) and (2.46), while

$$
\hat{\chi} \equiv \tilde{\chi}-\frac{5 \epsilon}{8} \gamma .
$$

Applying the same formalism in subsection 2.4, we obtain the central charge and the extremal Frolov-Thorne temperature in the near-horizon region of the asymptotically $\mathrm{AdS}_{7}$ BPS black holes as follows

$$
c_{L}=\frac{3 \pi^{2}}{200 g^{5} \epsilon^{2}}, \quad T_{L}=\frac{4}{5 \pi \epsilon} .
$$


Using the Cardy formula, we can compute the black hole entropy of the asymptotically $\mathrm{AdS}_{7}$ BPS black holes

$$
S_{B H}=\frac{\pi^{2}}{3} c_{L} T_{L}=\frac{\pi^{3}}{250 g^{5} \epsilon^{3}},
$$

which is the same as the black hole entropy in the gravitational Cardy limit (4.19) from the gravity side in the unit $G_{N}=1$.

\subsubsection{Comparison with results from boundary CFT}

The asymptotically $\mathrm{AdS}_{7}$ BPS black hole entropy can also be obtained from the boundary $6 \mathrm{~d}(2,0)$ theory by extremizing an entropy function $[4,7,8]$ originally motivated in [54] and more recently studied in [44]. We can first compute the free energy in the large- $N$ limit using the background field method on $S^{5}$, the partition function via localization or the $6 \mathrm{~d}$ superconformal index. The entropy function is then defined as a Legendre transform of the free energy in the large- $N$ limit

$$
S\left(\Delta_{I}, \omega_{i}\right)=-\frac{N^{3}}{24} \frac{\Delta_{1}^{2} \Delta_{2}^{2}}{\omega_{1} \omega_{2} \omega_{3}}+\sum_{I=1}^{2} Q_{I} \Delta_{I}+\sum_{i=1}^{3} J_{i} \omega_{i}-\Lambda\left(\sum_{I=1}^{2} \Delta_{I}-\sum_{i=1}^{3} \omega_{i}-2 \pi i\right) .
$$

In the Cardy-like limit (4.12)

$$
\omega \sim \epsilon, \quad \Delta_{I} \sim \mathcal{O}(1)
$$

we can read off from the entropy function (4.29)

$$
S \sim \frac{1}{\epsilon^{3}}, \quad J \sim \frac{1}{\epsilon^{4}}, \quad Q_{I} \sim \frac{1}{\epsilon^{3}},
$$

which have been summarized in table 1 .

Similar to $\mathrm{AdS}_{4,5}$, for $\mathrm{AdS}_{7}$ the electric charges $Q_{I}$ and the angular momenta $J_{i}$ are real, while the chemical potentials $\Delta_{I}$ and the angular velocities $\omega_{i}$ can be complex, and so can the entropy function $S$. By requiring that the black hole entropy $S_{B H}$ to be real after extremizing the entropy function $S$, we obtain one more constraint on $Q_{I}$ and $J_{i}$. More precisely, the most general case with two charges $\left(Q_{1}, Q_{2}\right)$ and three angular momenta $\left(J_{1}, J_{2}, J_{3}\right)$ was discussed in $[4,8]$, while the degenerate case with $Q_{1}=Q_{2}$ and $J_{1}=J_{2}=$ $J_{3}$ was discussed in [7]. For the most general case, the asymptotically $\mathrm{AdS}_{7}$ black hole entropy is $[4,8]$

$$
S_{B H}=2 \pi \sqrt{\frac{3\left(Q_{1}^{2} Q_{2}+Q_{1} Q_{2}^{2}\right)-N^{3}\left(J_{1} J_{2}+J_{2} J_{3}+J_{3} J_{1}\right)}{3\left(Q_{1}+Q_{2}\right)-N^{3}}},
$$

subject to the constraint

$$
\begin{aligned}
& \frac{3\left(Q_{1}^{2} Q_{2}+Q_{1} Q_{2}^{2}\right)-N^{3}\left(J_{1} J_{2}+J_{2} J_{3}+J_{3} J_{1}\right)}{3\left(Q_{1}+Q_{2}\right)-N^{3}} \\
= & {\left[\frac{N^{3}}{3}\left(J_{1}+J_{2}+J_{3}\right)+\frac{Q_{1}^{2}+Q_{2}^{2}}{2}+2 Q_{1} Q_{2}\right] } \\
& \times\left[1-\sqrt{1-\frac{\frac{2}{3} N^{3} J_{1} J_{2} J_{3}+Q_{1}^{2} Q_{2}^{2}}{\left(\frac{N^{3}}{3}\left(J_{1}+J_{2}+J_{3}\right)+\frac{Q_{1}^{2}+Q_{2}^{2}}{2}+2 Q_{1} Q_{2}\right)^{2}}}\right],
\end{aligned}
$$

which is a consequence of the reality condition on the black hole entropy $S_{B H}$. 
We apply the general result to the special case $Q_{1}=Q_{2}=Q$ and $J_{1}=J_{2}=J_{3}=J$,

$$
S_{B H}=2 \pi \sqrt{\frac{6 Q^{3}-3 N^{3} J^{2}}{6 Q-N^{3}}},
$$

with the constraint

$$
\frac{6 Q^{3}-3 N^{3} J^{2}}{6 Q-N^{3}}=\left(N^{3} J+3 Q^{2}\right) \cdot\left[1-\sqrt{1-\frac{\frac{2}{3} N^{3} J^{3}+Q^{4}}{N^{3} J+3 Q^{2}}}\right]
$$

which are consistent with both the thermodynamic quantities on the gravity side (4.11) (4.19) and the black hole entropy in the gravitational Cardy limit from the Cardy formula (4.28) under the $\mathrm{AdS}_{7} / \mathrm{CFT}_{6}$ dictionary of parameters $[4,8]$

$$
G_{N}=\frac{3 \pi^{2}}{16 g^{5} N^{3}}
$$

\subsection{More general case}

In the previous section, we have discussed a special solution of asymptotically $\mathrm{AdS}_{7}$ black holes with two equal charges $Q_{1}=Q_{2}$ and three equal angular momenta $J_{1}=J_{2}=J_{3}$. In this subsection, we consider a more general solution with two equal charges $Q_{1}=Q_{2}$ and three independent angular momenta $\left(J_{1}, J_{2}, J_{3}\right)$, which was first introduced in [52].

\subsection{1 $\quad \mathrm{AdS}_{7}$ black hole solution}

The metric for this class of asymptotically $\mathrm{AdS}_{7}$ black holes is

$$
\begin{aligned}
d s^{2}= & H^{2 / 5}\left[\frac{\left(r^{2}+y^{2}\right)\left(r^{2}+z^{2}\right)}{R} d r^{2}+\frac{\left(r^{2}+y^{2}\right)\left(y^{2}-z^{2}\right)}{Y} d y^{2}+\frac{\left(r^{2}+z^{2}\right)\left(z^{2}-y^{2}\right)}{Z} d z^{2}\right. \\
& -\frac{R}{H^{2}\left(r^{2}+y^{2}\right)\left(r^{2}+z^{2}\right)} \mathcal{A}^{2} \\
& +\frac{Y}{\left(r^{2}+y^{2}\right)\left(y^{2}-z^{2}\right)}\left(d t^{\prime}+\left(z^{2}-r^{2}\right) d \psi_{1}-r^{2} z^{2} d \psi_{2}-\frac{q}{H\left(r^{2}+y^{2}\right)\left(r^{2}+z^{2}\right)} \mathcal{A}\right)^{2} \\
& +\frac{Z}{\left(r^{2}+z^{2}\right)\left(z^{2}-y^{2}\right)}\left(d t^{\prime}+\left(y^{2}-r^{2}\right) d \psi_{1}-r^{2} y^{2} d \psi_{2}-\frac{q}{H\left(r^{2}+y^{2}\right)\left(r^{2}+z^{2}\right)} \mathcal{A}\right)^{2} \\
& +\frac{a_{1}^{2} a_{2}^{2} a_{3}^{2}}{r^{2} y^{2} z^{2}}\left(d t^{\prime}+\left(y^{2}+z^{2}-r^{2}\right) d \psi_{1}+\left(y^{2} z^{2}-r^{2} y^{2}-r^{2} z^{2}\right) d \psi_{2}-r^{2} y^{2} z^{2} d \psi_{3}\right. \\
& \left.\left.-\frac{q}{H\left(r^{2}+y^{2}\right)\left(r^{2}+z^{2}\right)}\left(1+\frac{g y^{2} z^{2}}{a_{1} a_{2} a_{3}}\right) \mathcal{A}\right)^{2}\right]
\end{aligned}
$$


while the 1 -form, the 2-form, the 3 -form and the scalar are

$$
\begin{aligned}
A_{(1)}= & \frac{2 m \sinh (\delta) \cosh (\delta)}{H\left(r^{2}+y^{2}\right)\left(r^{2}+z^{2}\right)} \mathcal{A}, \\
A_{(2)}= & \frac{q}{H\left(r^{2}+y^{2}\right)\left(r^{2}+z^{2}\right)} \mathcal{A} \wedge \\
& \quad\left[d t^{\prime}+\sum_{i} a_{i}^{2}\left(g^{2} d t^{\prime}+d \psi_{1}+\sum_{i<j} a_{i}^{2} a_{j}^{2}\left(g^{2} d \psi_{1}+d \psi_{2}\right)+a_{1}^{2} a_{2}^{2} a_{3}^{2}\left(g^{2} d \psi_{2}+d \psi_{3}\right)\right.\right. \\
& \left.\quad-g^{2}\left(y^{2}+z^{2}\right) d t^{\prime}-g^{2} y^{2} z^{2} d \psi_{1}+a_{1} a_{2} a_{3}\left(d \psi_{1}+\left(y^{2}+z^{2}\right) d \psi_{2}+y^{2} z^{2} d \psi_{3}\right)\right], \\
A_{(3)}= & q a_{1} a_{2} a_{3}\left[d \psi_{1}+\left(y^{2}+z^{2}\right) d \psi_{2}+y^{2} z^{2} d \psi_{3}\right] \\
& \wedge\left(\frac{1}{\left(r^{2}+y^{2}\right) z} d z \wedge\left(d \psi_{1}+y^{2} d \psi_{2}\right)+\frac{1}{\left(r^{2}+z^{2}\right) y} d y \wedge\left(d \psi_{1}+z^{2} d \psi_{2}\right)\right) \\
& -q g \mathcal{A} \wedge\left(\frac{z}{r^{2}+y^{2}} d z \wedge\left(d \psi_{1}+y^{2} d \psi_{2}\right)+\frac{y}{r^{2}+z^{2}} d y \wedge\left(d \psi_{1}+z^{2} d \psi_{2}\right)\right), \\
X= & H^{-1 / 5},
\end{aligned}
$$

where

$$
\begin{aligned}
R & \equiv \frac{1+g^{2} r^{2}}{r^{2}} \prod_{i=1}^{3}\left(r^{2}+a_{i}^{2}\right)+q g^{2}\left(2 r^{2}+a_{1}^{2}+a_{2}^{2}+a_{3}^{2}\right)-\frac{2 q g a_{1} a_{2} a_{3}}{r^{2}}+\frac{q^{2} g^{2}}{r^{2}}-2 m, \\
Y & \equiv \frac{1-g^{2} y^{2}}{y^{2}} \prod_{i=1}^{3}\left(a_{i}^{2}-y^{2}\right), \\
Z & \equiv \frac{1-g^{2} z^{2}}{z^{2}} \prod_{i=1}^{3}\left(a_{i}^{2}-z^{2}\right), \\
\mathcal{A} & \equiv d t^{\prime}+\left(y^{2}+z^{2}\right) d \psi_{1}+y^{2} z^{2} d \psi_{2}, \\
H & \equiv 1+\frac{q}{\left(r^{2}+y^{2}\right)\left(r^{2}+z^{2}\right)}, \\
q & \equiv 2 m \sinh ^{2}(\delta) .
\end{aligned}
$$

It can be shown that after the change of coordinates

$$
\begin{aligned}
t & =t^{\prime}+\left(a_{1}^{2}+a_{2}^{2}+a_{3}^{2}\right) \psi_{1}+\left(a_{1}^{2} a_{2}^{2}+a_{2}^{2} a_{3}^{2}+a_{3}^{2} a_{1}^{2}\right) \psi_{2}+a_{1}^{2} a_{2}^{2} a_{3}^{2} \psi_{3}, \\
\frac{\phi_{i}}{a_{i}} & =g^{2} t^{\prime}+\psi_{1}+\sum_{j \neq i} a_{j}^{2}\left(g^{2} \psi_{1}+\psi_{2}\right)+\prod_{j \neq i} a_{j}^{2}\left(g^{2} \psi_{2}+\psi_{3}\right),
\end{aligned}
$$


the metric (4.37) can be written in an equivalent form

$$
\begin{aligned}
d s^{2}= & H^{2 / 5}\left[\frac{\left(r^{2}+y^{2}\right)\left(r^{2}+z^{2}\right)}{R} d r^{2}+\frac{\left(r^{2}+y^{2}\right)\left(y^{2}-z^{2}\right)}{Y} d y^{2}+\frac{\left(r^{2}+z^{2}\right)\left(z^{2}-y^{2}\right)}{Z} d z^{2}\right. \\
& -\frac{r^{2} y^{2} z^{2} R Y Z}{H^{2} \prod_{i<j}\left(a_{i}^{2}-a_{j}^{2}\right)^{2} B_{1} B_{2} B_{3}} d t^{2}+B_{3}\left(d \phi_{3}+v_{32} d \phi_{2}+v_{31} d \phi_{1}+v_{30} d t\right)^{2} \\
& \left.+B_{2}\left(d \phi_{2}+v_{21} d \phi_{1}+v_{20} d t\right)^{2}+B_{1}\left(d \phi_{1}+v_{10} d t\right)^{2}\right]
\end{aligned}
$$

where $B_{1}, B_{2}, B_{3}, v_{10}, v_{20}, v_{21}, v_{30}, v_{31}$ and $v_{32}$ can be determined by comparing (4.41) with (4.37). We can see that in the gravitational Cardy limit $B_{1}$ and $B_{2}$ are subleading compared to $B_{3}$. Hence, qualitatively the term $B_{3}\left(d \phi_{3}+v_{32} d \phi_{2}+v_{31} d \phi_{1}+v_{30} d t\right)^{2}$ in the metric forms the only $\mathrm{U}(1)$ circle fibered over $\mathrm{AdS}_{2}$ in the gravitational Cardy limit of the near-horizon solution, similar to the other cases in this paper. However, because the explicit expressions of these coefficients are lengthy and not very illuminating, we do not list them here.

The thermodynamic quantities can be expressed as

$$
\begin{aligned}
E & =\frac{\pi^{2}}{8 \Xi_{1} \Xi_{2} \Xi_{3}}\left[\sum_{i} \frac{2 m}{\Xi_{i}}-m+\frac{5 q}{2}+\frac{q}{2} \sum_{i}\left(\sum_{j \neq i} \frac{2 \Xi_{j}}{\Xi_{i}}-\Xi_{i}-\frac{2\left(1+2 a_{1} a_{2} a_{3} g^{3}\right)}{\Xi_{i}}\right)\right], \\
T & =\frac{\left(1+g^{2} r_{+}^{2}\right) r_{+}^{2} \sum_{i} \prod_{j \neq i}\left(r_{+}^{2}+a_{j}^{2}\right)-\prod_{i}\left(r_{+}^{2}+a_{i}^{2}\right)+2 q\left(g^{2} r_{+}^{4}+g a_{1} a_{2} a_{3}\right)-q^{2} g^{2}}{2 \pi r_{+}\left[\left(r_{+}^{2}+a_{1}^{2}\right)\left(r_{+}^{2}+a_{2}^{2}\right)\left(r_{+}^{2}+a_{3}^{2}\right)+q\left(r_{+}^{2}-a_{1} a_{2} a_{3} g\right)\right]} \\
S & =\frac{\pi^{3}\left[\left(r_{+}^{2}+a_{1}^{2}\right)\left(r_{+}^{2}+a_{2}^{2}\right)\left(r_{+}^{2}+a_{3}^{2}\right)+q\left(r_{+}^{2}-a_{1} a_{2} a_{3} g\right)\right]}{4 \Xi_{1} \Xi_{2} \Xi_{3} r_{+}}, \\
\Omega_{i} & =\frac{a_{i}\left[\left(1+g^{2} r_{+}^{2}\right) \prod_{j \neq i}\left(r_{+}^{2}+a_{j}^{2}\right)+q g^{2} r_{+}^{2}\right]-q \prod_{j \neq i} a_{j} g}{\left(r_{+}^{2}+a_{1}^{2}\right)\left(r_{+}^{2}+a_{2}^{2}\right)\left(r_{+}^{2}+a_{3}^{2}\right)+q\left(r_{+}^{2}-a_{1} a_{2} a_{3} g\right)}, \\
J_{i} & =\frac{\pi^{2} m\left[a_{i} \cosh ^{2}(\delta)-g \sinh ^{2}(\delta)\left(\prod_{j \neq i} a_{j}+a_{i} \sum_{j \neq i} a_{j}^{2} g+a_{1} a_{2} a_{3} a_{i} g^{2}\right)\right]}{4 \Xi_{1} \Xi_{2} \Xi_{3} \Xi_{i}}, \\
\Phi & =\frac{2 m \sinh (\delta) \cosh (\delta) r_{+}^{2}}{\left(r_{+}^{2}+a_{1}^{2}\right)\left(r_{+}^{2}+a_{2}^{2}\right)\left(r_{+}^{2}+a_{3}^{2}\right)+q\left(r_{+}^{2}-a_{1} a_{2} a_{3} g\right)}, \\
Q & =\frac{\pi^{2} m \sinh (\delta) \cosh (\delta)}{\Xi_{1} \Xi_{2} \Xi_{3}},
\end{aligned}
$$

where $r_{+}$denotes the position of the outer horizon, and

$$
\Xi_{i} \equiv 1-a_{i}^{2} g^{2}, \quad \Xi_{i \pm} \equiv 1 \pm a_{i} g, \quad(i=1,2,3) .
$$

This class of asymptotically $\mathrm{AdS}_{7}$ black hole solutions is characterized by five parameters $\left(m, \delta, a_{1}, a_{2}, a_{3}\right)$. The BPS limit for this class of solutions is

$$
e^{2 \delta}=1-\frac{2}{\left(a_{1}+a_{2}+a_{3}\right) g},
$$


while the naked closed timelike curves (CTCs) can be avoided by requiring an additional condition

$$
q=-\frac{\Xi_{1-} \Xi_{2-} \Xi_{3-}\left(a_{1}+a_{2}\right)\left(a_{2}+a_{3}\right)\left(a_{3}+a_{1}\right)}{\left(1-a_{1} g-a_{2} g-a_{3} g\right)^{2} g} .
$$

Hence, only three parameters are independent, which we can choose to be $\left(a_{1}, a_{2}, a_{3}\right)$. In the BPS limit, the thermodynamic quantities can be simplified as follows

$$
\begin{aligned}
& E=-\frac{\pi^{2} \prod_{k<l}\left(a_{k}+a_{l}\right)\left[\sum_{i} \Xi_{i}+\sum_{i<j} \Xi_{i} \Xi_{j}-\left(1+a_{1} a_{2} a_{3} g^{3}\right)\left(2+\sum_{i} a_{i} g+\sum_{i<j} a_{i} a_{j} g^{2}\right)\right]}{8 \Xi_{1+}^{2} \Xi_{2+}^{2} \Xi_{3+}^{2}\left(1-a_{1} g-a_{2} g-a_{3} g\right)^{2} g}, \\
& T=0, \quad \Omega_{i}=-g, \quad \Phi=1, \\
& S=-\frac{\pi^{3}\left(a_{1}+a_{2}\right)\left(a_{2}+a_{3}\right)\left(a_{3}+a_{1}\right)\left(a_{1} a_{2}+a_{2} a_{3}+a_{3} a_{1}-a_{1} a_{2} a_{3} g\right)}{4 \Xi_{1+} \Xi_{2+} \Xi_{3+}\left(1-a_{1} g-a_{2} g-a_{3} g\right)^{2} g r_{0}}, \\
& J_{i}=-\frac{\pi^{2}\left(a_{1}+a_{2}\right)\left(a_{2}+a_{3}\right)\left(a_{3}+a_{1}\right)\left[a_{i}-\left(a_{i}^{2}+2 a_{i} \sum_{j \neq i} a_{j}+\prod_{j \neq i} a_{j}\right) g+a_{1} a_{2} a_{3} g^{2}\right]}{8 \Xi_{1+} \Xi_{2+} \Xi_{3+} \Xi_{i+}\left(1-a_{1} g-a_{2} g-a_{3} g\right)^{2} g}, \\
& Q=-\frac{\pi^{2}\left(a_{1}+a_{2}\right)\left(a_{2}+a_{3}\right)\left(a_{3}+a_{1}\right)}{2 \Xi_{1+} \Xi_{2+} \Xi_{3+}\left(1-a_{1} g-a_{2} g-a_{3} g\right) g},
\end{aligned}
$$

where

$$
r_{0}=\sqrt{\frac{a_{1} a_{2}+a_{2} a_{3}+a_{3} a_{1}-a_{1} a_{2} a_{3} g}{1-a_{1} g-a_{2} g-a_{3} g}} .
$$

\subsubsection{Gravitational Cardy limit}

Similar to subsection 4.1.2, for the more general $\mathrm{AdS}_{7}$ solution with three independent angular momenta, we can translate the Cardy limit for the $6 \mathrm{~d} \mathcal{N}=(2,0)$ theory defined in $[8]$

$$
\left|\omega_{i}\right| \ll 1, \quad \Delta \sim \mathcal{O}(1), \quad(i=1,2,3)
$$

into the gravitational Cardy-like limit for this class of asymptotically $\mathrm{AdS}_{7}$ black holes

$$
\left|\left(\frac{\partial \Omega_{i}}{\partial T}\right)_{T=0}\right| \ll 1,\left.\quad \frac{\partial \Phi}{\partial T}\right|_{T=0} \sim \mathcal{O}(1) .
$$

A choice of the parameters $\left(a_{1}, a_{2}, a_{3}\right)$ that satisfies the limit (4.49) is

$$
a_{1}=a_{2}=a_{3}=-\frac{1}{g}
$$

As in the other black hole solutions, this can be summarized as

$$
a_{i} g \rightarrow-1
$$

We can introduce a small parameter $\epsilon$ to denote the deviation from this limit, i.e.,

$$
a_{i}=-\frac{1}{g}+\epsilon, \quad(i=1,2,3),
$$


or in a more refined way

$$
a_{1}=-\frac{1}{g}+\epsilon, \quad a_{2}=-\frac{1}{g}+\epsilon+\eta_{1}, \quad a_{3}=-\frac{1}{g}+\epsilon+\eta_{2}, \quad\left(\eta_{1}, \eta_{2} \ll \epsilon\right) .
$$

Expanding in $\epsilon$, after expanding in $\eta_{1}$ and $\eta_{2}$, we find the BPS thermodynamic quantities (4.46) in the gravitational Cardy limit (4.52) to the leading order

$$
\begin{aligned}
S_{*} & =\frac{\pi^{3}}{2 g^{8} \epsilon^{3}}+\mathcal{O}\left(\epsilon^{-2}\right), \\
J_{i}^{*} & =-\frac{\pi^{2}}{2 g^{9} \epsilon^{4}}+\mathcal{O}\left(\epsilon^{-3}\right), \\
Q_{*} & =\frac{\pi^{2}}{g^{7} \epsilon^{3}}+\mathcal{O}\left(\epsilon^{-2}\right),
\end{aligned}
$$

which are consistent with $[8,41]$ and the Cardy-like limit on the field theory side (4.48)

$$
\omega_{i *} \sim \epsilon, \quad \Delta_{*} \sim \mathcal{O}(1)
$$

\subsubsection{Black hole solution in the near-horizon limit}

In this subsection, we consider the near-horizon limit of the asymptotically $\mathrm{AdS}_{7}$ black hole metric. As mentioned in subsection 4.2.1, we should in principle take the Cardy limit of the near horizon solution (4.41). Applying the refined gravitational Cardy limit (4.53), we find that $B_{1}$ and $B_{2}$ are subleading compared to $B_{3}$. Therefore, in the near-horizon limit we obtain an $\mathrm{AdS}_{3}$ geometry, just like the other cases. However, in practice the expressions of the coefficients are lengthy, so we consider an alternative near-horizon metric discussed in [20]. That is, the metric (4.37) can be expressed in an equivalent form

$$
\begin{aligned}
d s^{2}=H^{2 / 5}[ & \frac{\left(r^{2}+y^{2}\right)\left(r^{2}+z^{2}\right)}{R} d r^{2}+\frac{\left(r^{2}+y^{2}\right)\left(y^{2}-z^{2}\right)}{Y} d y^{2}+\frac{\left(r^{2}+z^{2}\right)\left(z^{2}-y^{2}\right)}{Z} d z^{2} \\
& +\frac{Y}{\left(r^{2}+y^{2}\right)\left(y^{2}-z^{2}\right)}\left(d t-\sum_{i=1}^{3} \frac{\left(r^{2}+a_{i}^{2}\right) \gamma_{i}}{a_{i}^{2}-y^{2}} \frac{d \hat{\phi}_{i}}{\delta_{i}}-\frac{q \mathcal{A}}{H\left(r^{2}+y^{2}\right)\left(r^{2}+z^{2}\right)}\right)^{2} \\
& +\frac{Z}{\left(r^{2}+z^{2}\right)\left(z^{2}-y^{2}\right)}\left(d t-\sum_{i=1}^{3} \frac{\left(r^{2}+a_{i}^{2}\right) \gamma_{i}}{a_{i}^{2}-z^{2}} \frac{d \hat{\phi}_{i}}{\delta_{i}}-\frac{q \mathcal{A}}{H\left(r^{2}+y^{2}\right)\left(r^{2}+z^{2}\right)}\right)^{2} \\
& \left.+\frac{a_{1}^{2} a_{2}^{2} a_{3}^{2}}{r^{2} y^{2} z^{2}}\left(d t-\sum_{i=1}^{3} \frac{\left(r^{2}+a_{i}^{2}\right) \gamma_{i}}{a_{i}^{2}} \frac{d \hat{\phi}_{i}}{\delta_{i}}-\frac{q \mathcal{A}}{H\left(r^{2}+y^{2}\right)\left(r^{2}+z^{2}\right)}\left(1+\frac{g y^{2} z^{2}}{a_{1} a_{2} a_{3}}\right)\right)^{2}\right],
\end{aligned}
$$

where we have used the changes of coordinates (4.40) and

$$
\begin{aligned}
\hat{\phi}_{i} & \equiv \phi_{i}-a_{i} g^{2} t, \quad(i=1,2,3), \\
\gamma_{i} & \equiv a_{i}^{2}\left(a_{i}^{2}-y^{2}\right)\left(a_{i}^{2}-z^{2}\right), \\
\delta_{i} & \equiv a_{i}\left(1-a_{i}^{2} g^{2}\right) \prod_{j \neq i}\left(a_{i}^{2}-a_{j}^{2}\right) .
\end{aligned}
$$


Applying the following scaling to the new metric (4.56)

$$
r \rightarrow r_{0}(1+\lambda \rho), \quad \phi \rightarrow \widetilde{\phi}_{i}+\frac{\Omega_{i}^{0}}{2 \pi T_{H}^{\prime 0} r_{0} \lambda} \widetilde{t}, \quad t \rightarrow \frac{\widetilde{t}}{2 \pi T_{H}^{\prime 0} r_{0} \lambda},
$$

which is slightly different from the original Bardeen-Horowitz scaling [21], we obtain the near-horizon geometry in the limit $\lambda \rightarrow 0$

$$
\begin{aligned}
d s^{2}= & H_{0}^{2 / 5}\left[\frac{U_{0}}{V}\left(-\rho^{2} d \widetilde{t}^{2}+\frac{d \rho^{2}}{\rho^{2}}\right)+\frac{\left(r_{0}^{2}+y^{2}\right)\left(y^{2}-z^{2}\right)}{Y} d y^{2}+\frac{\left(r_{0}^{2}+z^{2}\right)\left(z^{2}-y^{2}\right)}{Z} d z^{2}\right. \\
& +\frac{Y}{\left(r_{0}^{2}+y^{2}\right)\left(y^{2}-z^{2}\right)}\left(\frac{2 r_{0}\left(r_{0}^{2}+z^{2}\right)}{V} \rho d \widetilde{t}+\sum_{i=1}^{3} \frac{\left(r_{0}^{2}+a_{i}^{2}\right) \gamma_{i}}{a_{i}^{2}-y^{2}} \frac{d \widetilde{\phi}_{i}}{\delta_{i}}+\frac{q \widetilde{A}}{H_{0} U_{0}}\right)^{2} \\
& +\frac{Z}{\left(r_{0}^{2}+z^{2}\right)\left(z^{2}-y^{2}\right)}\left(\frac{2 r_{0}\left(r_{0}^{2}+y^{2}\right)}{V} \rho d \widetilde{t}+\sum_{i=1}^{3} \frac{\left(r_{0}^{2}+a_{i}^{2}\right) \gamma_{i}}{a_{i}^{2}-z^{2}} \frac{d \widetilde{\phi}_{i}}{\delta_{i}}+\frac{q \widetilde{A}}{H_{0} U_{0}}\right)^{2} \\
& \left.+\frac{a_{1}^{2} a_{2}^{2} a_{3}^{2}}{r_{0}^{2} y^{2} z^{2}}\left(\frac{2}{V r_{0}}\left(U_{0}-\frac{q g y^{2} z^{2}}{a_{1} a_{2} a_{3}}\right) \rho d \widetilde{t}+\sum_{i=1}^{3} \frac{\left(r_{0}^{2}+a_{i}^{2}\right) \gamma_{i}}{a_{i}^{2}} \frac{d \widetilde{\phi}_{i}}{\delta_{i}}+\frac{q \widetilde{A}}{H_{0} U_{0}}\left(1+\frac{g y^{2} z^{2}}{a_{1} a_{2} a_{3}}\right)\right)^{2}\right]
\end{aligned}
$$

where

$$
\begin{aligned}
U & \equiv\left(r^{2}+y^{2}\right)\left(r^{2}+z^{2}\right) \\
\gamma_{i} & \equiv a_{i}^{2}\left(a_{i}^{2}-y^{2}\right)\left(a_{i}^{2}-z^{2}\right) \\
\delta_{i} & \equiv \Xi_{i} a_{i} \prod_{j \neq i}\left(a_{i}^{2}-a_{j}^{2}\right) \\
U_{0} & \left.\equiv U\right|_{r=r_{0}}=\left(r_{0}^{2}+y^{2}\right)\left(r_{0}^{2}+z^{2}\right) \\
H_{0} & \left.\equiv H\right|_{r=r_{0}}=1+\frac{q}{\left(r_{0}^{2}+y^{2}\right)\left(r_{0}^{2}+z^{2}\right)} \\
V & \equiv 6 r_{0}^{2}+\sum_{i=1}^{3} a_{i}^{2}+\frac{3\left(a_{1} a_{2} a_{3}-q g\right)^{2}}{r_{0}^{4}}+g^{2}\left[15 r_{0}^{4}+6 r_{0}^{2} \sum_{i=1}^{3} a_{i}^{2}+\sum_{1 \leq i<j \leq 3} a_{i}^{2} a_{j}^{2}+2 q\right] \\
\widetilde{A} & \equiv-\frac{2 r_{0}\left(2 r_{0}^{2}+y^{2}+z^{2}\right)}{V} \rho d \widetilde{t}-\sum_{i=1}^{3} \gamma_{i} \frac{d \widetilde{\phi}_{i}}{\delta_{i}}
\end{aligned}
$$

Taking the refined gravitational Cardy limit (4.53), we can see that two of the three $\mathrm{U}(1)$ circles in the near-horizon metric (4.59) become degenerate. However, the remaining two $\mathrm{U}(1)$ circles are still of the same order in the gravitational Cardy limit. This is expected, because we should take the gravitational Cardy limit of the near-horizon of the metric (4.41) instead of (4.56), in order to have only one $\mathrm{U}(1)$ circle fibered over $\mathrm{AdS}_{2}$ in the nearhorizon plus gravitational Cardy limit. Nevertheless, the gravitational Cardy limit reduces some redundant $\mathrm{U}(1)$ circles, while keeping the essential information for the near-horizon Virasoro algebra. 


\subsubsection{Black hole entropy from Cardy formula}

We can apply the formalism described in subsection 2.4. The central charge and the extremal Frolov-Thorne temperature in the near-horizon region of the asymptotically $\mathrm{AdS}_{7}$ BPS black holes (4.59) were obtained in [20]. In the refined gravitational Cardy limit (4.53), the results are

$$
c_{L}=\frac{48 \pi^{2}}{g^{9} \epsilon^{2} V}, \quad T_{L}=\frac{g V}{32 \pi \epsilon} .
$$

Hence, the black hole entropy from the Cardy formula in the gravitational Cardy limit is

$$
S_{B H}=\frac{\pi^{2}}{3} c_{L} T_{L}=\frac{\pi^{3}}{2 g^{8} \epsilon^{3}},
$$

which is exactly the same as the result from the gravity solution (4.54).

\subsubsection{Comparison with results from boundary CFT}

As we discussed in subsection 4.1.5, for the asymptotically $\mathrm{AdS}_{7}$ black holes with general charges $\left(Q_{1}, Q_{2}\right)$ and angular momenta $\left(J_{1}, J_{2}, J_{3}\right)$, the entropy can be obtained from the boundary $6 \mathrm{~d}(2,0)$ theory $[4,8]$, and the results are summarized in (4.32) subject to the constraint (4.33).

We have discussed a degenerate case in subsection 4.1 with $Q_{1}=Q_{2}$ and $J_{1}=J_{2}=$ $J_{3}$. In this subsection, we have seen another degenerate case with $Q_{1}=Q_{2}=Q$ and $\left(J_{1}, J_{2}, J_{3}\right)$, which consequently has the black hole entropy

$$
S_{B H}=2 \pi \sqrt{\frac{6 Q^{3}-N^{3}\left(J_{1} J_{2}+J_{2} J_{3}+J_{3} J_{1}\right)}{6 Q-N^{3}}},
$$

subject to the constraint

$$
\begin{aligned}
& \frac{6 Q^{3}-N^{3}\left(J_{1} J_{2}+J_{2} J_{3}+J_{3} J_{1}\right)}{6 Q-N^{3}} \\
& =\left[\frac{N^{3}}{3}\left(J_{1}+J_{2}+J_{3}\right)+3 Q^{2}\right] \cdot\left[1-\sqrt{1-\frac{\frac{2}{3} N^{3} J_{1} J_{2} J_{3}+Q^{4}}{\left(\frac{N^{3}}{3}\left(J_{1}+J_{2}+J_{3}\right)+3 Q^{2}\right)^{2}}}\right],
\end{aligned}
$$

which are consistent with both the thermodynamic quantities on the gravity side (4.46) (4.54) and the black hole entropy in the gravitational Cardy limit from the Cardy formula (4.62) under the $\mathrm{AdS}_{7} / \mathrm{CFT}_{6}$ dictionary of parameters $[4,8]$

$$
G_{N}=\frac{3 \pi^{2}}{16 g^{5} N^{3}} .
$$

\section{Asymptotically $\mathrm{AdS}_{6}$ black holes}

In this section, we consider the asymptotically $\mathrm{AdS}_{6}$ black holes and the corresponding gravitational Cardy limit. Similar to the other cases, we demonstrate that the AdS 6 black hole entropy can be computed in various ways as shown in figure 1, and the other thermodynamic quantities scale correspondingly in the gravitational Cardy limit. 


\section{1 $\quad$ AdS $_{6}$ black hole solution}

In this subsection, we discuss the near-horizon plus Cardy limit of the non-extremal asymptotically $\mathrm{AdS}_{6}$ black holes constructed in [55], which are solutions to $6 \mathrm{~d} \mathcal{N}=4 \mathrm{SU}(2)$ gauged supergravity.

The bosonic part of this class of solution is given by the metric, a scalar, a 1-form potential and a 2 -form potential. The metric is

$$
\begin{aligned}
d s^{2}= & H^{1 / 2}\left[\frac{\left(r^{2}+y^{2}\right)\left(r^{2}+z^{2}\right)}{R} d r^{2}+\frac{\left(r^{2}+y^{2}\right)\left(y^{2}-z^{2}\right)}{Y} d y^{2}+\frac{\left(r^{2}+z^{2}\right)\left(z^{2}-y^{2}\right)}{Z} d z^{2}\right. \\
& -\frac{R}{H^{2}\left(r^{2}+y^{2}\right)\left(r^{2}+z^{2}\right)} \mathcal{A}^{2} \\
& +\frac{Y}{\left(r^{2}+y^{2}\right)\left(y^{2}-z^{2}\right)}\left(d t^{\prime}+\left(z^{2}-r^{2}\right) d \psi_{1}-r^{2} z^{2} d \psi_{2}-\frac{q r \mathcal{A}}{H\left(r^{2}+y^{2}\right)\left(r^{2}+z^{2}\right)}\right)^{2} \\
& \left.+\frac{Z}{\left(r^{2}+z^{2}\right)\left(z^{2}-y^{2}\right)}\left(d t^{\prime}+\left(y^{2}-r^{2}\right) d \psi_{1}-r^{2} y^{2} d \psi_{2}-\frac{q r \mathcal{A}}{H\left(r^{2}+y^{2}\right)\left(r^{2}+z^{2}\right)}\right)^{2}\right],
\end{aligned}
$$

while the 1-form potential, the 2-form potential and the scalar are

$$
\begin{aligned}
& A_{(1)}=\frac{2 m r \sinh (\delta) \cosh (\delta)}{H\left(r^{2}+y^{2}\right)\left(r^{2}+z^{2}\right)} \mathcal{A}+\frac{\alpha_{6}}{d t} \\
& \begin{aligned}
A_{(2)}=\frac{q}{H\left(r^{2}+y^{2}\right)^{2}\left(r^{2}+z^{2}\right)^{2}}\left[-\frac{y z\left(2 r\left(2 r^{2}+y^{2}+z^{2}\right)+q\right)}{H} d r \wedge \mathcal{A}\right. \\
+z\left(\left(r^{2}+z^{2}\right)\left(r^{2}-y^{2}\right)+q r\right) d y
\end{aligned} \\
& \quad \wedge\left(d t^{\prime}+\left(z^{2}-r^{2}\right) d \psi_{1}-r^{2} z^{2} d \psi_{2}-\frac{q r \mathcal{A}}{H\left(r^{2}+y^{2}\right)\left(r^{2}+z^{2}\right)}\right) \\
& +y\left(\left(r^{2}+y^{2}\right)\left(r^{2}-z^{2}\right)+q r\right) d z \\
& \left.\quad \wedge\left(d t^{\prime}+\left(y^{2}-r^{2}\right) d \psi_{1}-r^{2} y^{2} d \psi_{2}-\frac{q r \mathcal{A}}{H\left(r^{2}+y^{2}\right)\left(r^{2}+z^{2}\right)}\right)\right]
\end{aligned}
$$

where

$$
\begin{aligned}
t^{\prime} & \equiv \frac{t}{\Xi_{a} \Xi_{b}}-\frac{a^{4} \phi_{1}}{\Xi_{a} a\left(a^{2}-b^{2}\right)}-\frac{b^{4} \phi_{2}}{\Xi_{b} b\left(b^{2}-a^{2}\right)}, \\
\psi_{1} & \equiv-\frac{g^{2} t}{\Xi_{a} \Xi_{b}}+\frac{a^{2} \phi_{1}}{\Xi_{a} a\left(a^{2}-b^{2}\right)}+\frac{b^{2} \phi_{2}}{\Xi_{b} b\left(b^{2}-a^{2}\right)}, \\
\psi_{2} & \equiv \frac{g^{4} t}{\Xi_{a} \Xi_{b}}-\frac{\phi_{1}}{\Xi_{a} a\left(a^{2}-b^{2}\right)}-\frac{\phi_{2}}{\Xi_{b} b\left(b^{2}-a^{2}\right)},
\end{aligned}
$$


and

$$
\begin{aligned}
R & \equiv\left(r^{2}+a^{2}\right)\left(r^{2}+b^{2}\right)+g^{2}\left[r\left(r^{2}+a^{2}\right)+q\right]\left[r\left(r^{2}+b^{2}\right)+q\right]-2 m r, \\
Y & \equiv-\left(1-g^{2} y^{2}\right)\left(a^{2}-y^{2}\right)\left(b^{2}-y^{2}\right), \\
Z & \equiv-\left(1-g^{2} z^{2}\right)\left(a^{2}-z^{2}\right)\left(b^{2}-z^{2}\right), \\
H & \equiv 1+\frac{q r}{\left(r^{2}+y^{2}\right)\left(r^{2}+z^{2}\right)}, \\
\mathcal{A} & \equiv d t^{\prime}+\left(y^{2}+z^{2}\right) d \psi_{1}+y^{2} z^{2} d \psi_{2}, \\
q & \equiv 2 m \sinh ^{2}(\delta), \quad \Xi_{a} \equiv 1-a^{2} g^{2}, \quad \Xi_{b} \equiv 1-b^{2} g^{2} .
\end{aligned}
$$

Note that we have added a pure gauge term to the 1-form potential. It was shown in [55] that the metric (5.1) can be written in an equivalent form

$$
\begin{aligned}
d s^{2}=H^{1 / 2} & {\left[\frac{\left(r^{2}+y^{2}\right)\left(r^{2}+z^{2}\right)}{R} d r^{2}+\frac{\left(r^{2}+y^{2}\right)\left(y^{2}-z^{2}\right)}{Y} d y^{2}+\frac{\left(r^{2}+z^{2}\right)\left(z^{2}-y^{2}\right)}{Z} d z^{2}\right.} \\
& +\frac{R Y Z}{H^{2} \Xi_{a}^{2} \Xi_{b}^{2} a^{2} b^{2}\left(a^{2}-b^{2}\right)^{2} B_{1} B_{2}} d t^{2}+B_{2}\left(d \phi_{2}+v_{21} d \phi_{1}+v_{20} d t\right)^{2} \\
& \left.+B_{1}\left(d \phi_{1}+v_{10} d t\right)^{2}\right]
\end{aligned}
$$

where $B_{1}, B_{2}, v_{10}, v_{20}$ and $v_{21}$ can be determined by comparing (5.5) with (5.1). Because the explicit expressions of these coefficients are lengthy and not very illuminating, we do not list them here. Moreover, we notice a sign error in [55] for the term $\sim d t^{2}$ in (5.5).

The thermodynamic quantities can be expressed as

$$
\begin{aligned}
E & =\frac{\pi}{3 \Xi_{a} \Xi_{b}}\left[2 m\left(\frac{1}{\Xi_{a}}+\frac{1}{\Xi_{b}}\right)+q\left(1+\frac{\Xi_{a}}{\Xi_{b}}+\frac{\Xi_{b}}{\Xi_{a}}\right)\right], \\
S & =\frac{2 \pi^{2}\left[\left(r_{+}^{2}+a^{2}\right)\left(r_{+}^{2}+b^{2}\right)+q r_{+}\right]}{3 \Xi_{a} \Xi_{b}}, \\
T & =\frac{2 r_{+}^{2}\left(1+g^{2} r_{+}^{2}\right)\left(2 r_{+}^{2}+a^{2}+b^{2}\right)-\left(1-g^{2} r_{+}^{2}\right)\left(r_{+}^{2}+a^{2}\right)\left(r_{+}^{2}+b^{2}\right)+4 q g^{2} r_{+}^{3}-q^{2} g^{2}}{4 \pi r_{+}\left[\left(r_{+}^{2}+a^{2}\right)\left(r_{+}^{2}+b^{2}\right)+q r_{+}\right]} \\
J_{1} & =\frac{2 \pi m a\left(1+\Xi_{b} \sinh ^{2}(\delta)\right)}{3 \Xi_{a}^{2} \Xi_{b}}, \quad J_{2}=\frac{2 \pi m b\left(1+\Xi_{a} \sinh ^{2}(\delta)\right)}{3 \Xi_{b}^{2} \Xi_{a}}, \\
\Omega_{1} & =\frac{a\left[\left(1+g^{2} r_{+}^{2}\right)\left(r_{+}^{2}+b^{2}\right)+q g^{2} r_{+}\right]}{\left(r_{+}^{2}+a^{2}\right)\left(r_{+}^{2}+b^{2}\right)+q r_{+}}, \quad \Omega_{2}=\frac{b\left[\left(1+g^{2} r_{+}^{2}\right)\left(r_{+}^{2}+a^{2}\right)+q g^{2} r_{+}\right]}{\left(r_{+}^{2}+a^{2}\right)\left(r_{+}^{2}+b^{2}\right)+q r_{+}} \\
Q & =\frac{2 \pi m \sinh (\delta) \cosh (\delta)}{\Xi_{a} \Xi_{b}}, \quad \Phi=\frac{2 m r_{+} \sinh (\delta) \cosh (\delta)}{\left(r_{+}^{2}+a^{2}\right)\left(r_{+}^{2}+b^{2}\right)+q r_{+}} .
\end{aligned}
$$

This class of asymptotically $\mathrm{AdS}_{6}$ black hole solutions is characterized by four parameters $(m, \delta, a, b)$. The BPS limit can be obtained by imposing the following condition

$$
e^{2 \delta}=1+\frac{2}{(a+b) g} .
$$


The absence of the naked closed timelike curves (CTCs) for these supersymmetric black holes requires an additional condition

$$
q=\frac{\Xi_{a+} \Xi_{b+}(a+b) r_{+}}{(1+a g+b g) g}
$$

where in the BPS limit

$$
r_{+} \equiv \sqrt{\frac{a b}{1+a g+b g}}, \quad \Xi_{a+} \equiv 1+a g, \quad \Xi_{b+} \equiv 1+b g .
$$

\subsection{Gravitational Cardy limit}

The Cardy-like limit for the 5d SCFT was defined in [6]

$$
\left|\omega_{i}\right| \ll 1, \quad \Delta \sim \mathcal{O}(1), \quad(i=1,2) .
$$

Using the following relations [48]

$$
\omega_{i}=-\lim _{T \rightarrow 0} \frac{\Omega_{i}-\Omega_{i}^{*}}{T}, \quad \Delta=-\lim _{T \rightarrow 0} \frac{\Phi-\Phi^{*}}{T},
$$

with $\Omega_{i}^{*}=g$ and $\Phi^{*}=1$ denoting the BPS values of $\Omega_{i}$ and $\Phi$, we can find the gravitational counterpart of the Cardy-like limit (5.10)

$$
\left|\left(\frac{\partial \Omega_{i}}{\partial T}\right)_{T=0}\right| \ll 1,\left.\quad \frac{\partial \Phi}{\partial T}\right|_{T=0} \sim \mathcal{O}(1) .
$$

The equations $\left(\frac{\partial \Omega_{i}}{\partial T}\right)_{*}=0$ have the roots

$$
a=\frac{1}{g} \quad \text { and } \quad b=\frac{1}{g} .
$$

Hence, the gravitational Cardy limit for the class of asymptotically $\mathrm{AdS}_{6}$ black holes (5.1) is

$$
a \rightarrow \frac{1}{g} \text { and } b \rightarrow \frac{1}{g} .
$$

Similar to the black hole solutions in the previous sections, we have

$$
a_{i} g \rightarrow 1,
$$

where $a_{i}=\{a, b\}$. We can introduce small parameters to denote the deviations from this limit, i.e.,

$$
a=\frac{1}{g}+\epsilon, \quad b=\frac{1}{g}+\epsilon+\eta, \quad \text { with } 0 \neq \eta \ll \epsilon .
$$

Expanding in $\epsilon$ after expanding in $\eta$, we find the thermodynamic quantities (5.6) in the BPS and gravitational Cardy limit (5.16) to the leading order

$$
\begin{aligned}
S_{*} & =\frac{4 \pi^{2}}{9 g^{6} \epsilon^{2}}+\mathcal{O}\left(\epsilon^{-1}\right), \\
J_{1}^{*} & =-\frac{8 \pi}{9 \sqrt{3} g^{7} \epsilon^{3}}+\mathcal{O}\left(\epsilon^{-2}\right), \\
J_{2}^{*} & =-\frac{8 \pi}{9 \sqrt{3} g^{7} \epsilon^{3}}+\mathcal{O}\left(\epsilon^{-2}\right), \\
Q^{*} & =\frac{2 \pi}{\sqrt{3} g^{5} \epsilon^{2}}+\mathcal{O}\left(\epsilon^{-1}\right),
\end{aligned}
$$


which are consistent with $[6,41]$ and the Cardy-like limit on the field theory side (5.10)

$$
\omega_{i}^{*} \sim \epsilon, \quad \Delta_{*} \sim \mathcal{O}(1)
$$

\subsection{Black hole solution in the near-horizon + gravitational Cardy limit}

In the previous subsection, we have obtained the gravitational Cardy limit for the parameters on the gravity side. In this subsection, we discuss how the near-horizon metric changes when taking the gravitational Cardy limit. In appendix A.4, we verify explicitly that the resulting background is a solution of the $6 \mathrm{~d}$ gauged supergravity equations of motion. In the following, we implement the gravitational Cardy limit in the space of parameters, which further simplifies the geometry.

We apply the following scaling near the horizon $r_{+}(5.9)$ to the asymptotically $\mathrm{AdS}_{6}$ black hole metric (5.5) in the BPS limit

$$
r \rightarrow r_{+}+\lambda \widetilde{r}, \quad t \rightarrow \frac{\tilde{t}}{\lambda}, \quad \phi_{1} \rightarrow \widetilde{\phi}_{1}+g \frac{\tilde{t}}{\lambda}, \quad \phi_{2} \rightarrow \widetilde{\phi}_{2}+g \frac{\tilde{t}}{\lambda}
$$

with $\lambda \rightarrow 0$, and then take the $\mathrm{AdS}_{6}$ gravitational Cardy limit (5.16). To the leading order in $\epsilon$ and $\eta$, the metric becomes

$$
\begin{aligned}
d s^{2}= & -\frac{\sqrt{3} g^{2}}{4} \sqrt{\left(1+3 g^{2} y^{2}\right)\left(1+3 g^{2} z^{2}\right)\left[3+3 g^{4} y^{2} z^{2}+g^{2}\left(y^{2}+z^{2}\right)\right]} \widetilde{r}^{2} d \widetilde{t}^{2} \\
& +\left(1+3 g^{2} y^{2}\right)\left(1+3 g^{2} z^{2}\right) H_{*}(y, z) \frac{d \widetilde{r}^{2}}{144 g^{2} \widetilde{r}^{2}} \\
& +\frac{g^{2}\left(1+3 g^{2} y^{2}\right)\left(z^{2}-y^{2}\right)}{3\left(1-g^{2} y^{2}\right)^{3}} H_{*}(y, z) d y^{2}+\frac{g^{2}\left(1+3 g^{2} z^{2}\right)\left(z^{2}-y^{2}\right)}{3\left(g^{2} z^{2}-1\right)^{3}} H_{*}(y, z) d z^{2} \\
& +\frac{4\left(1-g^{2} y^{2}\right)^{2}\left(1-g^{2} z^{2}\right)^{2}\left[z^{2}+y^{2}\left(1+2 g^{2} z^{2}\right)\right]}{3 g^{4}\left[3+3 g^{4} y^{2} z^{2}+g^{2}\left(y^{2}+z^{2}\right)\right]^{2} \epsilon^{2} \eta^{2}} H_{*}(y, z)\left(d \widetilde{\phi}_{1}-d \widetilde{\phi}_{2}\right)^{2} \\
& +\frac{\left(1-g^{2} y^{2}\right)\left(g^{2} z^{2}-1\right)\left(1+3 g^{2} y^{2}\right)\left(1+3 g^{2} z^{2}\right)}{12 g^{6}\left(y^{2}+z^{2}+2 g^{2} y^{2} z^{2}\right) \epsilon^{2}} H_{*}(y, z)\left(d \widetilde{\phi}_{1}-\frac{\sqrt{3}}{2} g^{3} \epsilon \widetilde{r} d \widetilde{t}\right)^{2},
\end{aligned}
$$

where

$$
H_{*}(y, z) \equiv \sqrt{1+\frac{8}{\left(1+3 g^{2} y^{2}\right)\left(1+3 g^{2} z^{2}\right)}} .
$$

Defining

$$
\tau \equiv 6 g^{2} \widetilde{t}, \quad \chi \equiv \frac{\widetilde{\phi}_{1}-\widetilde{\phi}_{2}}{g \eta}
$$


we can rewrite the metric (5.20) as

$$
\begin{aligned}
d s^{2}= & H_{*}(y, z)\left[\frac{\left(1+3 g^{2} y^{2}\right)\left(1+3 g^{2} z^{2}\right)}{144 g^{2}}\left(-\widetilde{r}^{2} d \tau^{2}+\frac{d \widetilde{r}^{2}}{\widetilde{r}^{2}}\right)\right. \\
& +\frac{g^{2}\left(1+3 g^{2} y^{2}\right)\left(z^{2}-y^{2}\right)}{3\left(1-g^{2} y^{2}\right)^{3}} d y^{2}+\frac{g^{2}\left(1+3 g^{2} z^{2}\right)\left(z^{2}-y^{2}\right)}{3\left(g^{2} z^{2}-1\right)^{3}} d z^{2} \\
& +\frac{4\left(1-g^{2} y^{2}\right)^{2}\left(1-g^{2} z^{2}\right)^{2}\left[z^{2}+y^{2}\left(1+2 g^{2} z^{2}\right)\right]}{3 g^{2}\left[3+3 g^{4} y^{2} z^{2}+g^{2}\left(y^{2}+z^{2}\right)\right]^{2} \epsilon^{2}} d \chi^{2} \\
& \left.+\frac{\left(1-g^{2} y^{2}\right)\left(g^{2} z^{2}-1\right)\left(1+3 g^{2} y^{2}\right)\left(1+3 g^{2} z^{2}\right)}{12 g^{6}\left(y^{2}+z^{2}+2 g^{2} y^{2} z^{2}\right) \epsilon^{2}}\left(d \widetilde{\phi}_{1}-\frac{\sqrt{3}}{12} g \epsilon \widetilde{r} d \tau\right)^{2}\right] .
\end{aligned}
$$

\subsection{Black hole entropy from Cardy formula}

For the asymptotically $\mathrm{AdS}_{6}$ black holes discussed in this section, we apply the Cardy formula to the near-horizon metric only after taking the gravitational Cardy limit. More explicitly, we first rewrite the metric (5.23) from Poincaré coordinates $(\widetilde{r}, \tau)$ to global coordinates $(\hat{r}, \hat{t})$ using the relations $(2.44)-(2.46)$. Consequently, the near-horizon metric in the gravitational Cardy limit (5.23) becomes

$$
\begin{aligned}
d s^{2}= & H_{*}(y, z)\left[\frac{\left(1+3 g^{2} y^{2}\right)\left(1+3 g^{2} z^{2}\right)}{144 g^{2}}\left(-\left(1+\hat{r}^{2}\right) d \hat{t}^{2}+\frac{d \hat{r}^{2}}{1+\hat{r}^{2}}\right)\right. \\
& +\frac{g^{2}\left(1+3 g^{2} y^{2}\right)\left(z^{2}-y^{2}\right)}{3\left(1-g^{2} y^{2}\right)^{3}} d y^{2}+\frac{g^{2}\left(z^{2}-y^{2}\right)\left(1+3 g^{2} z^{2}\right)}{3\left(g^{2} z^{2}-1\right)^{3}} d z^{2} \\
& +\frac{4\left(1-g^{2} y^{2}\right)^{2}\left(1-g^{2} z^{2}\right)^{2}\left[z^{2}+y^{2}\left(1+2 g^{2} z^{2}\right)\right]}{3 g^{2}\left[3+3 g^{4} y^{2} z^{2}+g^{2}\left(y^{2}+z^{2}\right)\right]^{2} \epsilon^{2}} d \chi^{2} \\
& \left.+\frac{\left(1-g^{2} y^{2}\right)\left(g^{2} z^{2}-1\right)\left(1+3 g^{2} y^{2}\right)\left(1+3 g^{2} z^{2}\right)}{12 g^{6}\left(y^{2}+z^{2}+2 g^{2} y^{2} z^{2}\right) \epsilon^{2}}\left(d \hat{\psi}-\frac{\sqrt{3}}{12} g \epsilon \hat{r} d \hat{t}\right)^{2}\right],
\end{aligned}
$$

where

$$
\hat{\psi} \equiv \widetilde{\phi}_{1}-\frac{\sqrt{3}}{12} g \epsilon \gamma .
$$

Applying the same formalism in subsection 2.4 and choosing appropriate ranges of $y$ and $z$, we obtain the central charge and the extremal Frolov-Thorne temperature in the near-horizon region of the asymptotically $\mathrm{AdS}_{6}$ BPS black holes as follows:

$$
c_{L}=\frac{5 \pi}{3 \sqrt{3} g^{5} \epsilon}, \quad T_{L}=\frac{4 \sqrt{3}}{5 \pi g \epsilon} .
$$

Using the Cardy formula, we can compute the black hole entropy of the asymptotically $\mathrm{AdS}_{6}$ BPS black holes:

$$
S_{B H}=\frac{\pi^{2}}{3} c_{L} T_{L}=\frac{4 \pi^{2}}{9 g^{6} \epsilon^{2}},
$$

which is the same as the black hole entropy in the gravitational Cardy limit (5.17) from the gravity side. 


\subsection{Comparison with results from boundary CFT}

For the asymptotically $\mathrm{AdS}_{6}$ BPS black holes, it was shown in $[6,14,48]$ that the entropies of these black holes can be obtained from the boundary $5 \mathrm{~d} \mathcal{N}=1$ superconformal field theories by extremizing an entropy function, which has also been studied in [44]. We can first compute the free energy in the large- $N$ limit using the $5 \mathrm{~d}$ superconformal index. The entropy function is then defined as a Legendre transform of the free energy in the large- $N$ limit

$$
S\left(\Delta_{I}, \omega_{i}\right)=-\frac{i \pi}{81 g^{4} G} \frac{\Delta^{3}}{\omega_{1} \omega_{2}}+Q \Delta+\sum_{i=1}^{2} J_{i} \omega_{i}+\Lambda\left(\Delta-\sum_{i=1}^{2} \omega_{i}-2 \pi i\right) .
$$

In the Cardy-like limit (5.10)

$$
\omega \sim \epsilon, \quad \Delta_{I} \sim \mathcal{O}(1),
$$

we can read off from the entropy function (5.28)

$$
S \sim \frac{1}{\epsilon^{2}}, \quad J \sim \frac{1}{\epsilon^{3}}, \quad Q_{I} \sim \frac{1}{\epsilon^{2}},
$$

which have been summarized in table 1 .

Similar to $\mathrm{AdS}_{4,5,7}$, for $\mathrm{AdS}_{6}$ the electric charge $Q$ and the angular momenta $J_{i}$ are real, while the chemical potential $\Delta$ and the angular velocities $\omega_{i}$ can be complex, and so can the entropy function $S$. By requiring that the black hole entropy $S_{B H}$ to be real after extremizing the entropy function $S$, we obtain one more constraint on $Q$ and $J_{i}$. More precisely, the asymptotically $\mathrm{AdS}_{6}$ black hole entropy and the corresponding constraint are given implicitly by the following two relations $[6,48]$

$$
\begin{aligned}
S_{B H}^{3}-\frac{2 \pi^{2}}{3 g^{4} G_{N}} S_{B H}^{2}-12 \pi^{2}\left(\frac{Q}{3 g}\right)^{2} S_{B H}+\frac{8 \pi^{4}}{3 g^{4} G_{N}} J_{1} J_{2} & =0, \\
\frac{Q}{3 g} S_{B H}^{2}+\frac{2 \pi^{2}}{9 g^{4} G_{N}}\left(J_{1}+J_{2}\right) S_{B H}-\frac{4 \pi^{2}}{3}\left(\frac{Q}{3 g}\right)^{3} & =0,
\end{aligned}
$$

which are consistent with both the thermodynamic quantities on the gravity side (5.6) and (5.17) as well as the black hole entropy in the gravitational Cardy limit from the Cardy formula (5.27) under the $\mathrm{AdS}_{6} / \mathrm{CFT}_{5}$ dictionary of parameters [6, 48]

$$
\frac{1}{g^{4} G_{N}} \sim N^{5 / 2}
$$

\section{Discussion}

In this paper, we have discussed the near-horizon gravitational Cardy limit of asymptotically $\mathrm{AdS}_{4,5,6,7}$ black holes. The gravitational Cardy limit can be written universally as $\left|a_{i} g\right| \rightarrow 1$, where $a_{i}$ are parametrize angular momenta in units of the inverse AdS radius, $g$, for all the black hole solutions we analyzed. As we have explicitly shown in these examples, the gravitational Cardy limit leads to an $\mathrm{AdS}_{3}$ geometry near the horizon and is effectively an additional limit on the independent parameters of the black hole solutions. The macroscopic Bekenstein-Hawking entropy of asymptotically AdS black holes has recently been 
given a microscopic foundation using the dual boundary $\mathrm{CFT}_{3,4,5,6}$. Our work relies on a near-horizon $\mathrm{AdS}_{3}$ geometry and we provide an effective microscopic description via the $\mathrm{CFT}_{2}$ Cardy formula obtained from the algebra of asymptotic symmetries.

It is instructive to point out various analogies with the previous instance when string theory answered explicitly the problem of microstate counting for black hole entropy. In the mid 90's, Strominger and Vafa [23] used the full machinery of D-brane technology to provide a microscopic description of the Bekenstein-Hawking entropy of a class of asymptotically flat black holes. Viewing the D-brane description as the UV complete description of gravity, the analogy with the current developments is that the microscopic description of the entropy of $\mathrm{AdS}_{d+1}$ black holes in terms of field theory degrees of freedom in the dual $\mathrm{CFT}_{d}$ boundary theory is the UV complete description. After the UV complete description of the 90's, Strominger went on to provide a universal description [22], based only on the near-horizon symmetries exploiting the $\mathrm{AdS}_{3}$ near-horizon region and the asymptotic symmetry algebra computation of Brown and Henneaux [56]. Similar symmetry-based approaches were shown to apply to a wide variety of black holes by Carlip [57]. The results presented in [19, 20] and in this manuscript show that we can understand the entropy of asymptotically AdS black holes based only on near-horizon symmetries via the Kerr/CFT correspondence.

The satisfying aspect of this point of view resides in the separation-of-scales principle. Such a universal feature of gravity as the Bekenstein-Hawking entropy formula can certainly be explained using UV complete formulations of quantum gravity but must also be understood without recourse to the existence of such a UV complete theory and could be determined strictly from low energy symmetry principles.

The point of view advocated in this manuscript leads to a number of interesting questions some of which we now describe. It would be interesting to understand the field theory counterpart of the locally $\mathrm{AdS}_{3}$ near-horizon region that arises from the Bardeen-Horowitz limit plus the gravitational Cardy limit. It clearly suggests the existence of an effective $\mathrm{CFT}_{2}$ which we have used to microscopically compute the entropy but whose further details we do not know. Some aspects of this effective $\mathrm{CFT}_{2}$ were studied in $[58,59]$ for the $\mathrm{AdS}_{5}$ and the $\mathrm{AdS}_{4}$ black holes, but it required going away from extremality. In the bigger picture described above, understanding how this effective $\mathrm{CFT}_{2}$ embeds in the boundary $\mathrm{CFT}_{d}$ is the dual to finding the UV complete description of the gravitational theory living near the horizon - a worthy challenge. Along these lines, in this manuscript, we have only discussed the asymptotically AdS black holes in the BPS limit, hence at zero temperature. It would be interesting to extend the discussion to near-extremal asymptotically $\operatorname{AdS}_{4,6,7}$ black holes and to reproduce the Bekenstein-Hawking entropy formula from a near-horizon Cardy formula. When higher-derivative terms are included in the gravity theory, the black hole entropy does not obey the area law. It was shown in [60] that the central charge of the near-horizon asymptotic Virasoro symmetry also gets modified in the gravity with higher-derivative terms, while the Frolov-Thorne temperature and the Cardy formula still hold. Other higher-derivative aspects of $\mathrm{AdS}_{4}$ black holes were recently considered [61, 62]. A tantalizing property of higher-derivative corrections in $\mathrm{AdS}_{5}$ black holes was recently reported in [63], which showed that the leading $\alpha^{\prime}$-correction is absent in the BPS limit. This suggests that the central charge of the near-horizon asymptotic Virasoro symmetry remains the same in this case. 
There is another line of attack that is worth sketching. Recall that the original setup for Cardy-like limits is 2d CFT. In this case, one simply has a formula for $\mathrm{CFT}_{2}$ on $S^{1} \times S^{1}$ which effectively relates the high energy and low energy degrees of freedom. It is fair to think of this relation as a UV/IR relation with the important characteristic of being controlled by the anomaly, $c$. Similar formulas have been developed in higher dimensions by Di Pietro and Komargodski in [64] and further clarified in [31, 65, 66]. In particular, in four dimensions they found an effective description of theories in $S^{1} \times M^{3}$ whose effective action is controlled by anomaly coefficients. A similar analysis has been rigorously performed for a set of six-dimensional theories $[4,8,67]$. More closely related to the questions we addressed in this paper is the recent work of Seok Kim and collaborators who have used an effective low energy action approach to find the leading term in the entropy function for the Cardy-like limit, first in $\mathcal{N}=4 \mathrm{SYM}$ as well as in the $6 \mathrm{~d} \mathcal{N}=(2,0)$ SCFT living on $N$ M5-branes [4], and later for a more generic $4 \mathrm{~d} \mathcal{N}=1$ situation [33]. These developments point to the possibility that the Cardy-like limit may be understood as the leading term in an effective field theory expansion. Although for these cases in the BPS limit the Cardy-like free energy has been derived from the effective quantum field theory approach, higher order corrections as well as finite temperatures should be taken into account to go beyond the leading order in the BPS limit. It would be quite interesting to explore such possibilities on the field theory side and, ultimately, connect it with a more standard hydrodynamics approach on the gravity side [68, 69].

Finally, it would be nice to develop what seems like a more natural $\mathrm{AdS}_{2}$ or SYK approach to the entropy of extremal AdS black holes as described in figure 1. Some interesting work along this direction was performed in [70] for $\mathrm{AdS}_{5}$ and more recently in [71] for $\mathrm{AdS}_{4}$. Finding the connection between the $\mathrm{AdS}_{2}$ and $\mathrm{AdS}_{3}$ low energy descriptions in more details is an interesting problem.

\section{Acknowledgments}

We would like to thank Arash Arabi Ardehali, Sunjin Choi, Zhihao Duan, Martin Fluder, Alfredo González Lezcano, Simeon Hellerman, Kentaro Hori, Seyed Morteza Hosseini, Cindy Keeler, Joonho Kim, Seok Kim, Sung-Soo Kim, Finn Larsen, Kimyeong Lee, Sungjay Lee, Yang Lei, June Nahmgoong, Ioannis Papadimitriou, Wei Song, Futoshi Yagi, Junya Yagi, Masahito Yamazaki, Piljin Yi and Yang Zhou for many helpful discussions. We also would like to thank David Chow for very useful communications. This work was supported in part by the U.S. Department of Energy under grant DE-SC0007859. M.D. was supported by the NSF Graduate Research Fellowship Program under NSF Grant Number: DGE 1256260. J.N. was also supported by a Van Loo Postdoctoral Fellowship and he would like to thank Rutgers University, Arizona State University, UESTC, Southwest Jiaotong University, Sichuan University, KIAS and Kavli IPMU for warm hospitality during various stages of this work. 


\section{A Verifying the equations of motion for the near-horizon}

Gravitational theories are nonlinear and, therefore, a truncated sector of a solution need not be a solution itself. For that reason, we explicitly verify that, in each instance, the near-horizon limit satisfies the equations of motion. This fact alone should inspire trust in the consistency of the resulting geometry and the potential existence and closure of a dual field theory sector. Returning to the analogy with the BMN paradigm, this is equivalent to checking the equations of motion for the plane wave background [17].

\section{A.1 $A d S_{5}$}

We verify the equations of motion for the near-horizon geometry for $A d S_{5}$. The Lagrangian describing the solution in [29] is

$$
\mathcal{L}=\left(R+12 g^{2}\right) * 1-\frac{1}{2} * F \wedge F+\frac{1}{3 \sqrt{3}} F \wedge F \wedge A,
$$

and the equations of motion are

$$
R_{a b}-\frac{1}{2} F_{a c} F_{b}{ }^{c}+\frac{1}{3} g_{a b}\left(\frac{1}{4} F^{2}+12 g^{2}\right)=0, \quad d \star F-\frac{1}{\sqrt{3}} F \wedge F=0 .
$$

In order to facilitate the computation, we turn to a vielbein description for the near-horizon geometry,

$$
\begin{aligned}
& e_{1}=\sqrt{\frac{a}{10 a g^{2}+2 g}} \tilde{r} d \tau, \\
& e_{2}=\sqrt{\frac{a}{10 a g^{2}+2 g}} \frac{d \tilde{r}}{\tilde{r}}, \\
& e_{3}=\sqrt{\frac{2 a}{g-a g^{2}} d \theta} \\
& e_{4}=p_{1}\left(p_{2}\left((-3 a g \cos 2 \theta+a g-4) d \tilde{\psi}-6 a g \sin ^{2} \theta d \tilde{\phi}\right)+3 a\left(a^{2} g^{2}+a g-2\right) \tilde{r} d \tau\right), \\
& e_{5}=p_{3}\left(3 a(1-a g) \tilde{r} d \tau+2 p_{2} d \tilde{\phi}\right),
\end{aligned}
$$

where

$$
\begin{aligned}
& p_{1}=-\frac{\cos \theta}{(1-a g)(5 a g+1) \sqrt{2(a g+2)(3 a g \cos (2 \theta)-a g+4)}}, \\
& p_{2}=\sqrt{\frac{a(a g+2)}{g}(5 a g+1),} \\
& p_{3}=\frac{\sin \theta}{(5 a g+1) \sqrt{(1-a g)(3 a g \cos (2 \theta)-a g+4)}} .
\end{aligned}
$$

Note that this coframe describes the near-horizon, which is computed using [21]. After applying the near-horizon geometry and gauge fixing, the gauge potential is

$$
\begin{aligned}
A_{(1), \text { near }}= & -\frac{\sqrt{6}(1-a g)}{\sqrt{a g+2} \sqrt{5 a g+1}} e_{1}-\frac{\sqrt{6 a g} \cos \theta}{\sqrt{3 a g \cos 2 \theta-a g+4}} e_{4} \\
& -\frac{2 \sqrt{3 a g(1-a g)} \sin \theta}{(\sqrt{a g+2} \sqrt{3 a g \cos 2 \theta-a g+4}} e_{5} .
\end{aligned}
$$


Note that the exterior derivative and the near-horizon geometry limit commute to give an equivalent expression for the gauge field,

$$
\begin{aligned}
F_{(2), \text { near }}=d A_{(1), \text { near }}= & \frac{\sqrt{3 a g(a g+2)}}{a} e_{1} \wedge e_{2}+2 g \sin \theta \sqrt{\frac{3(1-a g)}{3 a g \cos 2 \theta-a g+4}} e_{3} \wedge e_{4} \\
& +g \cos \theta \sqrt{\frac{6(a g+2)}{(3 a g \cos 2 \theta-a g+4)}} e_{3} \wedge e_{5} .
\end{aligned}
$$

Then,

$$
\begin{aligned}
F_{(2), \text { near }} \wedge F_{(2), \text { near }}=6 g^{3 / 2} \frac{\sqrt{a g+2}}{\sqrt{a(3 a g \cos 2 \theta-a g+4)}} & \left(2 \sqrt{1-a g} \sin \theta e_{1} \wedge e_{2} \wedge e_{3} \wedge e_{4}\right. \\
& \left.-\sqrt{2 a g+4} \cos \theta e_{1} \wedge e_{2} \wedge e_{3} \wedge e_{5}\right) .
\end{aligned}
$$

The other term gives

$$
\begin{aligned}
d \star F_{(2), \text { near }}= & d\left[\sqrt{6} g \cos \theta \sqrt{\frac{a g+2}{3 a g \cos 2 \theta-a g+4}} e_{1} \wedge e_{2} \wedge e_{4}-\frac{\sqrt{3 a g(a g+2)}}{a} e_{3} \wedge e_{4} \wedge e_{5}\right. \\
& \left.+2 \sqrt{3} g \sin \theta \sqrt{\frac{1-a g}{3 a g \cos 2 \theta-a g+4}} e_{1} \wedge e_{2} \wedge e_{5}\right] \\
= & \frac{2 \sqrt{3(a g+2)} g^{3 / 2}}{\sqrt{a(3 a g \cos 2 \theta-a g+4)}}\left(2 \sqrt{1-a g} \sin \theta e_{1} \wedge e_{2} \wedge e_{3} \wedge e_{4}\right. \\
& \left.-\sqrt{2 a g+4} \cos \theta e_{1} \wedge e_{2} \wedge e_{3} \wedge e_{5}\right) .
\end{aligned}
$$

Comparing equations (A.7) and (A.8), we can see that the equation of motion for the gauge potential is satisfied. For the Einstein equations, the geometric data we need are the nonzero components of the Ricci tensor

$$
\begin{aligned}
& R_{00, \text { near }}=-R_{11, \text { near }}=\frac{g(11 a g+4)}{2 a}, \\
& R_{22, \text { near }}=-\frac{g(5 a g-2)}{2 a}, \\
& R_{33, \text { near }}=-\frac{g\left(9 a^{2} g^{2} \cos 2 \theta-a^{2} g^{2}+14 a g-4\right)}{a(3 a g \cos 2 \theta-a g+4)}, \\
& R_{34, \text { near }}=-\frac{3 \sqrt{2} g^{2} \sqrt{2-a g-a^{2} g^{2}} \sin \theta \cos \theta}{3 a g \cos 2 \theta-a g+4}, \\
& R_{44, \text { near }}=-\frac{g\left(21 a^{2} g^{2} \cos 2 \theta-11 a^{2} g^{2}-12 a g \cos 2 \theta+28 a g-8\right)}{2 a(3 a g \cos 2 \theta-a g+4)} .
\end{aligned}
$$


We also need the explicit nonzero contractions of the gauge field $F_{a c, \text { near }} F_{b}{ }^{c}$,near $\equiv \mathcal{F}_{a b \text {,near }}$

$$
\begin{aligned}
& \mathcal{F}_{00, \text { near }}=\frac{3 g(a g+2)}{a}, \\
& \mathcal{F}_{11 \text {,near }}=-\mathcal{F}_{00, \text { near }}, \quad \mathcal{F}_{22 \text {,near }}=3 g^{2}, \\
& \mathcal{F}_{33, \text { near }}=\frac{12 g^{2}(1-a g) \sin ^{2} \theta}{3 a g \cos 2 \theta-a g+4}, \\
& \mathcal{F}_{34, \text { near }}=-\frac{3 g^{2} \sin 2 \theta \sqrt{2(1-a g)(2+a g)}}{3 a g \cos 2 \theta-a g+4}, \\
& \mathcal{F}_{44, \text { near }}=\frac{6 g^{2}(a g+2) \cos ^{2} \theta}{3 a g \cos 2 \theta-a g+4}, \quad F_{a b, \text { near }} F^{a b, \text { near }}=-\frac{12 g}{a} .
\end{aligned}
$$

The equations of motion are then verified once we impose these expressions.

\section{A.2 $A d S_{4}$}

The $4 \mathrm{~d} \mathcal{N}=4$ gauged supergravity can be obtained by the truncation of the $11 \mathrm{~d}$ supergravity [45]

$$
\begin{aligned}
\mathcal{L}_{4}= & R * 1-\frac{1}{2} * \mathrm{~d} \varphi \wedge \mathrm{d} \varphi-\frac{1}{2} e^{2 \varphi} * \mathrm{~d} \chi \wedge \mathrm{d} \chi-\frac{1}{2} e^{-\varphi} * F_{(2) 2} \wedge F_{(2) 2}-\frac{1}{2} \chi F_{(2) 2} \wedge F_{(2) 2} \\
& -\frac{1}{2\left(1+\chi^{2} e^{2 \varphi}\right)}\left(e^{\varphi} * F_{(2) 1} \wedge F_{(2) 1}-e^{2 \varphi} \chi F_{(2) 1} \wedge F_{(2) 1}\right) \\
& -g^{2}\left(4+2 \cosh \varphi+e^{\varphi} \chi^{2}\right) * 1
\end{aligned}
$$

where $\varphi$ and $\chi$ are the dilaton and axion. The subscript in parenthesis denotes the degree of the form. The solution has two pairwise equal charges and therefore two gauge potential $A_{(1) 1}$ and $A_{(1) 2}$. The equations of motion are

$$
\begin{aligned}
0= & \mathrm{d}\left(\frac{1}{1+\chi^{2} e^{2 \varphi}}\left(e^{\varphi} \star F_{(2) 1}-e^{2 \varphi} \chi F_{(2) 1}\right)\right), \\
0= & \mathrm{d}\left(e^{-\varphi} \star F_{(2) 2}+\chi F_{(2) 2}\right), \\
0= & -\mathrm{d} \star \mathrm{d} \varphi-e^{2 \varphi} \star \mathrm{d} \chi \wedge \mathrm{d} \chi+\frac{1}{2} e^{-\varphi} \star \mathrm{d} A_{(1) 2} \wedge \mathrm{d} A_{(1) 2}-g^{2}\left(2 \sinh \varphi+e^{\varphi} \chi^{2}\right) \star 1 \\
& +\frac{e^{\varphi}\left(e^{2 \varphi} \chi^{2}-1\right)}{2\left(e^{2 \varphi} \chi^{2}+1\right)^{2}} \star \mathrm{d} A_{(1) 1} \wedge \mathrm{d} A_{(1) 1}+\frac{e^{2 \varphi} \chi}{\left(e^{2 \varphi} \chi^{2}+1\right)^{2}} \mathrm{~d} A_{(1) 1} \wedge \mathrm{d} A_{(1) 1}, \\
0= & -\mathrm{d}\left(e^{2 \varphi} \star d \chi\right)-\frac{1}{2} \mathrm{~d} A_{(1) 2} \wedge \mathrm{d} A_{(1) 2}+\frac{e^{3 \varphi} \chi}{\left(e^{2 \varphi} \chi^{2}+1\right)^{2}} \star \mathrm{d} A_{(1) 1} \wedge \mathrm{d} A_{(1) 1}-2 g^{2} e^{\varphi} \chi \star 1 \\
& +\frac{e^{2 \varphi}-e^{4 \varphi} \chi^{2}}{2\left(e^{2 \varphi} \chi^{2}+1\right)^{2}} \mathrm{~d} A_{(1) 1} \wedge \mathrm{d} A_{(1) 1}, \\
0= & R_{a b}-\frac{1}{2} g_{a b} R-\frac{1}{2}\left(\nabla_{a} \varphi \nabla_{b} \varphi-\frac{1}{2} \nabla^{c} \varphi \nabla_{c} \varphi g_{a b}\right)-\frac{1}{2} e^{-\varphi}\left(F_{a c, 2} F_{b, 2}^{c}-\frac{1}{4} F_{c d, 2} F_{2}^{c d} g_{a b}\right) \\
& -\frac{1}{2} e^{2 \varphi}\left(\nabla_{a} \chi \nabla_{b} \chi-\frac{1}{2} \nabla^{c} \chi \nabla_{c} \chi g_{a b}\right)-\frac{e^{\varphi}}{2\left(1+\chi^{2} e^{2 \varphi}\right)}\left(F_{a c, 1} F_{b, 1}^{c}-\frac{1}{4} F_{c d, 1} F_{1}^{c d} g_{a b}\right) \\
& +\frac{1}{2} g^{2}\left(4+2 \cosh \varphi+e^{\varphi} \chi^{2}\right) g_{a b} .
\end{aligned}
$$


The convenient vielbein for this black hole solution is

$$
\begin{aligned}
& e_{1}=G_{1} \sqrt{\cos 2 \theta+x^{2} y^{2}} \tilde{r} d \tau, \\
& e_{2}=G_{1} \sqrt{\cos 2 \theta+x^{2} y^{2}} \frac{d \tilde{r}}{\tilde{r}}, \\
& e_{3}=\sqrt{2} \sqrt{\frac{\cos 2 \theta+x^{2} y^{2}}{h^{2}\left(-2 \cos 2 \theta+x^{4} y^{4}-2 x^{2} y^{2}-1\right)}} d \theta, \\
& e_{4}=G_{4} \sin \theta \sqrt{\frac{-2 \cos 2 \theta+x^{4} y^{4}-2 x^{2} y^{2}-1}{\left(x^{2} y^{2}-1\right)^{2}\left(\cos 2 \theta+x^{2} y^{2}\right)}}\left(G_{5} \tilde{r} d \tau+d \tilde{\phi}\right),
\end{aligned}
$$

where $G_{1}, G_{4}, G_{5}$ are constants

$$
\begin{aligned}
G_{1}= & \frac{x y \sqrt{2\left(x^{4}-1\right)\left(y^{4}-1\right)}}{g}\left(x^{10}\left[y^{10}+y^{6}\right)+x^{8}\left(6 y^{8}-8 y^{4}\right)+x^{6} y^{2}\left(y^{8}-10 y^{4}+5\right)\right. \\
& \left.-2 x^{4}\left(4 y^{8}-7 y^{4}+1\right)+x^{2} y^{2}\left(5 y^{4}-3\right)-2 y^{4}\right]^{-1 / 2}, \\
G_{4}= & \frac{\sqrt{2}\left(x^{2} y^{2}-1\right)}{g\left|x^{2} y^{2}-3\right|}, \\
G_{5}= & G_{1}^{2} \frac{g^{2}\left(x^{2}+y^{2}\right)\left(x^{2} y^{2}-3\right)\left(x^{2} y^{2}-1\right)^{3 / 2}}{\sqrt{2} x y \sqrt{\left(x^{4}-1\right)\left(y^{4}-1\right)}},
\end{aligned}
$$

that depend on the parameters $\delta_{1}, \delta_{2}$ of the solution, which we have redefined as

$$
\delta_{1} \equiv \ln x, \quad \delta_{2} \equiv \ln y .
$$

The scalar fields in the near-horizon are

$$
\begin{aligned}
\chi_{\text {near }} & =-\frac{x \cos (\theta)\left(x^{2}-y^{2}\right)\left(x^{2} y^{2}+1\right) \sqrt{2\left(y^{4}-1\right)\left(x^{2} y^{2}-1\right)}}{y\left(x^{2}\left(y^{4}-1\right) \cos (2 \theta)+x^{6} y^{4}-x^{4} y^{2}-x^{2}+y^{2}\right) \sqrt{x^{4}-1}}, \\
\left(e^{\varphi}\right)_{\text {near }} & =\frac{x^{2}\left(y^{4}-1\right) \cos (2 \theta)+x^{6} y^{4}-x^{4} y^{2}-x^{2}+y^{2}}{x^{2}\left(y^{4}-1\right)\left(\cos (2 \theta)+x^{2} y^{2}\right)},
\end{aligned}
$$

and for the gauge potentials after adding a pure gauge term for convenience,

$$
\begin{aligned}
A_{(1) 1, \text { near }}= & \frac{M_{1}}{\cos (2 \theta)+x^{2} y^{2}}\left[\frac{M_{2}\left(-\left(x^{4}-1\right) y^{2} \cos (2 \theta)+x^{4} y^{2}\left(y^{4}-2\right)-x^{2}\left(y^{4}-1\right)+y^{2}\right)}{G_{1} \sqrt{\cos (2 \theta)+x^{2} y^{2}}} e_{1}\right. \\
& \left.+M_{4} \sin ^{2} \theta\left(\frac{\csc (\theta)\left(x^{2} y^{2}-1\right) \sqrt{\cos (2 \theta)+x^{2} y^{2}}}{G_{4} \sqrt{-2 \cos (2 \theta)+x^{4} y^{4}-2 x^{2} y^{2}-1}} e_{4}-\frac{G_{5}}{G_{1} \sqrt{\cos (2 \theta)+x^{2} y^{2}}} e_{1}\right)\right], \\
A_{(1) 2, \text { near }}= & \frac{M_{1}}{\cos (2 \theta)+x^{2} y^{2}}\left[\frac{M_{2}\left(-x^{2}\left(y^{4}-1\right) \cos (2 \theta)+x^{6} y^{4}-x^{4} y^{2}+x^{2}\left(1-2 y^{4}\right)+y^{2}\right)}{G_{1} \sqrt{\cos (2 \theta)+x^{2} y^{2}}} e_{1}\right. \\
& \left.+M_{4} \sin ^{2} \theta\left(\frac{\csc (\theta)\left(x^{2} y^{2}-1\right) \sqrt{\cos (2 \theta)+x^{2} y^{2}}}{G_{4} \sqrt{-2 \cos (2 \theta)+x^{4} y^{4}-2 x^{2} y^{2}-1}} e_{4}-\frac{G_{5}}{G_{1} \sqrt{\cos (2 \theta)+x^{2} y^{2}}}\right) e_{1}\right],
\end{aligned}
$$


where

$$
\begin{aligned}
& M_{1}=\frac{x^{2} y^{2}-1}{\sqrt{2}}, \\
& M_{2}=-G_{5} \frac{2\left(x^{2} y^{2}+1\right)}{g\left(x^{2}+y^{2}\right)\left(x^{2} y^{2}-3\right)\left(x^{2} y^{2}-1\right)^{2}}, \\
& M_{4}=\frac{4}{3 g-g x^{2} y^{2}} .
\end{aligned}
$$

\section{A.3 $A d S_{7}$}

In this section, we are interested in charged, rotating $A d S_{7}$ black hole solutions as studied in $[51,52]$. The Lagrangian is

$$
\begin{aligned}
\mathcal{L}_{7}= & R \star 1-\frac{1}{2} \sum_{i=1}^{2} \star \mathrm{d} \varphi_{i} \wedge \mathrm{d} \varphi_{i}-\frac{1}{2} \sum_{I=1}^{2} X_{I}^{-2} \star F_{(2)}^{I} \wedge F_{(2)}^{I}-\frac{1}{2} X_{1}^{2} X_{2}^{2} \star F_{(4)} \wedge F_{(4)} \\
& +2 g^{2}\left(8 X_{1} X_{2}+4 X_{1}^{-1} X_{2}^{-2}+4 X_{1}^{-2} X_{2}^{-1}-X_{1}^{-4} X_{2}^{-4}\right) \star 1 \\
& +g F_{(4)} \wedge A_{(3)}+F_{(2)}^{1} \wedge F_{(2)}^{2} \wedge A_{(3)},
\end{aligned}
$$

where

$$
X_{1}=\mathrm{e}^{-\varphi_{1} / \sqrt{10}-\varphi_{2} / \sqrt{2}}, \quad X_{2}=\mathrm{e}^{-\varphi_{1} / \sqrt{10}+\varphi_{2} / \sqrt{2}}, \quad F_{(2)}^{I}=\mathrm{d} A_{(1)}^{I}, \quad F_{(4)}=\mathrm{d} A_{(3)},
$$

where we have fixed a typographical error corresponding to a minus sign in one of the terms in the Lagrangian. The bosonic fields include two scalars $\varphi_{1}$ and $\varphi_{2}$, the graviton, a 3 -form potential $A_{(3)}$, and two $\mathrm{U}(1)$ gauge potentials $A_{(1)}^{I}, I=1,2$. We study two different solutions to this Lagrangian. The first solution is more general with two charges set equal but different angular momenta. The equations of motion corresponding to the scalars and gauge fields are

$$
\begin{aligned}
\square \varphi_{1}= & \frac{8}{\sqrt{10}} g^{2}\left(4 X_{1} X_{2}-3 X_{1}^{-1} X_{2}^{-2}-3 X_{1}^{-2} X_{2}^{-1}+2 X_{1}^{-4} X_{2}^{-4}\right)+\frac{1}{2 \sqrt{10}} \sum_{I=1}^{2} X_{I}^{-2} F^{I a b} F_{a b}^{I} \\
& -\frac{1}{12 \sqrt{10}} X_{1}^{2} X_{2}^{2} F^{a b c d} F_{a b c d}, \\
\square \varphi_{2}= & \frac{1}{2 \sqrt{2}}\left(X_{1}^{-2} F^{1 a b} F_{a b}^{1}-X_{2}^{-2} F^{2 a b} F_{a b}^{2}\right)+4 \sqrt{2} g^{2}\left(X_{1}^{-1} X_{2}^{-2}-X_{1}^{-2} X_{2}^{-1}\right), \\
0= & \mathrm{d}\left(X_{1}^{-2} \star F_{(2)}^{1}\right)-F_{(2)}^{2} \wedge F_{(4)}, \\
0= & \mathrm{d}\left(X_{2}^{-2} \star F_{(2)}^{2}\right)-F_{(2)}^{1} \wedge F_{(4)}, \\
0= & \mathrm{d}\left(X_{1}^{2} X_{2}^{2} \star F_{(4)}\right)-2 g F_{(4)}-F_{(2)}^{1} \wedge F_{(2)}^{2},
\end{aligned}
$$


and for the graviton, we have

$$
\begin{aligned}
0= & R_{a b}-\frac{1}{2} R g_{a b}-g^{2}\left(8 X_{1} X_{2}+4 X_{1}^{-1} X_{2}^{-2}+4 X_{1}^{-2} X_{2}^{-1}-X_{1}^{-4} X_{2}^{-4}\right) g_{a b} \\
& -\sum_{i=1}^{2}\left(\frac{1}{2} \nabla_{a} \varphi_{i} \nabla_{b} \varphi_{i}-\frac{1}{4} \nabla^{c} \varphi_{i} \nabla_{c} \varphi_{i} g_{a b}\right)-\sum_{I=1}^{2} X_{I}^{-2}\left(\frac{1}{2} F_{a}{ }^{I c} F_{b c}^{I}-\frac{1}{8} F^{I c d} F_{c d}^{I} g_{a b}\right) \\
& -X_{1}^{2} X_{2}^{2}\left(\frac{1}{12} F_{a}{ }^{c d e} F_{b c d e}-\frac{1}{96} F^{c d e f} F_{c d e f} g_{a b}\right) .
\end{aligned}
$$

We can truncate this solution as constructed in [51], where the two charges and angular momenta are set equal. This truncation can be done by letting $X=X_{1}=X_{2}=$ $e^{-\varphi / \sqrt{10}}, \varphi_{2}=0$ and $A_{(1)}=A_{(1)}^{1}=A_{(1)}^{2}$ and the Lagrangian of interest becomes

$$
\begin{aligned}
\mathcal{L}_{7}= & R \star 1-\frac{1}{2} \star \mathrm{d} \varphi_{1} \wedge \mathrm{d} \varphi_{1}-X^{-2} \star F_{(2)} \wedge F_{(2)}-\frac{1}{2} X^{4} \star F_{(4)} \wedge F_{(4)} \\
& +2 g^{2}\left(8 X^{2}+8 X^{-3}-X^{-8}\right) \star 1+F_{(2)} \wedge F_{(2)} \wedge A_{(3)}+g F_{(4)} \wedge A_{(3)},
\end{aligned}
$$

and the equations of motion are

$$
\begin{aligned}
& 0=\mathrm{d} \star \mathrm{d} \varphi-\frac{2 X^{-2}}{\sqrt{10}} \star F_{(2)} \wedge F_{(2)}+\frac{2 X^{4}}{\sqrt{10}} \star F_{(4)} \wedge F_{(4)}-\frac{16 g^{2}}{\sqrt{10}}\left(2 X^{2}-3 X^{-3}+X^{-8}\right) \star 1, \\
& 0=d\left(X^{-2} \star F_{(2)}\right)-F_{(2)} \wedge F_{(4)}, \\
& 0=\mathrm{d}\left(X_{1}^{4} \star F_{(4)}\right)-2 g F_{(4)}-F_{(2)}^{1} \wedge F_{(2)}^{2},
\end{aligned}
$$

and for the graviton

$$
\begin{aligned}
0= & R_{a b}-\frac{1}{2} R g_{a b}-\frac{1}{2}\left(\nabla_{a} \varphi \nabla_{b} \varphi-\frac{1}{2} \nabla^{c} \varphi \nabla_{c} \varphi g_{a b}\right)-X^{-2}\left(F_{a}{ }^{c} F_{b c}-\frac{1}{4} F^{c d} F_{c d} g_{a b}\right) \\
& -g^{2}\left(8 X^{2}+8 X^{-3}-X^{-8}\right)-\frac{1}{12} X^{4}\left(F_{a}{ }^{c d e} F_{b c d e}-\frac{1}{8} F^{c d e f} F_{c d e f} g_{a b}\right) .
\end{aligned}
$$

The fields corresponding to the solution in [51] are

$$
\begin{aligned}
X & =H^{-1 / 5} \\
A_{(1)} & =\frac{2 m \sinh (\delta) \cosh (\delta)}{\rho^{4} \Xi H}(d t-a \sigma)+\frac{\alpha_{70} d t}{\Xi_{-}}, \\
A_{(3)} & =\frac{\left(a m \sinh ^{2}(\delta)\right) \sigma \wedge d \sigma}{\rho^{2} \Xi \Xi_{-}}+\alpha_{71} d t \wedge d \theta \wedge d \psi+\alpha_{72} d t \wedge d \xi \wedge d \phi+\alpha_{73} d t \wedge d \xi \wedge d \psi,
\end{aligned}
$$

where we have added pure gauge terms to both potentials $A_{(1)}$ and $A_{(3)}$ for convenience. More precisely, after taking the near-horizon geometry, we have

$$
\alpha_{70}=-1, \quad \alpha_{71}=-\beta \sin \theta \sin ^{2} \xi, \quad \alpha_{72}=\beta \sin 2 \xi, \quad \alpha_{73}=\beta \cos \theta \sin 2 \xi,
$$


where

$$
\beta=-\frac{4\left(e^{2 \delta}-1\right)}{\left(-13 e^{2 \delta}-9 e^{4 \delta}+9 e^{6 \delta}+5\right) g^{2}} .
$$

A convenient vielbein for the near-horizon is

$$
\begin{aligned}
& e_{1}=p_{1} \tilde{r} d \tau \\
& e_{2}=p_{1} \frac{d \tilde{r}}{\tilde{r}} \\
& e_{3}=p_{2}\left(p_{3} \tilde{r} d \tau+p_{4}\left(\sin ^{2} \xi(d \phi+\cos \theta d \psi)+2 d \tilde{\chi}\right)\right), \\
& e_{4}=p_{5} d \xi \\
& e_{5}=p_{5} \sin \xi d \theta \\
& e_{6}=p_{5} \sin \theta \sin \xi d \psi, \\
& e_{7}=p_{5} \sin \xi \cos \xi(d \phi+\cos \theta d \psi),
\end{aligned}
$$

where

$$
\begin{aligned}
& p_{1}=\frac{1}{g} \frac{2^{3 / 5} 3^{1 / 5}\left(e^{2 \delta}+1\right)^{1 / 5}}{\sqrt{6 e^{2 \delta}+27 e^{4 \delta}+43}}, \\
& p_{2}=\frac{1}{2^{2 / 5} 3^{3 / 10} g} \frac{1}{\left(1-3 e^{2 \delta}\right)\left(5-3 e^{2 \delta}\right)} \frac{1}{\sqrt{\left(e^{2 \delta}+1\right)^{3 / 5}\left(9 e^{2 \delta}-7\right)}}, \\
& p_{3}=-\frac{16\left(3 e^{2 \delta}-5\right)^{3 / 2}\left(2 e^{2 \delta}+3 e^{4 \delta}-1\right)}{6 e^{2 \delta}+27 e^{4 \delta}+43} \sqrt{\frac{3\left(9 e^{2 \delta}-7\right)}{\left(-2 e^{2 \delta}+3 e^{4 \delta}-5\right)}}, \\
& p_{4}=-30 e^{2 \delta}+27 e^{4 \delta}+7, \\
& p_{5}=\frac{1}{g} \frac{2^{8 / 5}}{3^{3 / 10}} \frac{\left(e^{2 \delta}+1\right)^{1 / 5}}{\sqrt{\left(-2 e^{2 \delta}+3 e^{4 \delta}-5\right)}} .
\end{aligned}
$$

In the near-horizon limit, the fields in the vielbein basis become

$$
\begin{aligned}
X_{\text {near }}= & \frac{2^{2 / 5}}{3^{1 / 5}\left(e^{2 \delta}+1\right)^{1 / 5}}, \\
A_{(1), \text { near }} & =\frac{2^{2 / 5} 3^{3 / 10}\left(e^{2 \delta}+1\right)^{4 / 5}\left(\left(15-9 e^{2 \delta}\right) e_{1}+\sqrt{6 e^{2 \delta}+27 e^{4 \delta}+43} e_{3}\right)}{\sqrt{44 e^{2 \delta}+210 e^{4 \delta}+108 e^{6 \delta}+243 e^{8 \delta}-301}}, \\
A_{(3), \text { near }} & =\frac{\left(54 e^{2 \delta}+27 e^{4 \delta}-101\right)\left(e_{1} \wedge e_{5} \wedge e_{6}-e_{1} \wedge e_{4} \wedge e_{7}\right)}{2^{9 / 5} 3^{1 / 10}\left(e^{2 \delta}+1\right)^{1 / 10} \sqrt{9 e^{2 \delta}-7} \sqrt{6 e^{2 \delta}+27 e^{4 \delta}+43}} \\
& -\frac{\left(99 e^{2 \delta}-117 e^{4 \delta}+81 e^{6 \delta}-215\right)\left(e_{3} \wedge e_{4} \wedge e_{7}-e_{3} \wedge e_{5} \wedge e_{6}\right)}{2^{4 / 5} 3^{1 / 10}\left(e^{2 \delta}+1\right)^{1 / 10} \sqrt{9 e^{2 \delta}-7}\left(6 e^{2 \delta}+27 e^{4 \delta}+43\right)} .
\end{aligned}
$$

\section{A.4 $A d S_{6}$}

The field content consists of the graviton, a 2 -form $A_{(2)}$, the scalar $\varphi$ and one $\mathrm{U}(1)$ gauge potential $A_{(1)}$ after truncation, as shown in [55]. After appropriate rescaling and gauge 
transformations, the 6d Lagrangian is given by

$$
\begin{aligned}
\mathcal{L}_{6}= & R \star 1-\frac{1}{2} \star \mathrm{d} \varphi \wedge \mathrm{d} \varphi-X^{-2}\left(\star F_{(2)} \wedge F_{(2)}+g^{2} \star A_{(2)} \wedge A_{(2)}\right)-\frac{1}{2} X^{4} \star F_{(3)} \wedge F_{(3)} \\
& +g^{2}\left(9 X^{2}+12 X^{-2}-X^{-6}\right) \star 1-F_{(2)} \wedge F_{(2)} \wedge A_{(2)}-\frac{g^{2}}{3} A_{(2)} \wedge A_{(2)} \wedge A_{(2)},
\end{aligned}
$$

where

$$
X=e^{-\varphi / \sqrt{8}} .
$$

The equations of motion are

$$
\begin{aligned}
G_{a b}= & \frac{1}{2} \nabla_{a} \varphi \nabla_{b} \varphi-\frac{1}{4} \nabla^{c} \varphi \nabla_{c} \varphi g_{a b}+X^{-2}\left(F_{a}^{c} F_{b c}-\frac{1}{4} F^{c d} F_{c d} g_{a b}\right) \\
& +X^{-2} g^{2}\left(A_{a}^{c} A_{b c}-\frac{1}{4} A^{c d} A_{c d} g_{a b}\right)+X^{4}\left(\frac{1}{4} F_{a}^{c d} F_{b c d}-\frac{1}{24} F^{c d e} F_{c d e} g_{a b}\right) \\
& +\frac{g^{2}}{2}\left(9 X^{2}+12 X^{-2}-X^{-6}\right) g_{a b}, \\
\square \varphi= & \frac{1}{\sqrt{8}} X^{-2}\left(F^{a b} F_{a b}+g^{2} A^{a b} A_{a b}\right)-\frac{1}{3 \sqrt{8}} X^{4} F^{a b c} F_{a b c} \\
& +\frac{3}{\sqrt{2}} g^{2}\left(3 X^{2}-4 X^{-2}+X^{-6}\right), \\
\mathrm{d}\left(X^{-2} \star F_{(2)}\right)= & -F_{(2)} \wedge F_{(3)}, \\
\mathrm{d}\left(X^{4} \star F_{(3)}\right)= & -F_{(2)} \wedge F_{(2)}-g^{2} A_{(2)} \wedge A_{(2)}-2 g^{2} X^{-2} \star F_{(2)} .
\end{aligned}
$$

We omit the vielbein and several other details for this black hole as the expressions are quite long. The scalar and the U(1) gauge field in the near-horizon limit take the form

$$
\begin{aligned}
\chi_{\text {near }}^{4} & =\frac{g\left(a\left(b+g y^{2}\right)+y^{2}(b g+1)\right)\left(a\left(b+g z^{2}\right)+z^{2}(b g+1)\right)}{(a g+b g+1)\left(a^{2} b(b g+1)+a\left(b^{2}+b g\left(y^{2}+z^{2}\right)+g^{2} y^{2} z^{2}\right)+g y^{2} z^{2}(b g+1)\right)}, \\
A_{(1), \text { near }} & =W_{1}\left(W_{2} \tilde{r} d \tilde{t}+W_{3} d \tilde{\phi}_{1}+W_{4} d \tilde{\phi}_{2}\right)
\end{aligned}
$$


where

$$
\begin{aligned}
W_{1}= & \frac{\sqrt{a b}}{\sqrt{a g+b g+1}\left(a^{2} b(b g+1)+a\left(b^{2}+b g\left(y^{2}+z^{2}\right)+g^{2} y^{2} z^{2}\right)+g y^{2} z^{2}(b g+1)\right)}, \\
W_{2}= & {\left[( b ^ { 2 } - a ^ { 2 } ) \Xi _ { a } \Xi _ { b } ( a g + b g + 1 ) \left(a^{2}\left(3 b^{2}+b g\left(y^{2}+z^{2}\right)-g^{2} y^{2} z^{2}\right)\right.\right.} \\
& \left.+a\left(b^{2} g\left(y^{2}+z^{2}\right)+b\left(-2 g^{2} y^{2} z^{2}+y^{2}+z^{2}\right)-2 g y^{2} z^{2}\right)-y^{2} z^{2}(b g+1)^{2}\right] \\
& {\left[a^{4} g^{2}\left(b^{2} g^{2}+6 b g+1\right)+2 a^{3} g\left(b^{3} g^{3}+7 b^{2} g^{2}+7 b g+1\right)\right.} \\
& +a^{2}\left(b^{4} g^{4}+14 b^{3} g^{3}+30 b^{2} g^{2}+14 b g+1\right)+2 a b\left(3 b^{3} g^{3}+7 b^{2} g^{2}+7 b g+3\right) \\
& \left.+b^{2}(b g+1)^{2}\right]^{-1}, \\
W_{3}= & \frac{b\left(a^{2}-y^{2}\right)\left(a^{2}-z^{2}\right)\left(b^{2} g^{2}-1\right)}{\sqrt{\frac{a b}{a g+b g+1}}}, \\
W_{4}= & -\frac{a\left(a^{2} g^{2}-1\right)\left(b^{2}-y^{2}\right)\left(b^{2}-z^{2}\right)}{\sqrt{\frac{a b}{a g+b g+1}}} .
\end{aligned}
$$

Note that we have added a pure gauge term to the 1 -form $\alpha_{6} d t$, where $\alpha_{6}=-1$.

Open Access. This article is distributed under the terms of the Creative Commons Attribution License (CC-BY 4.0), which permits any use, distribution and reproduction in any medium, provided the original author(s) and source are credited.

\section{References}

[1] J.M. Maldacena, The Large $N$ limit of superconformal field theories and supergravity, Int. J. Theor. Phys. 38 (1999) 1113 [hep-th/9711200] [INSPIRE].

[2] J. Kinney, J.M. Maldacena, S. Minwalla and S. Raju, An Index for 4 dimensional super conformal theories, Commun. Math. Phys. 275 (2007) 209 [hep-th/0510251] [INSPIRE].

[3] A. Cabo-Bizet, D. Cassani, D. Martelli and S. Murthy, Microscopic origin of the Bekenstein-Hawking entropy of supersymmetric AdS $S_{5}$ black holes, JHEP 10 (2019) 062 [arXiv: 1810.11442] [INSPIRE].

[4] S. Choi, J. Kim, S. Kim and J. Nahmgoong, Large AdS black holes from QFT, arXiv: 1810.12067 [INSPIRE].

[5] F. Benini and P. Milan, Black Holes in $4 D \mathcal{N}=4$ Super-Yang-Mills Field Theory, Phys. Rev. X 10 (2020) 021037 [arXiv: 1812.09613] [INSPIRE].

[6] S. Choi and S. Kim, Large AdS $S_{6}$ black holes from $C F T_{5}$, arXiv:1904.01164 [INSPIRE].

[7] G. Kántor, C. Papageorgakis and P. Richmond, AdS $S_{7}$ black-hole entropy and $5 D \mathcal{N}=2$ Yang-Mills, JHEP 01 (2020) 017 [arXiv: 1907.02923] [INSPIRE].

[8] J. Nahmgoong, 6d superconformal Cardy formulas, arXiv:1907.12582 [INSPIRE].

[9] S. Choi, C. Hwang and S. Kim, Quantum vortices, M2-branes and black holes, arXiv: 1908.02470 [INSPIRE].

[10] J. Nian and L.A. Pando Zayas, Microscopic entropy of rotating electrically charged $A d S_{4}$ black holes from field theory localization, JHEP 03 (2020) 081 [arXiv: 1909. 07943] [INSPIRE]. 
[11] N. Bobev and P.M. Crichigno, Universal spinning black holes and theories of class $\mathcal{R}$, JHEP 12 (2019) 054 [arXiv: 1909.05873] [INSPIRE].

[12] F. Benini, D. Gang and L.A. Pando Zayas, Rotating Black Hole Entropy from M5 Branes, JHEP 03 (2020) 057 [arXiv:1909.11612] [INSPIRE].

[13] S.M. Hosseini, K. Hristov and A. Zaffaroni, Microstates of rotating AdS $S_{5}$ strings, JHEP 11 (2019) 090 [arXiv: 1909.08000] [INSPIRE].

[14] P.M. Crichigno and D. Jain, The 5d Superconformal Index at Large $N$ and Black Holes, JHEP 09 (2020) 124 [arXiv:2005.00550] [INSPIRE].

[15] F. Benini, K. Hristov and A. Zaffaroni, Black hole microstates in AdS $S_{4}$ from supersymmetric localization, JHEP 05 (2016) 054 [arXiv:1511.04085] [INSPIRE].

[16] A. Zaffaroni, Lectures on AdS Black Holes, Holography and Localization, 2, 2019, DOI [arXiv: 1902.07176] [INSPIRE].

[17] D.E. Berenstein, J.M. Maldacena and H.S. Nastase, Strings in flat space and pp waves from $N=4$ superYang-Mills, JHEP 04 (2002) 013 [hep-th/0202021] [INSPIRE].

[18] M. Guica, T. Hartman, W. Song and A. Strominger, The Kerr/CFT Correspondence, Phys. Rev. D 80 (2009) 124008 [arXiv:0809.4266] [INSPIRE].

[19] H. Lü, J. Mei and C.N. Pope, Kerr/CFT Correspondence in Diverse Dimensions, JHEP 04 (2009) 054 [arXiv:0811.2225] [INSPIRE].

[20] D.D.K. Chow, M. Cvetič, H. Lü and C.N. Pope, Extremal Black Hole/CFT Correspondence in (Gauged) Supergravities, Phys. Rev. D 79 (2009) 084018 [arXiv:0812.2918] [InSPIRE].

[21] J.M. Bardeen and G.T. Horowitz, The Extreme Kerr throat geometry: A Vacuum analog of $A d S_{2} \times S^{2}$, Phys. Rev. D 60 (1999) 104030 [hep-th/9905099] [InSPIRE].

[22] A. Strominger, Black hole entropy from near horizon microstates, JHEP 02 (1998) 009 [hep-th/9712251] [INSPIRE].

[23] A. Strominger and C. Vafa, Microscopic origin of the Bekenstein-Hawking entropy, Phys. Lett. B 379 (1996) 99 [hep-th/9601029] [InSPIRE].

[24] K. Goldstein, V. Jejjala, Y. Lei, S. van Leuven and W. Li, Probing the EVH limit of supersymmetric AdS black holes, JHEP 02 (2020) 154 [arXiv:1910.14293] [InSPIRE].

[25] M.M. Sheikh-Jabbari and H. Yavartanoo, EVH Black Holes, AdS3 Throats and EVH/CFT Proposal, JHEP 10 (2011) 013 [arXiv:1107.5705] [InSPIRE].

[26] J. de Boer, M. Johnstone, M.M. Sheikh-Jabbari and J. Simon, Emergent IR Dual $2 d$ CFTs in Charged AdS5 Black Holes, Phys. Rev. D 85 (2012) 084039 [arXiv:1112.4664] [INSPIRE].

[27] M. Johnstone, M.M. Sheikh-Jabbari, J. Simon and H. Yavartanoo, Near-Extremal Vanishing Horizon AdS5 Black Holes and Their CFT Duals, JHEP 04 (2013) 045 [arXiv:1301.3387] [INSPIRE].

[28] S. Sadeghian, M.M. Sheikh-Jabbari, M.H. Vahidinia and H. Yavartanoo, Near Horizon Structure of Extremal Vanishing Horizon Black Holes, Nucl. Phys. B 900 (2015) 222 [arXiv: 1504.03607] [INSPIRE].

[29] Z.-W. Chong, M. Cvetič, H. Lü and C.N. Pope, General non-extremal rotating black holes in minimal five-dimensional gauged supergravity, Phys. Rev. Lett. 95 (2005) 161301 [hep-th/0506029] [INSPIRE]. 
[30] J.B. Gutowski and H.S. Reall, Supersymmetric AdS $S_{5}$ black holes, JHEP 02 (2004) 006 [hep-th/0401042] [INSPIRE].

[31] M. Honda, Quantum Black Hole Entropy from 4d Supersymmetric Cardy formula, Phys. Rev. D 100 (2019) 026008 [arXiv: 1901.08091] [INSPIRE].

[32] A. Arabi Ardehali, Cardy-like asymptotics of the $4 d \mathcal{N}=4$ index and AdS $S_{5}$ blackholes, JHEP 06 (2019) 134 [arXiv: 1902.06619] [INSPIRE].

[33] J. Kim, S. Kim and J. Song, $A 4 d N=1$ Cardy Formula, arXiv:1904.03455 [inSPIRE].

[34] A. Cabo-Bizet, D. Cassani, D. Martelli and S. Murthy, The asymptotic growth of states of the $4 d \mathcal{N}=1$ superconformal index, JHEP 08 (2019) 120 [arXiv:1904.05865] [INSPIRE].

[35] A. Amariti, I. Garozzo and G. Lo Monaco, Entropy function from toric geometry, arXiv: 1904.10009 [INSPIRE].

[36] A. González Lezcano and L.A. Pando Zayas, Microstate counting via Bethe Ansätze in the 4d $\mathcal{N}=1$ superconformal index, JHEP 03 (2020) 088 [arXiv: 1907.12841] [INSPIRE].

[37] A. Lanir, A. Nedelin and O. Sela, Black hole entropy function for toric theories via Bethe Ansatz, JHEP 04 (2020) 091 [arXiv: 1908.01737] [INSPIRE].

[38] F. Larsen, J. Nian and Y. Zeng, AdS $S_{5}$ black hole entropy near the BPS limit, JHEP 06 (2020) 001 [arXiv: 1907.02505] [inSPIRE].

[39] A. Cabo-Bizet and S. Murthy, Supersymmetric phases of $4 d \mathcal{N}=4 S Y M$ at large N, JHEP 09 (2020) 184 [arXiv: 1909.09597] [InSPIRE].

[40] A. Arabi Ardehali, J. Hong and J.T. Liu, Asymptotic growth of the $4 d \mathcal{N}=4$ index and partially deconfined phases, JHEP 07 (2020) 073 [arXiv: 1912.04169] [INSPIRE].

[41] A. Zaffaroni, Progress on AdS Black Holes in String Theory, talk given at Strings 2019.

[42] I. Bredberg, C. Keeler, V. Lysov and A. Strominger, Cargese Lectures on the Kerr/CFT Correspondence, Nucl. Phys. B Proc. Suppl. 216 (2011) 194 [arXiv:1103.2355] [InSPIRE].

[43] S.M. Hosseini, K. Hristov and A. Zaffaroni, An extremization principle for the entropy of rotating BPS black holes in AdS $S_{5}$, JHEP 07 (2017) 106 [arXiv: 1705.05383] [INSPIRE].

[44] S.M. Hosseini, K. Hristov and A. Zaffaroni, Gluing gravitational blocks for AdS black holes, JHEP 12 (2019) 168 [arXiv:1909.10550] [INSPIRE].

[45] Z.-W. Chong, M. Cvetič, H. Lü and C.N. Pope, Charged rotating black holes in four-dimensional gauged and ungauged supergravities, Nucl. Phys. B 717 (2005) 246 [hep-th/0411045] [INSPIRE].

[46] M. Cvetič, G.W. Gibbons, H. Lü and C.N. Pope, Rotating black holes in gauged supergravities: Thermodynamics, supersymmetric limits, topological solitons and time machines, hep-th/0504080 [INSPIRE].

[47] D.D.K. Chow and G. Compère, Dyonic AdS black holes in maximal gauged supergravity, Phys. Rev. D 89 (2014) 065003 [arXiv:1311.1204] [INSPIRE].

[48] S. Choi, C. Hwang, S. Kim and J. Nahmgoong, Entropy Functions of BPS Black Holes in $A d S_{4}$ and $A d S_{6}$, J. Korean Phys. Soc. 76 (2020) 101 [arXiv: 1811.02158] [INSPIRE].

[49] D. Cassani and L. Papini, The BPS limit of rotating AdS black hole thermodynamics, JHEP 09 (2019) 079 [arXiv: 1906.10148] [INSPIRE]. 
[50] J. Nian and X. Zhang, Entanglement Entropy of ABJM Theory and Entropy of Topological Black Hole, JHEP 07 (2017) 096 [arXiv: 1705.01896] [INSPIRE].

[51] Z.-W. Chong, M. Cvetič, H. Lü and C.N. Pope, Non-extremal charged rotating black holes in seven-dimensional gauged supergravity, Phys. Lett. B 626 (2005) 215 [hep-th/0412094] [INSPIRE].

[52] D.D.K. Chow, Equal charge black holes and seven dimensional gauged supergravity, Class. Quant. Grav. 25 (2008) 175010 [arXiv:0711.1975] [INSPIRE].

[53] J. Nian, Localization of Supersymmetric Chern-Simons-Matter Theory on a Squashed $S^{3}$ with $\mathrm{SU}(2) \times U(1)$ Isometry, JHEP 07 (2014) 126 [arXiv:1309.3266] [INSPIRE].

[54] S.M. Hosseini, K. Hristov and A. Zaffaroni, A note on the entropy of rotating BPS AdS $\times$ × $S^{4}$ black holes, JHEP 05 (2018) 121 [arXiv:1803.07568] [INSPIRE].

[55] D.D.K. Chow, Charged rotating black holes in six-dimensional gauged supergravity, Class. Quant. Grav. 27 (2010) 065004 [arXiv: 0808. 2728] [InSPIRE].

[56] J. Brown and M. Henneaux, Central Charges in the Canonical Realization of Asymptotic Symmetries: An Example from Three-Dimensional Gravity, Commun. Math. Phys. 104 (1986) 207 [INSPIRE].

[57] S. Carlip, Black hole entropy from conformal field theory in any dimension, Phys. Rev. Lett. 82 (1999) 2828 [hep-th/9812013] [INSPIRE].

[58] J. Nian and L.A. Pando Zayas, Toward an effective $C F T_{2}$ from $\mathcal{N}=4$ super Yang-Mills and aspects of Hawking radiation, JHEP 07 (2020) 120 [arXiv:2003.02770] [INSPIRE].

[59] M. David and J. Nian, Universal Entropy and Hawking Radiation of Near-Extremal AdS 4 Black Holes, arXiv:2009.12370 [INSPIRE].

[60] T. Azeyanagi, G. Compere, N. Ogawa, Y. Tachikawa and S. Terashima, Higher-Derivative Corrections to the Asymptotic Virasoro Symmetry of $4 d$ Extremal Black Holes, Prog. Theor. Phys. 122 (2009) 355 [arXiv: 0903.4176] [INSPIRE].

[61] N. Bobev, A.M. Charles, K. Hristov and V. Reys, The Unreasonable Effectiveness of Higher-Derivative Supergravity in AdS $S_{4}$ Holography, Phys. Rev. Lett. 125 (2020) 131601 [arXiv: 2006.09390] [INSPIRE].

[62] J.K. Ghosh and L.A. Pando Zayas, Comments on Sen's Classical Entropy Function for Static and Rotating $\mathrm{AdS}_{4}$ Black Holes, arXiv:2009.11147 [INSPIRE].

[63] J.F. Melo and J.E. Santos, Stringy corrections to the entropy of electrically charged supersymmetric black holes with $\mathrm{AdS}_{5} \times S^{5}$ asymptotics, arXiv:2007.06582 [INSPIRE].

[64] L. Di Pietro and Z. Komargodski, Cardy formulae for SUSY theories in $d=4$ and $d=6$, JHEP 12 (2014) 031 [arXiv: 1407.6061] [inSPIRE].

[65] A. Arabi Ardehali, High-temperature asymptotics of supersymmetric partition functions, JHEP 07 (2016) 025 [arXiv: 1512.03376] [INSPIRE].

[66] L. Di Pietro and M. Honda, Cardy Formula for 4d SUSY Theories and Localization, JHEP 04 (2017) 055 [arXiv: 1611.00380] [INSPIRE].

[67] C.-M. Chang, M. Fluder, Y.-H. Lin and Y. Wang, Proving the 6d Cardy Formula and Matching Global Gravitational Anomalies, arXiv:1910.10151 [INSPIRE].

[68] N. Banerjee, S. Dutta, S. Jain, R. Loganayagam and T. Sharma, Constraints on Anomalous Fluid in Arbitrary Dimensions, JHEP 03 (2013) 048 [arXiv:1206.6499] [INSPIRE]. 
[69] K. Jensen, R. Loganayagam and A. Yarom, Chern-Simons terms from thermal circles and anomalies, JHEP 05 (2014) 110 [arXiv:1311.2935] [INSPIRE].

[70] A. Castro, F. Larsen and I. Papadimitriou, $5 D$ rotating black holes and the $n A d S_{2} / n C F T_{1}$ correspondence, JHEP 10 (2018) 042 [arXiv: 1807.06988] [INSPIRE].

[71] U. Moitra, S.K. Sake, S.P. Trivedi and V. Vishal, Jackiw-Teitelboim Gravity and Rotating Black Holes, JHEP 11 (2019) 047 [arXiv: 1905.10378] [INSPIRE]. 